\title{
Impedance of a ceramic break and its resonance structures
}

\author{
Yoshihiro Shobuda \\ JAEA, 2-4 Shirakata Shirane, Tokaimura, Nakagun, Ibaraki 319-1195, Japan \\ Yong Ho Chin and Koji Takata \\ KEK, High Energy Accelerator Research Organization, 1-1 Oho, Tsukuba, Ibaraki 305-0801, Japan
}

(Received 16 January 2014; published 29 September 2014)

\begin{abstract}
A new theory is developed to evaluate longitudinal and transverse impedances of any size of ceramic break that is sandwiched between metal chambers. The theory has been numerically compared with the codes ABCI and CST STUDIO. Excellent agreements are obtained with both codes, in particular with ABCI. The theory successfully reproduces resonance structures of the impedance due to trapped modes inside the ceramic break, which are enhanced by the difference of the dielectric constants between the ceramic and the surrounding space. Moreover, the theory can evaluate the impedance of the ceramic break with titanium nitride coating. We discuss several characteristics of the impedances, especially the difference between the impedances of the ceramic break covered with and without a conductive wall on its outer surface.
\end{abstract}

DOI: 10.1103/PhysRevSTAB.17.091001

PACS numbers: 29.27.-a, 41.20.-q

\section{INTRODUCTION}

A short ceramic ring that is sandwiched by metal chambers is called a ceramic break. Such ceramic breaks are often inserted between chambers near bending magnets in proton synchrotrons, to mitigate the eddy current effects on the chambers excited by the outside time-varying magnetic fields [1]. The inner surface of the ceramic is sometimes coated with titanium nitride (TiN) to suppress the secondary emission of electrons, which is caused by the collisions between a part of a proton beam and the chamber surface. The TiN coating is beneficial to prevent the buildup of the electron cloud from destabilizing the beams [2-4]. On the other hand, these ceramic breaks are sources of the beam impedance $[5,6]$. The precise estimation of impedance is an important step toward realization of high intensity beams in proton synchrotrons [7].

The impedance of the ceramic break can be calculated in principle using three-dimensional simulation codes such as CST Studio [8] or 2.5-dimensional codes such as ABCI (for an axis-symmetrical structure) [9]. However, no comprehensive theory exists on this matter up to date. The lack of such a theory makes it difficult to understand the physical nature of the ceramic break impedance and to evaluate a degree of accuracy of numerical calculations, particularly, when agreements between different codes are not too great. As will be seen, the ceramic break impedances reveal resonant structures due to trapped modes inside the

Published by the American Physical Society under the terms of the Creative Commons Attribution 3.0 License. Further distribution of this work must maintain attribution to the author(s) and the published article's title, journal citation, and DOI. ceramic, and the theoretical approach provides an insight to the physical nature of these resonances.

From a practical point of view, simulation studies often encounter limitations in the estimate of the resistive wall impedances of conductive materials, because the mesh sizes need to be sufficiently smaller than the dimension of the materials or the skin depth. In particular, it is almost impossible to numerically estimate the impedances of a ceramic break with thin TiN coating.

Prior to the present paper, the authors have examined the impedance of a gap in slightly different configurations. The first paper is for a pure short gap (the gap is left empty in vacuum) [10]. The second case assumes that a short gap is filled with a thin resistive ring [11]. In both cases, the gap is sandwiched with thin metal chambers on both sides. In the present paper, we deal with a gap filled with a dielectric material. The theoretical framework is generalized at the same time so that the theory can now handle the ceramic break of any size, and is not limited by its thickness or length.

In Sec. II, we derive general formulas of the longitudinal and transverse impedances of a ceramic break. In Sec. III, we will conduct accuracy tests of the theory by numerical comparisons with the simulation codes ABCI and CST Studio. The characteristics of resonance structures appearing in the ceramic breaks are investigated. In Sec. IV, we evaluate the impedances of ceramic breaks with TiN coating by using the present theory. The study generalizes the previous results for a short gap filled with a thin resistive material. We discuss the difference between the impedances of the ceramic breaks covered with and without conductive walls on their outer surface. In Sec. V, the Lorentz- $\gamma$ dependence of the impedances is investigated. The paper is summarized in Sec. VI. 


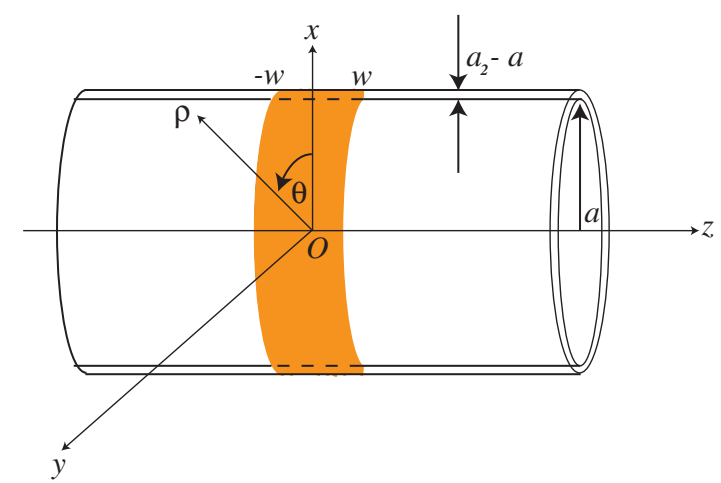

FIG. 1. A schematic picture of ceramic (orange object), which is sandwiched by perfectly conductive metal chambers. The inner and the outer radii and the length of the ceramic are $a, a_{2}$ and $g(=2 w)$, respectively. Since the cylindrical coordinates $(\rho, \theta, z)$ are used, the radial, the azimuthal and the longitudinal components of electric and magnetic fields are represented as $E_{\rho}, H_{\rho}$, $E_{\theta}, H_{\theta}$ and $E_{z}, H_{z}$, respectively.

\section{FORMULAS FOR IMPEDANCES OF A CERAMIC BREAK}

\section{A. Longitudinal impedances}

Let us start with deriving electromagnetic fields generated by the interaction between a beam and an axisymmetric ceramic break. As shown in Fig. 1, the cylindrical coordinates $(\rho, \theta, z)$ are used. The outer and the inner radii of the ceramic (and those of the chambers of the both sides) are given by $a_{2}$ and $a$, respectively. The ceramic is located in the region $-w<z<w$ (namely, the length $g$ of the ceramic is equal to $2 w$ ). In order to obtain formal solutions of the fields, the field matching technique is applied to this system [12]. Unlike the previous studies [10,11], the ceramic ring can be thick, and the length $g$ of the ring can be arbitrary.

It is assumed that the beam has a cylindrically uniform density with radius $\sigma$ and its total charge is $1 \mathrm{C} / \mathrm{m}$. Namely, its current density is given by

$$
j_{z}=\beta c[1-\Theta(\rho-\sigma)] e^{-j k z+j \omega t} /\left(\pi \sigma^{2}\right),
$$

where $\Theta(x)$ is the step function, $k=\omega / \beta c, \beta=v / c, v$ is the velocity of the beam, $c$ is the velocity of light, $\omega=2 \pi f$ and $f$ is the frequency. The formal solutions of the fields at the angular frequency $\omega$ inside the chamber are given by

$$
\begin{aligned}
E_{z}= & \frac{j c Z_{0}}{\pi \sigma^{2} \gamma}\left(\frac{1}{\bar{k}}-\sigma I_{0}(\bar{k} \rho) K_{1}(\bar{k} \sigma)-\frac{\sigma I_{0}(\bar{k} \rho) I_{1}(\bar{k} \sigma) K_{0}(\bar{k} a)}{I_{0}(\bar{k} a)}\right) e^{-j k z} \\
& +\int_{-\infty}^{\infty} d h e^{-j h z} \frac{J_{0}(\Lambda \rho)}{J_{0}(\Lambda a)}\left[\frac{V_{1}}{2 \pi} \frac{\sin h w}{h w}+\sum_{m=1}^{\infty} \frac{V_{1}^{(m)}\left[(-1)^{m} e^{j h w}-e^{-j h w}\right] h}{j 2 \pi w\left(h^{2}-\frac{m^{2} \pi^{2}}{4 w^{2}}\right)}\right], \\
H_{\theta}= & \frac{\beta c}{\pi \sigma}\left(K_{1}(\bar{k} \sigma)+\frac{I_{1}(\bar{k} \sigma) K_{0}(\bar{k} a)}{I_{0}(\bar{k} a)}\right) I_{1}(\bar{k} \rho) e^{-j k z} \\
+ & \frac{j k \beta}{Z_{0}} \int_{-\infty}^{\infty} d h e^{-j h z} \frac{J_{1}(\Lambda \rho)}{\Lambda J_{0}(\Lambda a)}\left[\frac{V_{1}}{2 \pi} \frac{\sin h w}{h w}+\sum_{m=1}^{\infty} \frac{V_{1}^{(m)}\left[(-1)^{m} e^{j h w}-e^{-j h w}\right] h}{j 2 \pi w\left(h^{2}-\frac{m^{2} \pi^{2}}{4 w^{2}}\right)}\right],
\end{aligned}
$$

for $\rho<\sigma$, and

$$
\begin{aligned}
E_{z}= & \frac{j c Z_{0}}{\pi \sigma \gamma} I_{1}(\bar{k} \sigma)\left(K_{0}(\bar{k} \rho)-\frac{I_{0}(\bar{k} \rho) K_{0}(\bar{k} a)}{I_{0}(\bar{k} a)}\right) e^{-j k z} \\
& +\int_{-\infty}^{\infty} d h e^{-j h z} \frac{J_{0}(\Lambda \rho)}{J_{0}(\Lambda a)}\left[\frac{V_{1}}{2 \pi} \frac{\sin h w}{h w}+\sum_{m=1}^{\infty} \frac{V_{1}^{(m)}\left[(-1)^{m} e^{j h w}-e^{-j h w}\right] h}{j 2 \pi w\left(h^{2}-\frac{m^{2} \pi^{2}}{4 w^{2}}\right)}\right], \\
H_{\theta}= & \frac{\beta c}{\pi \sigma} I_{1}(\bar{k} \sigma)\left(K_{1}(\bar{k} \rho)+\frac{K_{0}(\bar{k} a) I_{1}(\bar{k} \rho)}{I_{0}(\bar{k} a)}\right) e^{-j k z} \\
& +\frac{j k \beta}{Z_{0}} \int_{-\infty}^{\infty} d h e^{-j h z} \frac{J_{1}(\Lambda \rho)}{\Lambda J_{0}(\Lambda a)}\left[\frac{V_{1}}{2 \pi} \frac{\sin h w}{h w}+\sum_{m=1}^{\infty} \frac{V_{1}^{(m)}\left[(-1)^{m} e^{j h w}-e^{-j h w}\right] h}{j 2 \pi w\left(h^{2}-\frac{m^{2} \pi^{2}}{4 w^{2}}\right)}\right],
\end{aligned}
$$


for $\rho>\sigma$, where $\Lambda=\sqrt{k^{2} \beta^{2}-h^{2}}, Z_{0}(=120 \pi \Omega)$ is the impedance of free space, $J_{n}(z)$ is the Bessel function, $I_{n}(z)$ and $K_{n}(z)$ are the modified Bessel functions [13], $\gamma$ is Lorentz- $\gamma, \bar{k}=k / \gamma$ and $V_{1}$ is the voltage on the inner surface of the ceramic at $\rho=a$ and $V_{1}^{(m)}$ are expansion coefficients.

The average value of $E_{z}$ [expressed by Eq. (2)] over $\rho$ gives the longitudinal impedance $Z_{L}$. It can be expressed as

$$
\begin{gathered}
Z_{L}=-\frac{j Z_{0}}{k \beta \pi \sigma^{2}}\left(1-\frac{2 K_{0}(\bar{k} a) I_{1}^{2}(\bar{k} \sigma)}{I_{0}(\bar{k} a)}-2 I_{1}(\bar{k} \sigma) K_{1}(\bar{k} \sigma)\right) \mathcal{L}+Z_{\text {break }, L} \\
Z_{\text {break }, L}=-\frac{2 I_{1}(\bar{k} \sigma)}{c \beta \sigma \bar{k} I_{0}(\bar{k} a)}\left[V_{1} \frac{\sin k w}{k w}+\sum_{m=1}^{\infty} \frac{V_{1}^{(m)} k\left[(-1)^{m} e^{j k w}-e^{-j k w}\right]}{j w\left(k^{2}-\frac{m^{2} \pi^{2}}{4 w^{2}}\right)}\right],
\end{gathered}
$$

where $\mathcal{L}$ is the length of the beam pipe. The first term and the second one $Z_{\text {break }, L}$ in Eq. (6) represent the nonrelativistic space charge impedance [10] and the coupling impedance of the ceramic break, respectively. The expansion coefficients $V_{1}$ and $V_{1}^{(m)}$ up to any order of $m$ are obtained by solving Eqs. (A20)-(A23) in Appendix A 1. The positive integer $m$ denotes the number of half wavelength in the ceramic. This mode plays an important role to explain the resonance structure of the impedance. The detail will be discussed in Sec. III C.

When the thickness $a_{2}-a$ and the length $g$ of the ceramic are sufficiently small, compared to the radial size

of the chamber $a$, a simple expression of longitudinal impedance is approximately obtained as

$$
\begin{aligned}
& Z_{\text {break }, L} \simeq-\frac{2 I_{1}(\bar{k} \sigma)}{c \beta \sigma \bar{k} I_{0}(\bar{k} a)}\left[V_{1} \frac{\sin k w}{k w}-\frac{2 V_{1}^{(1)} k \cos k w}{j w\left(k^{2}-\frac{\pi^{2}}{4 w^{2}}\right)}\right], \\
& V_{1}=\frac{-\frac{j k w \beta\langle Y(z)\rangle}{\pi Z_{0}} C_{0}^{(0)}\langle\alpha(z)\rangle-K_{0}^{(0)}\langle\alpha(z)\rangle}{\frac{I_{0}^{(0)}}{2 w}+\frac{K_{0}^{(0)} j k \beta\langle J(z)\rangle}{2 \pi Z_{0}}+\left(A_{0}^{(0)}+\frac{C_{0}^{(0)} j k \beta w\langle J(z)\rangle}{\pi Z_{0}}\right) \frac{j k \beta\langle Y(z)\rangle}{2 \pi Z_{0}}},
\end{aligned}
$$

where $\langle\alpha(z)\rangle,\langle\langle\alpha(z)\rangle\rangle_{m},\langle J(z)\rangle,\langle\langle J(z)\rangle\rangle_{m},\left\langle\left\langle J^{(n)}(z)\right\rangle\right\rangle_{m}$, $\langle Y(z)\rangle,\langle\langle Y(z)\rangle\rangle_{m},\left\langle\left\langle Y^{(n)}(z)\right\rangle_{m}\right.$ are given in Appendix B. The coefficients $A_{0}^{(0)}, C_{0}^{(0)}, I_{0}^{(0)}, K_{0}^{(0)}, A_{0}^{(m)}, C_{0}^{(m)}, I_{0}^{(m)}$ and $K_{0}^{(m)}$ with positive integer $m$ represent the transfer rates of the fields on the inner surface $(\rho=a)$ to the outer surface $\left(\rho=a_{2}\right)$ of the ceramic, which are determined by the solutions of Maxwell equations in the ceramic. The concrete expressions of transfer coefficients are given in Appendix C 1.

In Secs. III and IV, explicit calculations of impedances are done for several different sizes of ceramic, and accuracy tests are carried out between the theoretical and numerical simulation results.

\section{B. Transverse impedance}

Following the derivation of longitudinal impedance, let us consider the situation where a beam is traveling in the chamber with the charge distribution of the azimuthal dependence as $j_{z}=q \beta c \delta\left(\rho-r_{b}\right) \cos \theta e^{-j k z} / \pi r_{b}$. At first, let us describe the fields inside the chamber as

for $\rho<r_{b}$,

$$
\begin{aligned}
E_{z}= & i_{1}\left[\frac{j k}{\gamma^{2}} \frac{c Z_{0}}{\pi}\left(K_{1}\left(\bar{k} r_{b}\right)-K_{1}(\bar{k} a) \frac{I_{1}\left(\bar{k} r_{b}\right)}{I_{1}(\bar{k} a)}\right) I_{1}(\bar{k} \rho) \frac{e^{-j k z}}{r_{b}}\right. \\
& \left.+\int_{-\infty}^{\infty} d h e^{-j h z}\left(\tilde{\mathcal{V}}_{2} \frac{\sin h w}{h w}+\sum_{m=1}^{\infty} \tilde{\mathcal{V}}_{2}^{(m)} \frac{h\left[(-1)^{m} e^{j h w}-e^{-j h w}\right]}{j 2 w\left(h^{2}-\frac{m^{2} \pi^{2}}{4 w^{2}}\right)}\right) \frac{J_{1}(\Lambda \rho)}{J_{1}(\Lambda a)}\right] \cos \theta,
\end{aligned}
$$




$$
\begin{aligned}
& E_{z}=i_{1}\left[\frac{j k}{\gamma^{2}} \frac{c Z_{0}}{\pi}\left(K_{1}(\bar{k} \rho)-\frac{I_{1}(\bar{k} \rho)}{I_{1}(\bar{k} a)} K_{1}(\bar{k} a)\right) I_{1}\left(\bar{k} r_{b}\right) \frac{e^{-j k z}}{r_{b}}\right. \\
& \left.+\int_{-\infty}^{\infty} d h e^{-j h z}\left(\tilde{\mathcal{V}}_{2} \frac{\sin h w}{h w}+\sum_{m=1}^{\infty} \tilde{\mathcal{V}}_{2}^{(m)} \frac{h\left[(-1)^{m} e^{j h w}-e^{-j h w}\right]}{j 2 w\left(h^{2}-\frac{m^{2} \pi^{2}}{4 w^{2}}\right)}\right) \frac{J_{1}(\Lambda \rho)}{J_{1}(\Lambda a)}\right] \cos \theta, \\
& H_{\theta}=i_{1}\left\{c \beta \frac{\bar{k}}{2 \pi}\left[K_{0}(\bar{k} \rho)+K_{2}(\bar{k} \rho)\right] I_{1}\left(\bar{k} r_{b}\right) \frac{e^{-j k z}}{r_{b}}+c \beta \frac{\bar{k}}{\pi I_{1}(\bar{k} a)} K_{1}(\bar{k} a) I_{1}^{\prime}(\bar{k} \rho) I_{1}\left(\bar{k} r_{b}\right) \frac{e^{-j k z}}{r_{b}}\right. \\
& -\int_{-\infty}^{\infty} d h e^{-j h z}\left[\sum_{m=1}^{\infty} \tilde{\mathcal{V}}_{1}^{(m)} \frac{m \pi\left[(-1)^{m} e^{j h w}-e^{-j h w}\right]}{4 w^{2}\left(h^{2}-\frac{m^{2} \pi^{2}}{4 w^{2}}\right)} \frac{h}{Z_{0} \Lambda} \frac{J_{1}(\Lambda \rho)}{k \beta \rho J_{1}{ }^{\prime}(\Lambda a)}\right. \\
& \left.\left.+\left(\tilde{\mathcal{V}}_{2} \frac{\sin h w}{h w}+\sum_{m=1}^{\infty} \tilde{\mathcal{V}}_{2}^{(m)} \frac{h\left[(-1)^{m} e^{j h w}-e^{-j h w}\right]}{j 2 w\left(h^{2}-\frac{m^{2} \pi^{2}}{4 w^{2}}\right)}\right) \frac{j h}{Z_{0} \Lambda}\left(\frac{k \beta J_{1}^{\prime}(\Lambda \rho)}{h J_{1}(\Lambda a)}-\frac{h J_{1}(\Lambda \rho)}{\rho k \beta a \Lambda^{2} J_{1}^{\prime}(\Lambda a)}\right)\right]\right\} \cos \theta, \\
& H_{z}=i_{1} \int_{-\infty}^{\infty} d h e^{-j h z}\left[\sum_{m=1}^{\infty} \tilde{\mathcal{V}}_{1}^{(m)} \frac{m \pi\left[(-1)^{m} e^{j h w}-e^{-j h w}\right]}{4 w^{2}\left(h^{2}-\frac{m^{2} \pi^{2}}{4 w^{2}}\right)}-\left(\tilde{\mathcal{V}}_{2} \frac{\sin h w}{h w}+\sum_{m=1}^{\infty} \tilde{\mathcal{V}}_{2}^{(m)} \frac{h\left[(-1)^{m} e^{j h w}-e^{-j h w}\right]}{j 2 w\left(h^{2}-\frac{m^{2} \pi^{2}}{4 w^{2}}\right)}\right) \frac{j h}{\Lambda^{2} a}\right] \\
& \times \frac{\Lambda}{j k \beta Z_{0}} \frac{J_{1}(\Lambda \rho)}{J_{1}^{\prime}(\Lambda a)} \sin \theta \\
& E_{\theta}=i_{1}\left[\frac{c Z_{0}}{\pi \rho}\left(K_{1}(\bar{k} \rho)-\frac{I_{1}(\bar{k} \rho)}{I_{1}(\bar{k} a)} K_{1}(\bar{k} a)\right) I_{1}\left(\bar{k} r_{b}\right) \frac{e^{-j k z}}{r_{b}}+\int_{-\infty}^{\infty} d h e^{-j h z} \sum_{m=1}^{\infty} \tilde{\mathcal{V}}_{1}^{(m)} \frac{m \pi\left[(-1)^{m} e^{j h w}-e^{-j h w}\right]}{4 w^{2}\left(h^{2}-\frac{m^{2} \pi^{2}}{4 w^{2}}\right)} \frac{J_{1}^{\prime}(\Lambda \rho)}{J_{1}^{\prime}(\Lambda a)}\right. \\
& \left.+\int_{-\infty}^{\infty} d h e^{-j h z} \frac{j h}{\Lambda^{2}}\left(\sum_{m=1}^{\infty} \tilde{\mathcal{V}}_{2} \frac{\sin h w}{h w}+\tilde{\mathcal{V}}_{2}^{(m)} \frac{h\left[(-1)^{m} e^{j h w}-e^{-j h w}\right]}{j 2 w\left(h^{2}-\frac{m^{2} \pi^{2}}{4 w^{2}}\right)}\right)\left(\frac{J_{1}(\Lambda \rho)}{\rho J_{1}(\Lambda a)}-\frac{J_{1}^{\prime}(\Lambda \rho)}{a J_{1}^{\prime}(\Lambda a)}\right)\right] \sin \theta,
\end{aligned}
$$

for $\rho>r_{b}$. Here, $i_{1}=q r_{b}$, the prime in $I_{n}(z)$ and $J_{n}(z)$ means the differential by their argument $z, \tilde{\mathcal{V}}_{2}$ is the voltage on the inner surface of the ceramic at $\rho=a$ and $\tilde{\mathcal{V}}_{1}^{(m)}$ and $\tilde{\mathcal{V}}_{2}^{(m)}$ are expansion coefficients.

Using the Panofsky-Wenzel theorem [5,14], we finally obtain the expression for the transverse impedance as

$$
\begin{gathered}
Z_{T}=\frac{R Z_{0} k}{j \gamma^{3} \beta r_{b}}\left(K_{1}\left(\bar{k} r_{b}\right)-K_{1}(\bar{k} a) \frac{I_{1}\left(\bar{k} r_{b}\right)}{I_{1}(\bar{k} a)}\right)+Z_{\text {break }, T}, \\
Z_{\text {break }, T}=-\frac{\pi}{c \beta \gamma I_{1}(\bar{k} a)}\left[\tilde{\mathcal{V}}_{2} \frac{\sin k w}{k w}+\sum_{m=1}^{\infty} \tilde{\mathcal{V}}_{2}^{(m)} \frac{k\left[(-1)^{m} e^{j k w}-e^{-j k w}\right]}{j 2 w\left(k^{2}-\frac{m^{2} \pi^{2}}{4 w^{2}}\right)}\right] .
\end{gathered}
$$

The first term and the second one $Z_{\mathrm{break}, T}$ of Eq. (17) represent the transverse space charge impedance for a nonrelativistic beam [10] and the transverse impedance of the ceramic break, respectively. By solving Eqs. (A66)-(A71) in Appendix A 2, the expansion coefficients $\tilde{\mathcal{V}}_{2}$ and $\tilde{\mathcal{V}}_{2}^{(m)}$ are obtained for any order of $m$. The precision of the theoretical results can be improved by increasing the correction order $m$ in Eq. (18).

In the lowest order approximation, the expression is simplified as

$$
Z_{\text {break }, T}=-\frac{\pi \tilde{\mathcal{V}}_{2}}{c \beta \gamma I_{1}(\bar{k} a)} \frac{\sin k w}{k w},
$$

where

$$
\tilde{\mathcal{V}}_{2}=\frac{\left[\frac{j k \beta\left\langle Y_{5}(z)-\frac{Y_{6}(z)}{k^{2} \beta^{2} a_{2}^{2}}\right\rangle}{Z_{0}} C_{1}^{(0)}+\frac{\pi K_{1}^{(0)}}{w}\right] \frac{c \beta I_{1}\left(\bar{k} r_{b}\right)}{r_{b} \pi a I_{1}(\bar{k} a)} \frac{\sin k w}{k w}}{-\left(\frac{A_{1}^{(0)} \pi}{w}-\frac{C_{1}^{(0)} j k \beta\left\langle Y_{2}(z)-\frac{Y_{3}(z)}{\left.k^{2} \beta^{2} a^{2}\right\rangle}\right.}{Z_{0}}\right) \frac{j k \beta\left\langle Y_{5}(z)-\frac{Y_{6}(z)}{\left.k^{2} \beta^{2} a_{2}^{2}\right\rangle}\right.}{Z_{0}}+\frac{\pi}{w}\left(-\frac{I_{1}^{(0)} \pi}{w}+\frac{K_{1}^{(0)} j k \beta\left\langle Y_{2}(z)-\frac{Y_{3}(z)}{\left.k^{2} \beta^{2} a^{2}\right\rangle}\right.}{Z_{0}}\right)},
$$


$\left\langle Y_{2}(z)-Y_{3}(z) / k^{2} \beta^{2} a^{2}\right\rangle,\left\langle Y_{5}(z)-Y_{6}(z) / k^{2} \beta^{2} a_{2}^{2}\right\rangle$ are given by Eqs. (D12) and (D14) in Appendix D, $A_{1}^{(0)}, C_{1}^{(0)}, I_{1}^{(0)}$ and $K_{1}^{(0)}$ are transfer coefficients for the transverse impedance, and the concrete expressions are shown in Appendix C 2.

\section{NUMERICAL COMPARISON BETWEEN THEORETICAL AND SIMULATION RESULTS}

In the last section, we obtain the theoretical expressions of impedances of ceramic break [Eqs. (7) and (18)]. Let us now compare the theoretical results and numerical simulation results for a relativistic beam.

For the theoretical analysis, the transfer coefficients $A_{0}^{(0)}, C_{0}^{(0)}, I_{0}^{(0)}, K_{0}^{(0)}, A_{0}^{(m)}, C_{0}^{(m)}, I_{0}^{(m)}$ and $K_{0}^{(m)}$ are given by Eqs. (C10)-(C17) in Appendix C 1 for the longitudinal impedance. The solutions of Eqs. (A20)-(A23) in Appendix A 1 with the transfer coefficients provide the expansion coefficients $V_{1}$ and $V_{1}^{(m)}$. The final theoretical results are obtained by substituting the obtained expansion coefficients into Eq. (7). The transfer coefficients for the transverse impedances $A_{1}^{(0)}, C_{1}^{(0)}, I_{1}^{(0)}, K_{1}^{(0)}, A_{1}^{(m)}, B_{1}^{(m)}$, $C_{1}^{(m)}, E_{1}^{(m)}, F_{1}^{(m)}, H_{1}^{(m)}, I_{1}^{(m)}, J_{1}^{(m)}, K_{1}^{(m)}, L_{1}^{(m)}, M_{1}^{(m)}, N_{1}^{(m)}$, $O_{1}^{(m)}$ and $P_{1}^{(m)}$ are given by Eqs. (C31)-(C34) and (C36)$(\mathrm{C} 51)$ in Appendix C2. The expansion coefficients $\tilde{\mathcal{V}}_{2}$ and $\tilde{\mathcal{V}}_{2}^{(m)}$ are obtained by solving Eqs. (A66)-(A71) in Appendix A 2 combining with Eqs. (C31)-(C34) and (C36)-(C51). The final theoretical results for the transverse impedance are provided by substituting the solutions $\tilde{\mathcal{V}}_{2}$ and $\tilde{\mathcal{V}}_{2}^{(m)}$ into Eq. (18).

The ABCI code does not have an option to simulate the open boundary condition far from the ceramic break in the radial direction. Instead, we put a large cavity outside the ceramic break, so large that the fields radiated from the ceramic break cannot come back to their origin after bouncing back at the cavity wall before the test particle at the very end of the wake potential coordinate passes the ceramic break. In other words, the radius of the outer cavity needs to be at least a half of the length of the wake potential to be calculated. The maximum radius of the cavity used in all the calculation is $20 \mathrm{~m}$ (the longitudinal length of the cavity is $40 \mathrm{~m}$ ). The mesh size is $0.625 \mathrm{~mm}$ in both the radial and the longitudinal directions. The root mean square (rms) bunch length used is $10 \mathrm{~mm}$, though the impedance does not depend on the choice of bunch length in the frequency range of interest.

On the other hand, CST Studio can simulate the open boundary condition [the option is "open (add space)"]. Inside the ceramic, we used a finer mesh than in other parts, typically $0.5 \mathrm{~mm}$. The rms bunch length used is $5 \mathrm{~mm}$. Again, the final impedance has no dependence on the choice of the bunch length.

\section{A. Vacuum gap case}

The first example is a vacuum gap $\left[\epsilon^{\prime}=1, \mu^{\prime}=1, \sigma_{c}\right.$ (conductivity) $=0]$. The inner and the outer radii of the chambers are $a=65 \mathrm{~mm}, a_{2}=70 \mathrm{~mm}$, respectively. The gaps length $g$ is $10 \mathrm{~mm}$. The calculation results for the longitudinal and the transverse impedances are shown by the left and the right parts of Fig. 2, respectively. The red, the black and the blue lines show the theoretical, ABCI's and CST's results, respectively. The solid and the dashed lines show the real and the imaginary parts of impedances, respectively. The existence of a large resonance at low
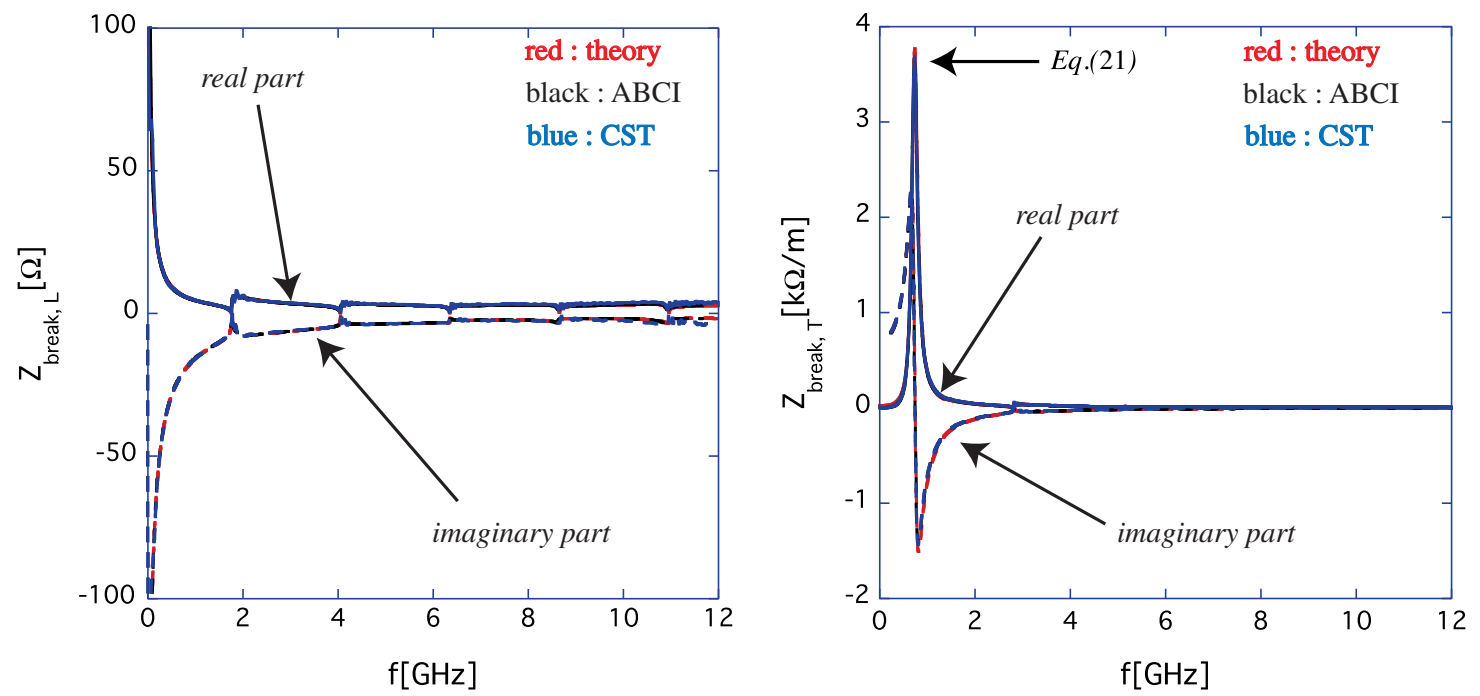

FIG. 2. Impedances of a vacuum gap ( $a=65 \mathrm{~mm}, a_{2}=70 \mathrm{~mm}$ and $g=10 \mathrm{~mm}$ ) calculated by the theory (red), the simulation codes ABCI (black) and CST Studio (blue). The left and the right figures show the longitudinal and the transverse impedances, respectively. The solid and the dashed lines show the real and the imaginary parts of the impedances, respectively. 

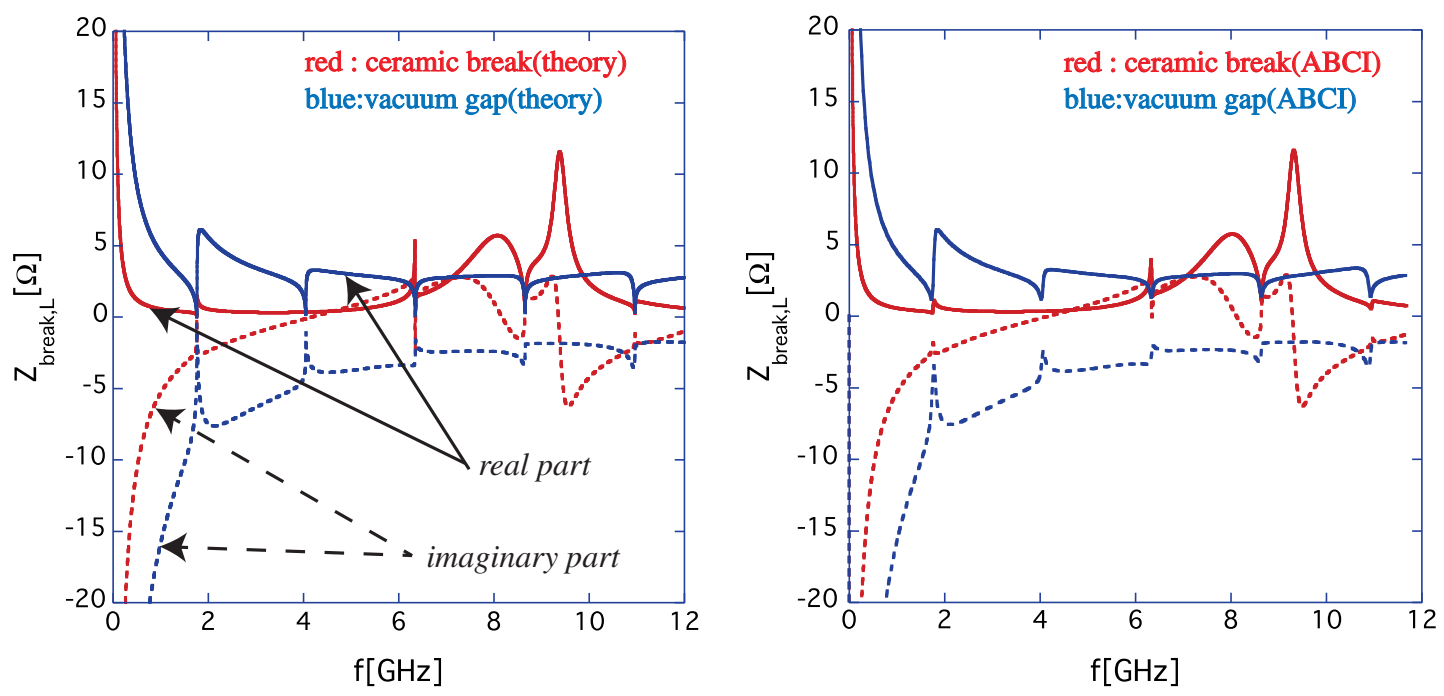

FIG. 3. Longitudinal impedance of the ceramic break $\left(\epsilon^{\prime}=11\right)$ (red) and that of the vacuum gap (blue). The left and the right parts show the theoretical result and the numerical one calculated by ABCI, respectively. The solid and the dashed lines show the real and the imaginary parts of impedances, respectively.

frequency in the transverse impedance was already reported in the previous paper [10]. The frequency is given by

$$
f \sim \frac{c}{2 \pi a}
$$

(i.e., the wavelength resonates along the circumference of the chamber $2 \pi a$ ).

In this paper, the theory is expanded from that in Ref. [10] to cope with a thick gap sandwiched by chambers with finite thickness. All three results are in good agreement, which indicates that the generalization was done successfully and can accurately describe the gap impedances.

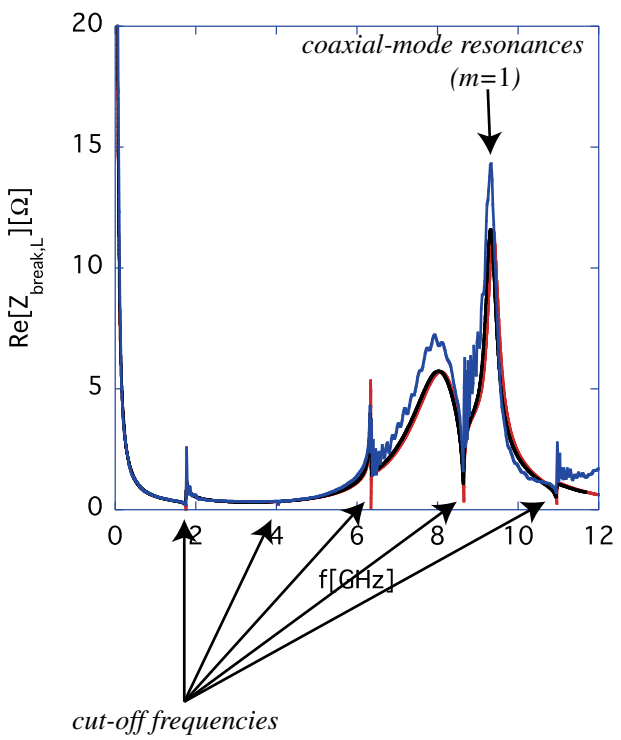

\section{B. Overview of longitudinal impedances of the vacuum gap and the ceramic break}

Let us take an overview of the longitudinal impedance of the ceramic break $\left(\epsilon^{\prime}=11\right)$ and that of the vacuum gap for the values of $a=65 \mathrm{~mm}, a_{2}=70 \mathrm{~mm}$ and $g=10 \mathrm{~mm}$ (this is standard size of the ceramic break in use at the Main Ring (MR) in Japan Accelerator Research Complex (J-PARC) [7]). The left and the right parts of Fig. 3 show the theoretical result and the numerical one calculated by $\mathrm{ABCI}$, respectively. The red and the blue lines show the impedances for the ceramic break and those for the vacuum gap, respectively. The solid and the dashed lines show the real and the imaginary parts of impedances, respectively.

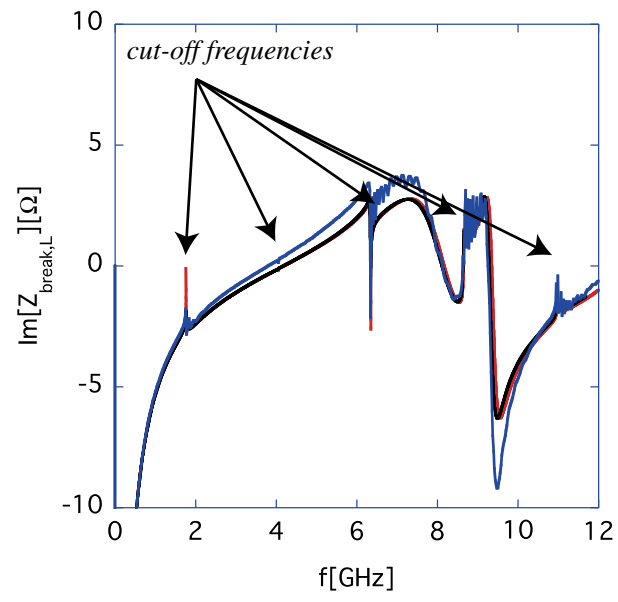

FIG. 4. Longitudinal impedances of the ceramic break ( $a=65 \mathrm{~mm}, a_{2}=70 \mathrm{~mm}, g=10 \mathrm{~mm}$ and $\left.\epsilon^{\prime}=11\right)$ calculated by the theory (red), the simulation codes ABCI (black) and CST Studio (blue). 

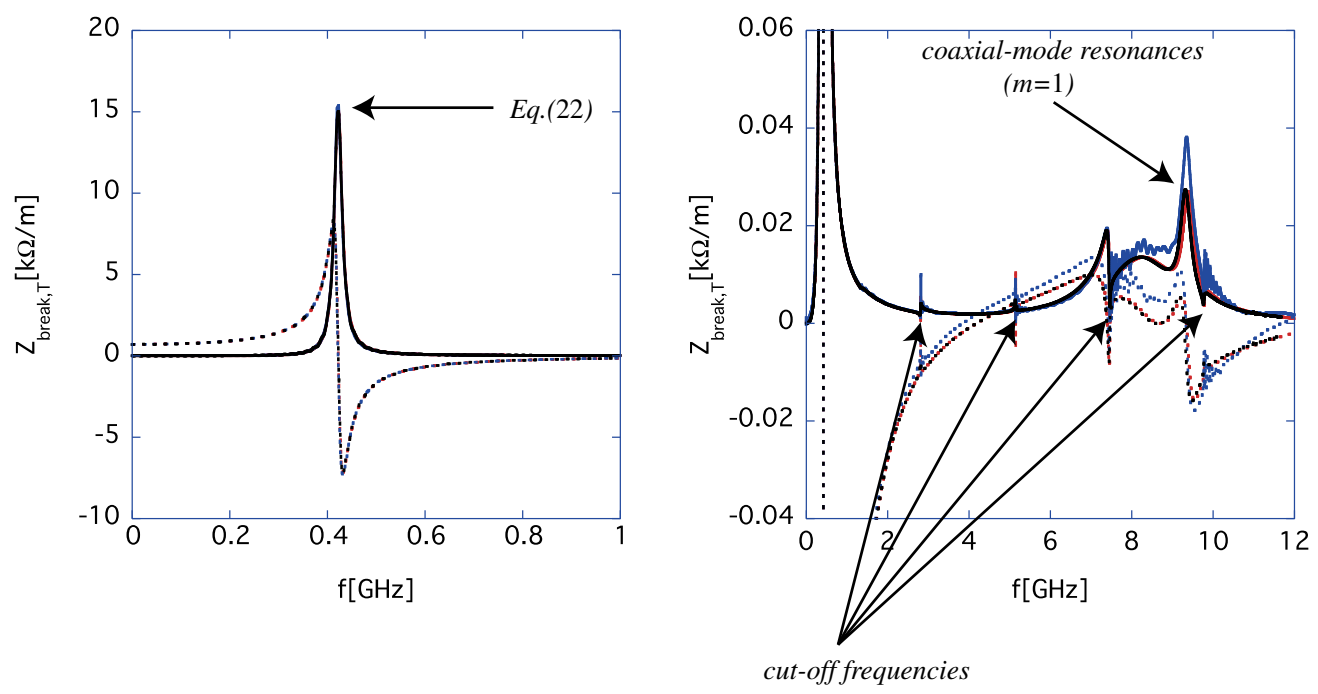

FIG. 5. Transverse impedances of the ceramic break ( $a=65 \mathrm{~mm}, a_{2}=70 \mathrm{~mm}, g=10 \mathrm{~mm}$ and $\left.\epsilon^{\prime}=11\right)$ calculated by the theory (red), the simulation codes ABCI (black) and CST Studio (blue). The solid and the dot lines show the real and the imaginary parts of the impedance, respectively. The left and the right figures show the results up to $1 \mathrm{GHz}$ frequency, and those up to $12 \mathrm{GHz}$, respectively.

The cutoff frequencies are identical for both the ceramic break and the vacuum gap. It can be clearly seen that another type of resonance structure is excited in the ceramic break at high frequency. Despite the existence of the resonances, the loss factor of the ceramic break is a few times smaller than that of the vacuum gap (the loss factors for the ceramic break and the vacuum gap are numerically found to be 22.34 and $73.44 \mathrm{mV} / \mathrm{pC}$, respectively). This is because the impedance decays more rapidly in the ceramic as a function of frequency due to the wavelength contraction effect. Thereby, the ceramic is in general beneficial to suppress the energy loss of the beam, in particular, for a long bunch.
The above observations show that the impedance of the ceramic break has different characteristics from that of the simple gap. In the following sections, let us investigate these new phenomena more closely.

\section{Impedances of ceramic break and their resonance structures}

Let us continue numerical comparisons for the same size of ceramic break ( $a=65 \mathrm{~mm}, a_{2}=70 \mathrm{~mm}$ and $g=10 \mathrm{~mm}$ ) and discuss the resonance structures. The longitudinal and the transverse impedances are shown in Figs. 4 and 5, respectively.
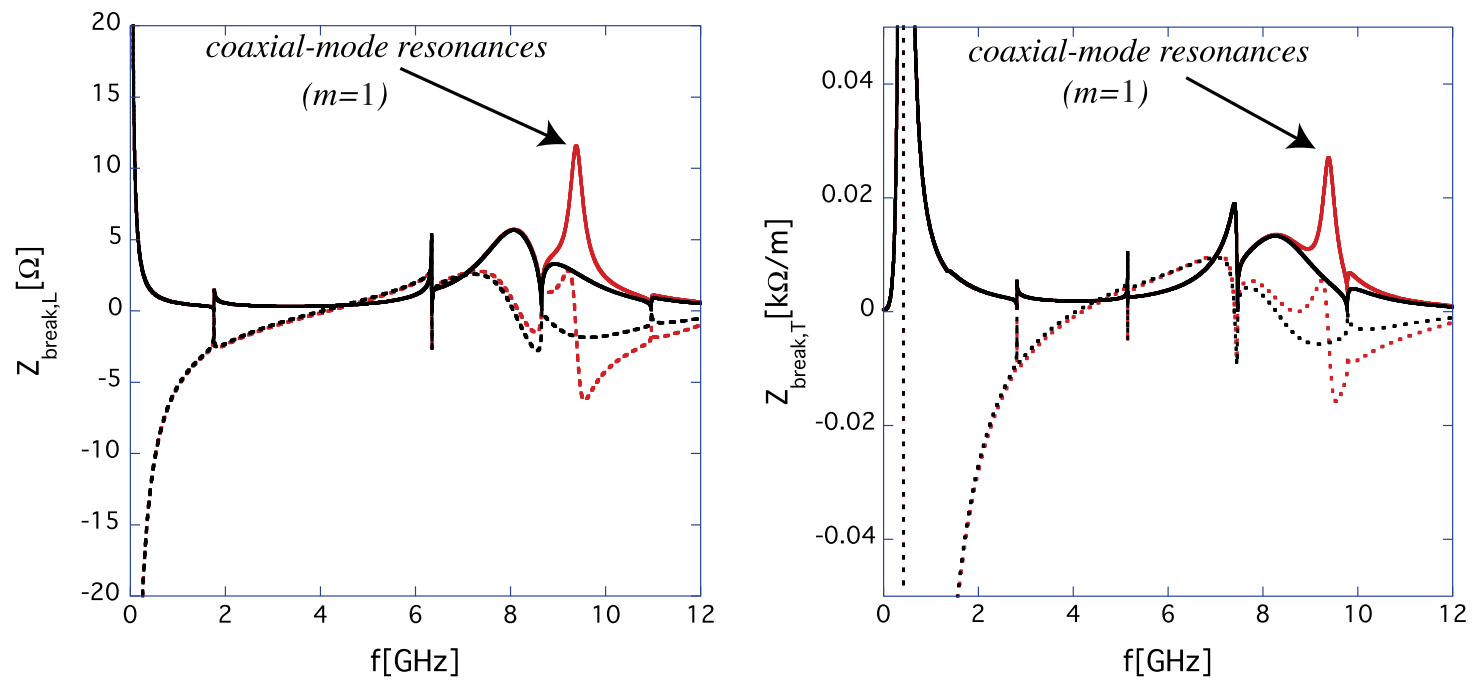

FIG. 6. Theoretical results of impedances of the ceramic break ( $a=65 \mathrm{~mm}, a_{2}=70 \mathrm{~mm}, g=10 \mathrm{~mm}$ and $\left.\epsilon^{\prime}=11\right)$. The red line shows the calculation results when the higher order term up to $m=2$ is included, while the black line shows the results when only the lowest order term is considered. 


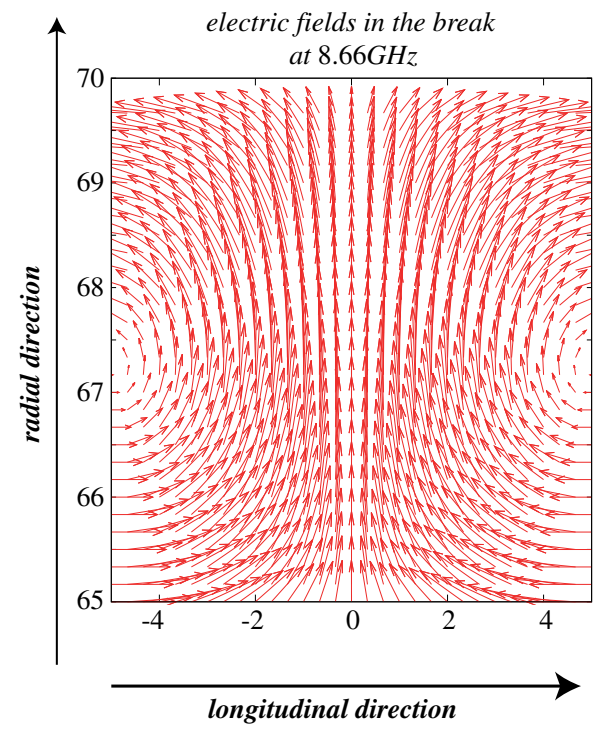

FIG. 7. An example of electric fields in the ceramic break ( $a=65 \mathrm{~mm}, a_{2}=70 \mathrm{~mm}, g=10 \mathrm{~mm}$ and $\epsilon^{\prime}=11$ ) at a cutoff frequency of $8.66 \mathrm{GHz}$. The range of horizontal and that of vertical axes are $-w<z<w$ and $a<\rho<a_{2}$, respectively. Unit is $\mathrm{mm}$.

In Fig. 4, the red, the black and the blue lines show the theoretical, ABCI's and CST's results, respectively. The left and the right parts show the real and the imaginary parts of the impedances, respectively.

In the transverse plane, the real and imaginary parts of impedances are shown in the same figure by the solid and the dot lines, respectively. In Fig. 5, the left part shows only up to $1 \mathrm{GHz}$ frequency, while the right part is up to $12 \mathrm{GHz}$. Note that the scales of the vertical axes are different for the left and right figures. The red, the black and the blue lines show the theoretical, ABCI's and CST's results, respectively.

All the calculation results (the theory, ABCI and CST) reveal the resonance structures and show good agreements between them, in particular, between the theory and $\mathrm{ABCI}$.

The large resonance at low frequency in the transverse impedance (that appears also in the vacuum gap case as shown in the right part of Fig. 2) is modified as

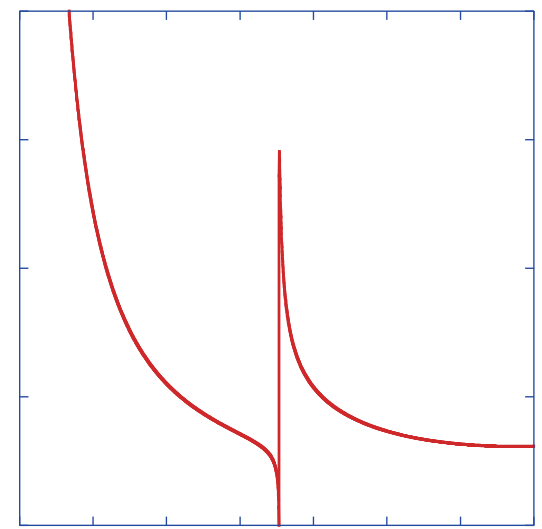

$$
f \sim \frac{c}{2 \pi a} \sqrt{\frac{a^{2}+1.5\left(a_{2}^{2}-a^{2}\right)}{a^{2}+1.5 \epsilon^{\prime}\left(a_{2}^{2}-a^{2}\right)}} .
$$

This frequency is lower than that for the vacuum gap due to the wavelength contraction effects of the ceramic.

In the presence of the ceramic, additional resonances appear at high frequency in the longitudinal as well as in the transverse impedances. Figure 6 presents an example that shows the importance of the correction term [the second terms inside the bracket of Eqs. (7) and (18)]. The red line shows the calculation results when the higher order term up to $m=2$ is included, while the black line shows the results when only the lowest order term is considered. For accurate calculations, the correction term plays an important role. Figure 6 indicates that two types of resonances exist in the impedances.

\section{Cavity-mode resonance}

The first type of resonances is excited around the cutoff frequencies and behaves like cavity-mode resonances. These cutoff frequencies are given by

$$
\begin{aligned}
& f_{c, L}=\frac{j_{0, k} c}{2 \pi a}, \\
& f_{c, T}=\frac{j_{1, k} c}{2 \pi a},
\end{aligned}
$$

for the longitudinal and the transverse impedances, respectively, where $j_{n, k}$ are the $k$ th zeros of $J_{n}(z)$. As shown in Fig. 2, this type of resonances appears also in the vacuum gap case. At the cutoff frequencies, the fields inside the chamber can spread out to infinity and stay there without propagating away.

The electric field pattern in the ceramic break at the frequency of $8.66 \mathrm{GHz}$ (one of the cutoff frequencies) is shown in Fig. 7, where the horizontal and the vertical axes show the longitudinal and the radial directions,

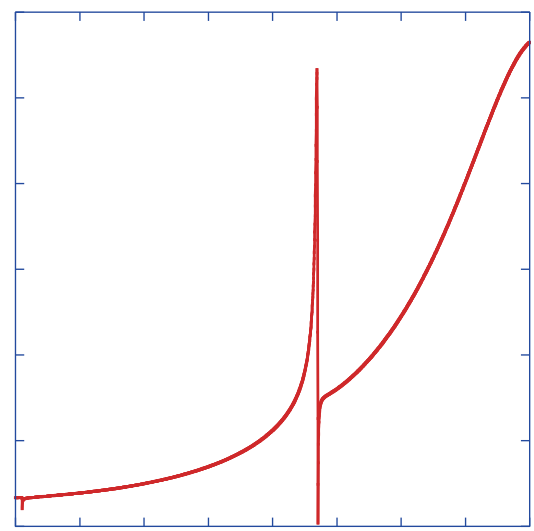

FIG. 8. Two types of resonant structure. 
TABLE I. Resonances and cutoff frequencies for the ceramic break of $\epsilon^{\prime}=11, a=65 \mathrm{~mm}, a_{2}=70 \mathrm{~mm}, g=10 \mathrm{~mm}$.

\begin{tabular}{lcc}
\hline \hline Roots of Eq. (32) & $m=1$ & $9.28, \ldots \mathrm{GHz}$ \\
& $m=2$ & $12.1, \ldots \mathrm{GHz}$ \\
Cutoff frequencies & $1.76,4.05,6.36,8.66,10.96, \ldots \mathrm{GHz}$ \\
$\quad$ for $Z_{L}$ & & \\
Roots of Eq. (33) & $m=1$ & $9.28, \ldots \mathrm{GHz}$ \\
& $m=2$ & $12.1, \ldots \mathrm{GHz}$ \\
Cutoff frequencies & $2.81,5.14,7.47,9.79,12.1, \ldots \mathrm{GHz}$ \\
$\quad$ for $Z_{T}$ & & \\
\hline \hline
\end{tabular}

TABLE II. Resonances and cutoff frequencies for the ceramic break of $\epsilon^{\prime}=11, a=35 \mathrm{~mm}, a_{2}=40 \mathrm{~mm}, g=10 \mathrm{~mm}$.

\begin{tabular}{lcc}
\hline \hline Roots of Eq. (32) & $m=1$ & $9.31, \ldots \mathrm{GHz}$ \\
& $m=2$ & $12.17, \ldots \mathrm{GHz}$ \\
Cutoff frequencies for $Z_{L}$ & $3.27,7.53,11.8, \ldots \mathrm{GHz}$ \\
Roots of Eq. (33) & $m=1$ & $9.33, \ldots \mathrm{GHz}$ \\
& $m=2$ & $12.18, \ldots \mathrm{GHz}$ \\
Cutoff frequencies for $Z_{T}$ & \multicolumn{2}{c}{$5.22,9.55, \ldots \mathrm{GHz}$} \\
\hline \hline
\end{tabular}

respectively. The fields are almost symmetric about the $z=0$ line, and they stay inside the ceramic. Theoretically, the integration of $E_{z}$ along the $z$ direction at $\rho=a$ is almost canceled, and the longitudinal impedance is minimized at this frequency.

In the resonances around the cutoff frequencies, there are two cases. In one case, a resonance appears just above the cutoff frequency (the left part of Fig. 8). In the other case, a resonance appears just below the cutoff frequency (the right part of Fig. 8) [15].

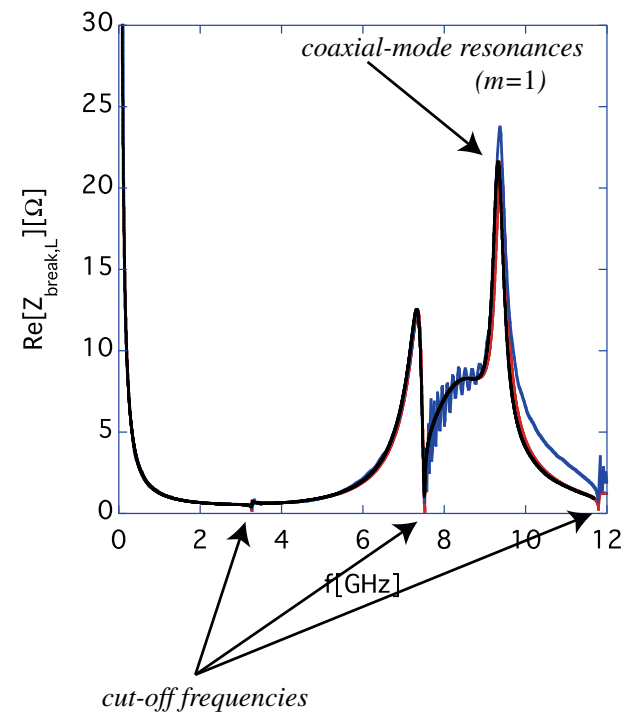

The physical reason for the first case (resonances just above the cutoff frequencies) is as follows. The difference of the dielectric constants between the ceramic and the vacuum introduces the reflection of waves at the boundary. This reflection of electromagnetic fields at the lower and the upper boundaries of the ceramic creates trapped mode inside. These trapped modes can leak out of the ceramic either to the outside or the inside of the chamber. Below the cutoff frequencies, they couple with unpropagating modes inside the chamber, and the interaction between the beam and these unpropagating modes takes place only in a limited space around the ceramic location. Above the cutoff frequencies, however, the trapped modes couple with propagating modes inside the chamber and the interaction between the beam and the propagating modes is extended for much longer distance, and as a result, the impedance is enhanced.

The second case (resonances just below the cutoff frequencies) is excited by another mechanism. Let us simplify the formula of longitudinal impedance for $\left(a_{2}-a\right) / a \ll 1, \quad k w \ll 1$ and $\epsilon^{\prime} \gg 1$. In the case, $A_{0}^{(0)}, C_{0}^{(0)}, I_{0}^{(0)}$ and $K_{0}^{(0)}$ are approximated as

$$
\begin{gathered}
A_{0}^{(0)}=1, \\
C_{0}^{(0)}=j\left(a_{2}-a\right) k \beta Z_{0}, \\
I_{0}^{(0)}=\frac{j\left(a_{2}-a\right) k \beta \epsilon^{\prime}\left(1-\frac{\left(a_{2}-a\right)^{2} k^{2} \beta^{2} \epsilon^{\prime}}{6}\right)}{Z_{0}}, \\
K_{0}^{(0)}=1-\frac{k^{2} \beta^{2} \epsilon^{\prime}\left(a_{2}-a\right)^{2}}{2} .
\end{gathered}
$$

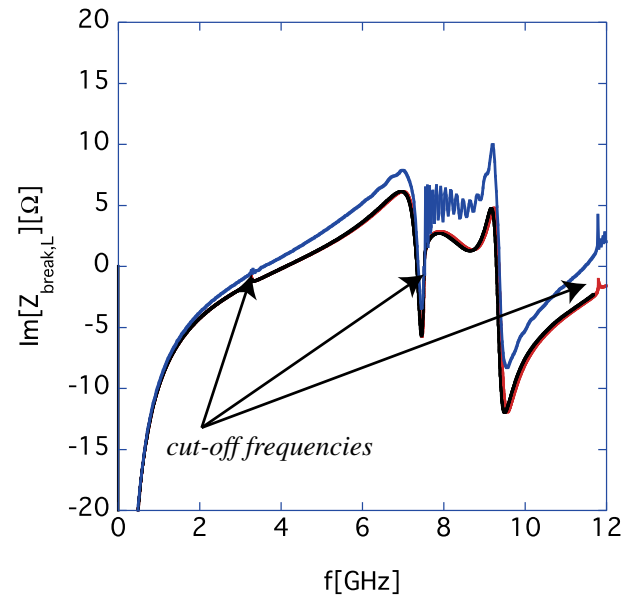

FIG. 9. Longitudinal impedances of the ceramic break $\left(a=35 \mathrm{~mm}, a_{2}=40 \mathrm{~mm}, g=10 \mathrm{~mm}\right.$ and $\left.\epsilon^{\prime}=11\right)$ calculated by the theory (red), the simulation codes ABCI (black) and CST Studio (blue). 

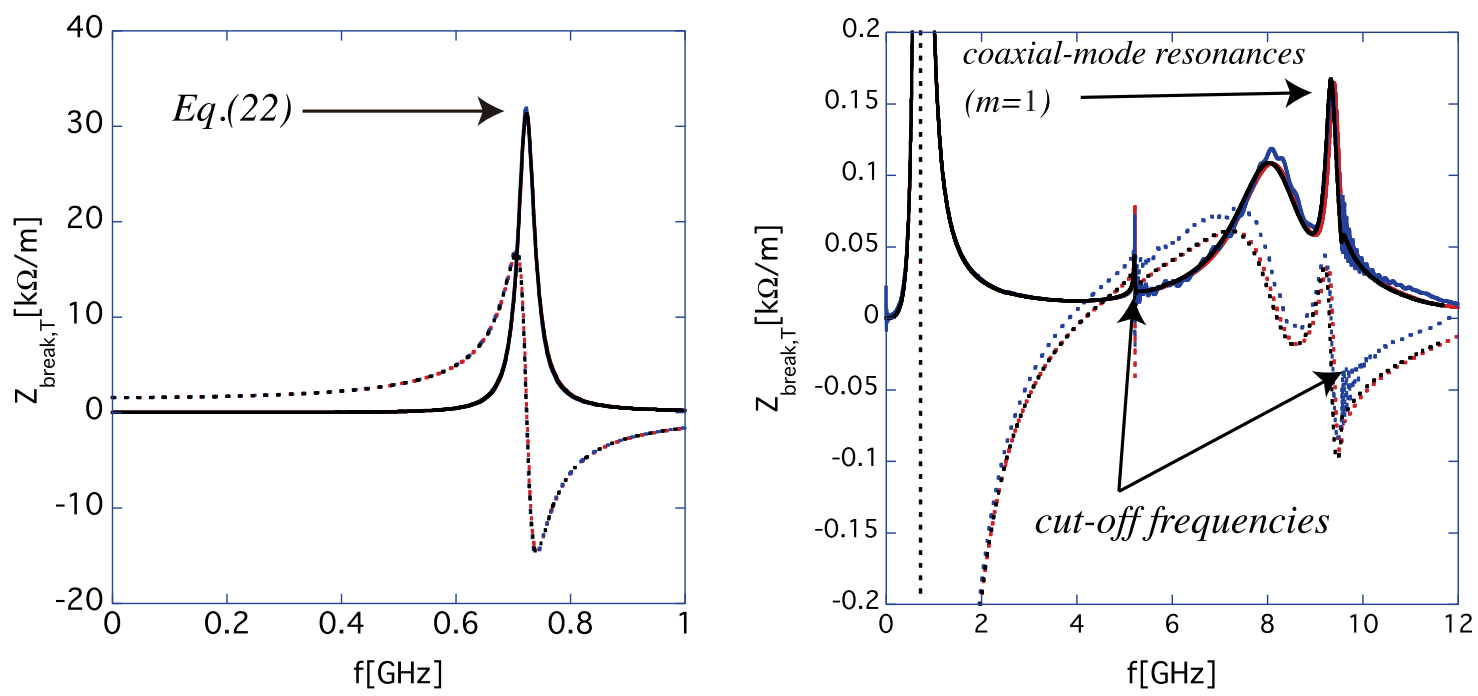

FIG. 10. Transverse impedances of the ceramic break ( $a=35 \mathrm{~mm}, a_{2}=40 \mathrm{~mm}, g=10 \mathrm{~mm}$ and $\left.\epsilon^{\prime}=11\right)$ calculated by the theory (red), the simulation codes ABCI (black) and CST Studio (blue). The solid and the dotted lines show the real and the imaginary parts of the impedance, respectively. The left and the right parts show the results up to $1 \mathrm{GHz}$, and those up to $12 \mathrm{GHz}$, respectively.

Substituting Eqs. (25)-(28) into Eq. (9), the longitudinal impedance of ceramic break is simplified as

$$
Z_{L} \simeq \frac{1}{\frac{j \omega \epsilon^{\prime} \theta_{0} 2 \pi a\left(a_{2}-a\right)}{g} \mathcal{F}+\frac{j k a}{Z_{0}}\left[\langle J\rangle+\frac{\langle Y\rangle}{\left(1-\frac{\left(a_{2}-a\right) g k^{2} \beta^{2}\langle Y\rangle}{2 \pi}-\frac{k^{2} \beta^{2} \epsilon^{\prime}\left(a_{2}-a\right)^{2}}{2}\right)}\right]},
$$

$$
\mathcal{F}=\frac{\left(1-\frac{k^{2} \beta^{2} \epsilon^{\prime}\left(a_{2}-a\right)^{2}}{6}\right)}{\left(1-\frac{\left(a_{2}-a\right) g k^{2} \beta^{2}\langle Y\rangle}{2 \pi}-\frac{k^{2} \beta^{2} \epsilon^{\prime}\left(a_{2}-a\right)^{2}}{2}\right)},
$$

where the mathematical expressions of $\langle J\rangle$ and $\langle Y\rangle$ are given by Eqs. (B5) and (B9), respectively. The first term of the denominator represents the admittance of the ceramic break, because the capacitance $C_{\text {cer }}$ due to the ceramic break is approximately given by

$C_{\mathrm{cer}}=\frac{\epsilon^{\prime} \epsilon_{0} \pi\left(a_{2}+a\right)\left(a_{2}-a\right)}{g} \simeq \frac{\epsilon^{\prime} \epsilon_{0} 2 \pi a\left(a_{2}-a\right)}{g}$,

and the factor $\mathcal{F}$ goes to one at zero frequency. The second term in the denominator of Eq. (29) is the admittance of the gap, or the radiation effects. For a thick gap, the admittance of the ceramic break is capacitive at low and high frequency. However, there is a frequency region between them where the admittance is switched to inductive. On the other hand, the gap itself works as a capacitor below the cutoff frequencies, since the electromagnetic fields can be stored inside without propagating away through the beam chamber. As a result, the coupling between the inductance of the ceramic and the capacitance of the gap creates resonances below the cutoff frequencies in the intermediate frequency region.
One way to check these conjectures is to change the radius of the chamber $a$, and thus the cutoff frequencies. As shown in Tables I and II, the cutoff frequencies of the two chamber cases are quite different. Comparing Figs. 4, 5, and Table I with Figs. 9, 10, and Table II, we notice that the resonance behavior are drastically changed between them.

\section{Coaxial-mode resonance}

As seen in Fig. 6, another type of resonances appears in the ceramic break. This is the coaxial-like mode whose fields are mainly localized inside the ceramic. Their

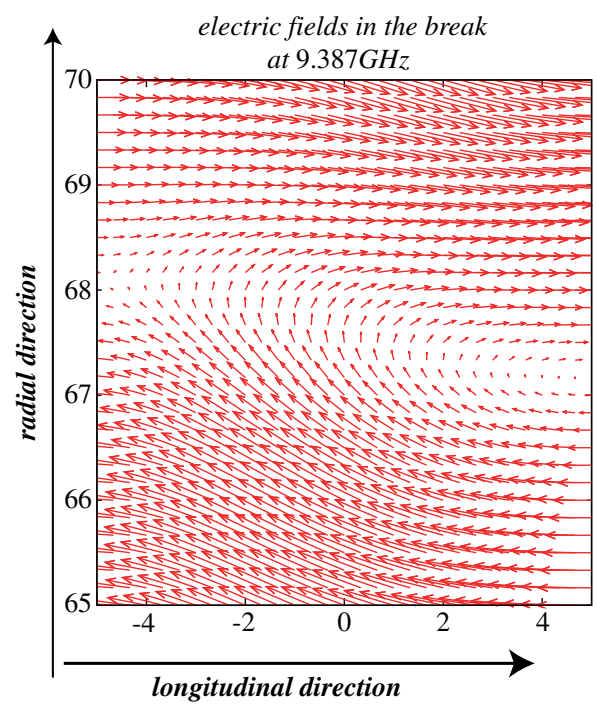

FIG. 11. An example of electric fields in the ceramic break $\left(a=65 \mathrm{~mm}, a_{2}=70 \mathrm{~mm}, g=10 \mathrm{~mm}\right.$ and $\left.\epsilon^{\prime}=11\right)$ at a coaxial-mode resonant frequency of $9.387 \mathrm{GHz}$. The range of horizontal and that of the vertical axes are $-w<z<w$ and $a<\rho<a_{2}$, respectively. Unit is $\mathrm{mm}$. 

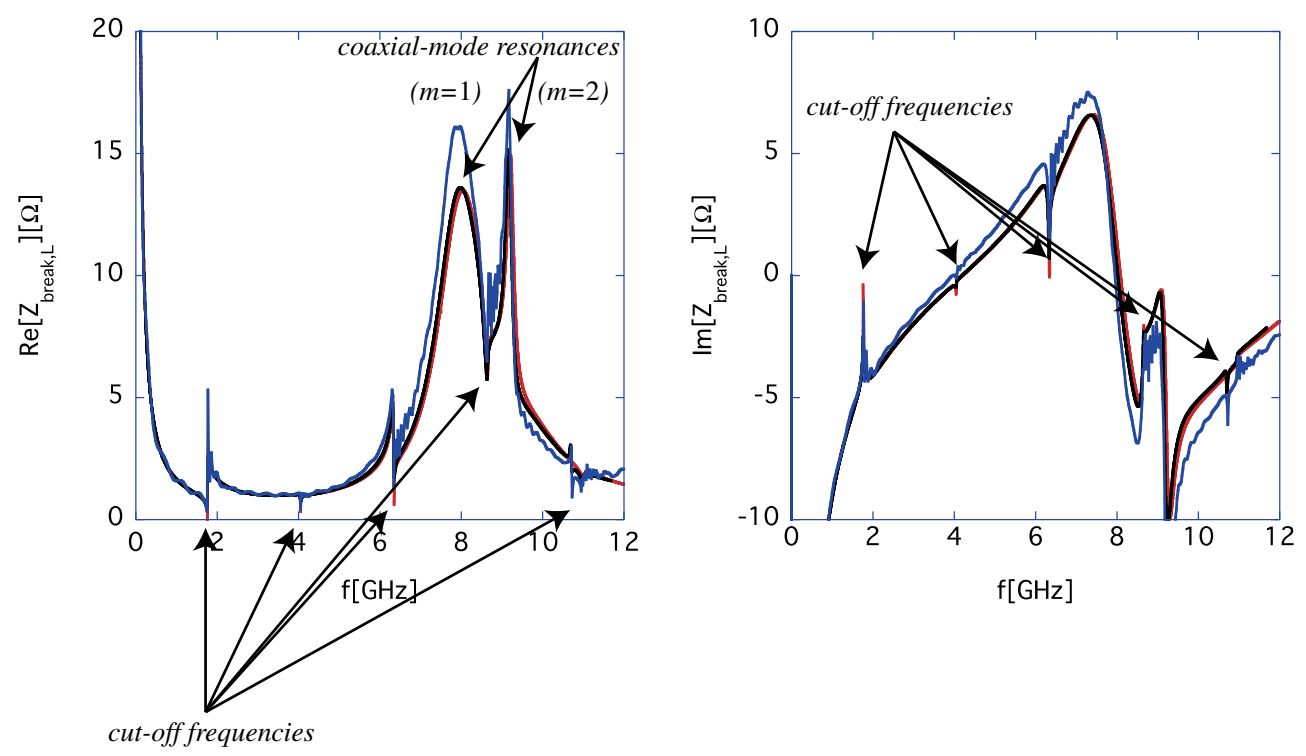

FIG. 12. Longitudinal impedances of the long ceramic break ( $a=65 \mathrm{~mm}, a_{2}=70 \mathrm{~mm}, g=20 \mathrm{~mm}$ and $\left.\epsilon^{\prime}=11\right)$ calculated by the theory (red), the simulation codes ABCI (black) and CST Studio (blue).

resonant frequencies are roughly estimated by the conditions given by

$$
\begin{aligned}
& \frac{\partial\left[Y_{0}^{\prime}\left(\tilde{\mu}_{m} a\right) J_{0}\left(\tilde{\mu}_{m} a_{2}\right)-J_{0}^{\prime}\left(\tilde{\mu}_{m} a\right) Y_{0}\left(\tilde{\mu}_{m} a_{2}\right)\right]}{\partial f}=0, \\
& \frac{\partial\left[Y_{1}^{\prime}\left(\tilde{\mu}_{m} a\right) J_{1}\left(\tilde{\mu}_{m} a_{2}\right)-J_{1}^{\prime}\left(\tilde{\mu}_{m} a\right) Y_{1}\left(\tilde{\mu}_{m} a_{2}\right)\right]}{\partial f}=0,
\end{aligned}
$$

for the longitudinal and the transverse impedances, respectively, where

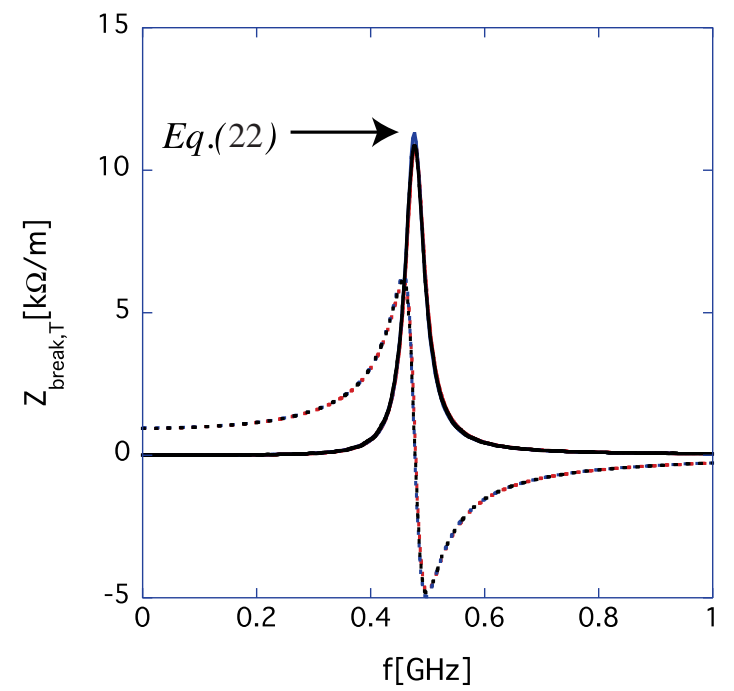

$$
\tilde{\mu}_{m}=\sqrt{\frac{4 \pi^{2} \epsilon^{\prime} f^{2}}{c^{2}}-\frac{m^{2} \pi^{2}}{g^{2}}} .
$$

The positive integer $m$ is a longitudinal (coaxial)-mode number, and denotes the number of half-wavelengths in the ceramic. Figure 11 shows one example of the electric fields in the ceramic at a coaxial-mode resonant frequency of 9.387 GHz. The electric fields are almost parallel to the longitudinal direction at $\rho=a$ and $\rho=a_{2}$. This implies that the fields behave like antenna both on the inner and

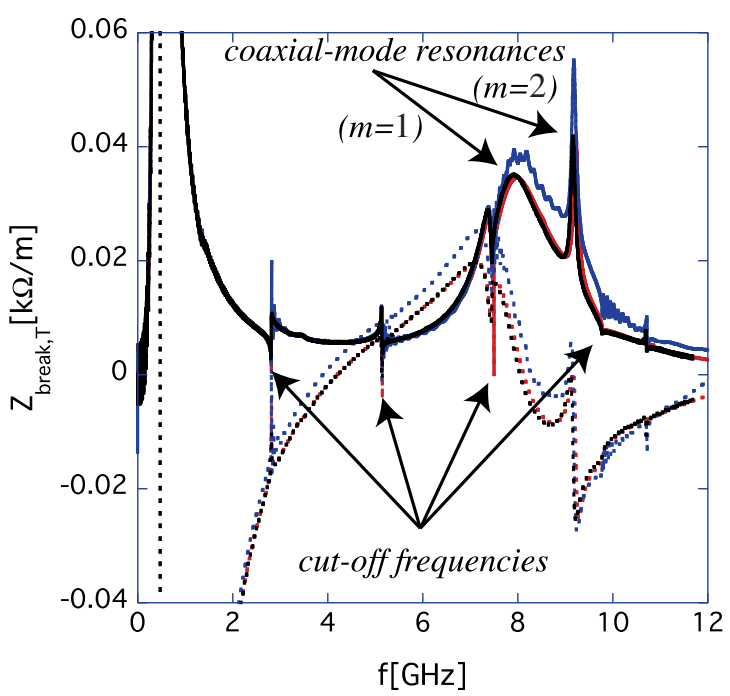

FIG. 13. Transverse impedances for the long ceramic break ( $a=65 \mathrm{~mm}, a_{2}=70 \mathrm{~mm}, g=20 \mathrm{~mm}$ and $\left.\epsilon^{\prime}=11\right)$ calculated by the theory (red), the simulation codes ABCI (black) and CST Studio (blue). The solid and the dotted lines show the real and the imaginary parts of the impedance, respectively. The left and the right parts show the results up to $1 \mathrm{GHz}$, and those up to $12 \mathrm{GHz}$, respectively. 
TABLE III. Resonances and cutoff frequencies for the ceramic break of $\epsilon^{\prime}=11, a=65 \mathrm{~mm}, a_{2}=70 \mathrm{~mm}, g=20 \mathrm{~mm}$.

\begin{tabular}{lcc}
\hline \hline Roots of Eq. (32) & $m=1$ & $8.41, \ldots \mathrm{GHz}$ \\
& $m=2$ & $9.28, \ldots \mathrm{GHz}$ \\
Cutoff frequencies & $1.76,4.05,6.36,8.66,10.96, \ldots \mathrm{GHz}$ \\
$\quad$ for $Z_{L}$ & & \\
Roots of Eq. (33) & $m=1$ & $8.42, \ldots \mathrm{GHz}$ \\
& $m=2$ & $9.28, \ldots \mathrm{GHz}$ \\
Cutoff frequencies & $2.81,5.14,7.47,9.79,12.1, \ldots \mathrm{GHz}$ \\
$\quad$ for $Z_{T}$ & & \\
\hline \hline
\end{tabular}

outer surface of the ceramic, and the fields can propagate away both inside and outside of the chamber. Since the directions of fields are opposite at the inner and outer surfaces, the fields are trapped and resonate in the ceramic. Consequently, the impedance has sharp peaks at their frequencies.

By intentionally changing the maximum order $m$ of correction terms, we can identify which order of coaxial mode is excited in the impedances. In the ceramic break with the values of $a=65 \mathrm{~mm}, a_{2}=70 \mathrm{~mm}$ and $g=10 \mathrm{~mm}$, only the $m=1$ coaxial mode is excited below $12 \mathrm{GHz}$, and the inclusion of up to $m=1$ order in calculations is sufficient for accurate estimates of impedance. On the other hand, the higher order terms (more than $m=2$ ) should be included for accurate estimates of the impedances for long or thick ceramic breaks.

An example of long ceramic break $(g=20 \mathrm{~mm})$ is shown in Figs. 12, 13 and Table III. In these figures, the $m=2$ as well as $m=1$ coaxial modes greatly enhance the impedance, compared with the results of the short ceramic break in Figs. 4 and 5.

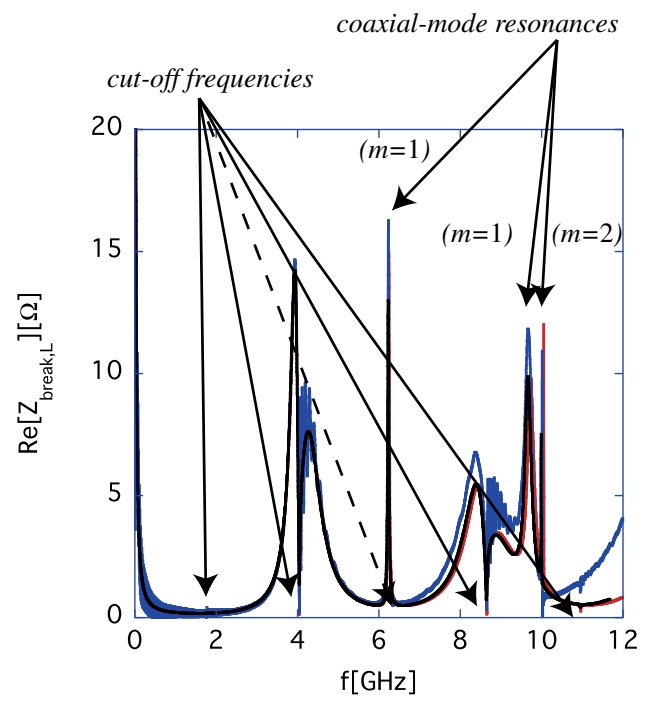

In a thick ceramic with the values of $a=65 \mathrm{~mm}, a_{2}=$ $75 \mathrm{~mm}$ and $g=10 \mathrm{~mm}$ (Fig. 14 and Table IV), we can see three sharp coaxial modes (two $m=1^{\prime}$ s and one $m=2^{\prime}$ s) in the longitudinal impedance. Because of the near-by resonance, the small peak at the cutoff frequency at $6.36 \mathrm{GHz}$ is hidden in Fig. 14. The transverse impedance for this thick ceramic case is not shown here, because ABCI calculation was not possible due to the memory size restriction of the author's computer.

Before finishing this section, let us see how the CST results converge as a function of the total mesh size. Figure 15 shows the CST results of impedance for the ceramic break with $a=65 \mathrm{~mm}, a_{2}=70 \mathrm{~mm}, g=10 \mathrm{~mm}$ and $\epsilon^{\prime}=11$ (the same dimension as in Figs. 4 and 5). The left and the center/right parts correspond to the longitudinal and the transverse impedances, respectively. The black, the blue and the red lines represent the CST results for the total number of mesh sizes to be approximately equal to $2.5 \times 10^{7}, 5 \times 10^{7}$ and $7 \times 10^{7}$, respectively. The solid and the dashed lines are for the real and the imaginary parts of the impedances. As we can see, the blue and the red lines are nearly identical. In all CST calculations, we checked the convergence of the results in this way.

\section{IMPEDANCES OF A CERAMIC BREAK COATED WITH TiN}

In this section, we theoretically analyze the impedances of a ceramic break, where the inner surface of the ceramic is coated with resistive material (TiN) with the conductivity $\left.\sigma_{\mathrm{TiN}}\left(=5.88 \times 10^{6} \mathrm{~S} / \mathrm{m}\right)\right)$ and the thickness $t$. Numerical simulations are not suitable for calculation of this kind of impedance, because extremely small mesh

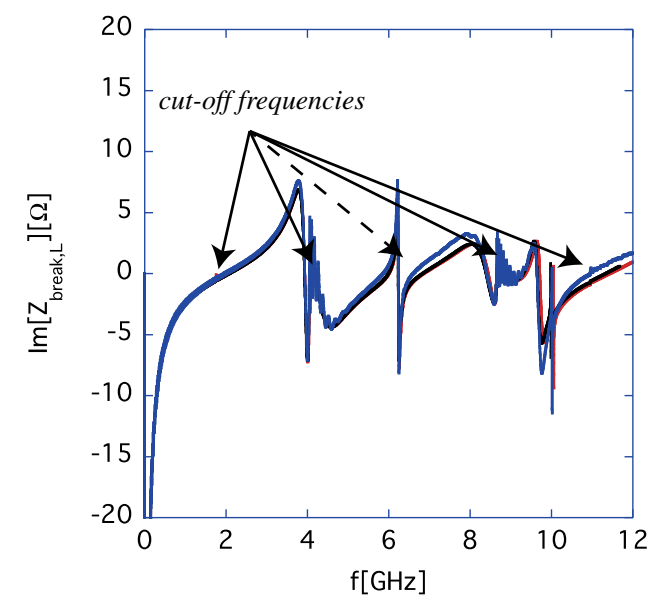

FIG. 14. Longitudinal impedances of the thick ceramic gap ( $a=65 \mathrm{~mm}, a_{2}=75 \mathrm{~mm}, g=10 \mathrm{~mm}$ and $\left.\epsilon^{\prime}=11\right)$ calculated by the theory (red), the simulation codes ABCI (black) and CST Studio (blue). 
TABLE IV. Resonances and cutoff frequencies for the ceramic break of $\epsilon^{\prime}=11, a=65 \mathrm{~mm}, a_{2}=75 \mathrm{~mm}, g=10 \mathrm{~mm}$.

\begin{tabular}{lcc}
\hline \hline Roots of Eq. (32) & $m=1$ & $6.09,9.92, \ldots \mathrm{GHz}$ \\
& $m=2$ & $9.92, \ldots \mathrm{GHz}$ \\
& $1.76,4.05,6.36,8.66,10.96, \ldots \mathrm{GHz}$ \\
Cutoff frequencies & & \\
for $Z_{L}$ & & \\
\hline \hline
\end{tabular}

sizes are necessary (smaller than the thin TiN coating). In reality, typical coating size of $\mathrm{TiN}$ is a few ten $\mathrm{nm}$ in the ceramic break.

In the following subsection, let us investigate the characteristic of the impedance with thin TiN coating.

\section{A. The TiN-thickness dependence of the impedances}

For a thin TiN coating $\left[\kappa_{\mathrm{TiN}} a \gg 1, \kappa_{\mathrm{TiN}} t \ll 1\right.$ and $\left.\left(a_{2}-a\right) / a \ll 1\right]$, the transfer coefficients $A_{0}^{(0)}, C_{0}^{(0)}, I_{0}^{(0)}$ and $K_{0}^{(0)}$ are approximately given by (refer to Appendix C 1)

$$
\begin{gathered}
A_{0}^{(0)}=\cosh \kappa_{\mathrm{TiN}} t+j k \beta a Z_{0} \sigma_{\mathrm{TiN}} t \log \frac{a_{2}}{a}, \\
C_{0}^{(0)}=j k \beta a Z_{0} \log \frac{a_{2}}{a}, \\
I_{0}^{(0)}=\frac{j \beta \epsilon^{\prime} k\left(a_{2}^{2}-a^{2}\right) \cosh \kappa_{\mathrm{TiN}} t}{2 a_{2} Z_{0}}+\frac{a \kappa_{\mathrm{TiN}} \sinh \kappa_{\mathrm{TiN}} t}{a_{2} j k \beta Z_{0}}, \\
K_{0}^{(0)}=-\frac{\epsilon^{\prime} k^{2} \beta^{2}\left(a_{2}^{2}-a^{2}\right) t}{2 a_{2}}+\frac{a}{a_{2}} \cosh \kappa_{\mathrm{TiN}} t,
\end{gathered}
$$

$$
\kappa_{\mathrm{TiN}}=\sqrt{j k \beta Z_{0} \sigma_{\mathrm{TiN}}} .
$$

Substituting Eqs. (35)-(38) into Eq. (9), the longitudinal impedance $Z_{\text {cerTiN, } L}$ of the thin ceramic break (compared with the radius of the chamber) coated with the thin TiN is approximated as

$$
Z_{\mathrm{cerTiN}, L} \simeq \frac{\frac{4 a_{2} I_{1}^{2}(\bar{k} \sigma)}{\sigma^{2} \bar{k}^{2} a I_{0}^{2}(\bar{k} a)}\left(\frac{\sin k w}{k w}\right)^{2}}{\frac{j k \beta a_{2}[\langle J(z)\rangle+\langle Y(z)\rangle]}{Z_{0}}-\frac{j \pi a_{2} \kappa_{\mathrm{TiN}} \tanh \kappa_{\mathrm{TiN}} t}{w k \beta Z_{0}}+\frac{j \omega \epsilon^{\prime} \pi a_{2}\left(a_{2}-a\right)}{c Z_{0} w}} .
$$

The first term in the denominator is the admittance of the gap, or radiation effects. The second term represents the resistive wall admittance due to the TiN coating. The third term in the denominator is the admittance of the ceramic break. The longitudinal impedance of the ceramic break is approximately obtained by adding the impedances in parallel (the impedance of resistive wall due to the TiN coating, that of the radiation and that of the capacitor made by the ceramic).

More rigorous calculations can be numerically done by using Eqs. (C21)-(C24) in Appendix C 1 as transfer coefficients. The longitudinal impedance is obtained by solving Eqs. (A20)-(A23) in Appendix A 1 with the transfer functions and by substituting the solutions $\left(V_{1}\right.$ and $\left.V_{1}^{(m)}\right)$ into Eq. (7).

With the help of the discussion of the previous study [11], let us categorize the impedances of the ceramic break with the TiN coating for various TiN thickness. The frequency parameter $f_{\delta}$ is introduced as

$$
f_{\delta} \equiv \frac{c}{\pi Z_{0} \sigma_{c} t^{2}}, \quad \text { (the frequency at which the skin depth is equal to the TiN thickness), }
$$
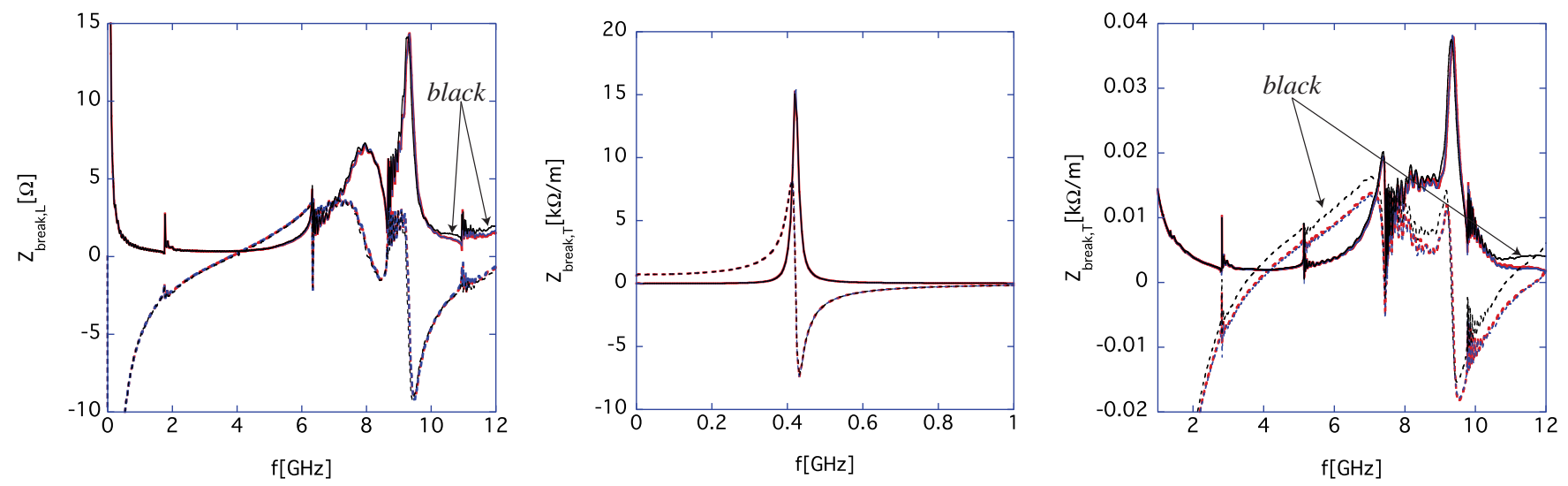

FIG. 15. The results of CST Studio for different total number of meshes are shown by the black $\left(\sim 2.5 \times 10^{7}\right)$, the blue $\left(\sim 5 \times 10^{7}\right)$ and the red $\left(\sim 7 \times 10^{7}\right)$ lines. The left part shows the longitudinal impedance. The center $(0-1 \mathrm{GHz})$ and right $(1-12 \mathrm{GHz})$ parts show the transverse impedances. The solid and the dashed lines show the real and the imaginary parts of the impedances. 
and the thickness parameter $t_{\min }$ is as

$$
t_{\min } \equiv\left(\frac{4 g}{\pi^{2} Z_{0}^{3} \sigma_{c}^{3}}\right)^{\frac{1}{4}}(\sim \text { typically of the order of a few ten } \mathrm{nm} \text { in the short ceramic break). }
$$

The left part of Fig. 16 shows the TiN thickness dependence of the longitudinal impedance. In all the cases, the TiN coating is sufficiently thin so that its thickness is less than the skin depth within the frequency frame of the figures $(f<10 \mathrm{GHz})$ except the $t=10 \mu \mathrm{m}$ case where the skin depth starts to fall short of the TiN thickness above $f_{\delta}(\sim 1 \mathrm{GHz})$. When the TiN coating is thick enough $\left(t>t_{\min }\right)$, but less than the skin depth, the entire image current runs on the TiN coating, and the impedance becomes proportional to $1 / t$. Since the TiN coating almost perfectly shields the electromagnetic fields inside the beam chamber from leaking out to the ceramic, the appearance of resonances inside the ceramic is greatly suppressed. When the TiN coating is much thinner than $t_{\min }$ (e.g., $t<1 \mathrm{~nm}$ ), the electromagnetic fields starts to leak out through the TiN coating, and the existence of the ceramic and their resonance structures starts to form the impedance.

Similarly to the longitudinal case, the transverse impedance is proportional to $1 / t$ for sufficiently thick TiN coating $\left(t>t_{\min }\right)$, but thinner than the skin depth below $f_{\delta}$. When the TiN coating is extremely thin $\left(t<t_{\min }\right)$, the shielding effect of the TiN coating starts to diminish, and resonance structures start to appear.

Similarly to the longitudinal impedance, let us try to obtain a simple expression of the transverse impedance of the ceramic break with the thin TiN coating. When $\kappa_{\mathrm{TiN}} a \gg 1$, $\kappa_{\mathrm{TiN}} t \ll 1$ and $\left(a_{2}-a\right) / a \ll 1$, the coefficients $A_{1}^{(0)}, C_{1}^{(0)}, I_{1}^{(0)}$ and $K_{1}^{(0)}$ are approximated as (refer to Appendix C 2)

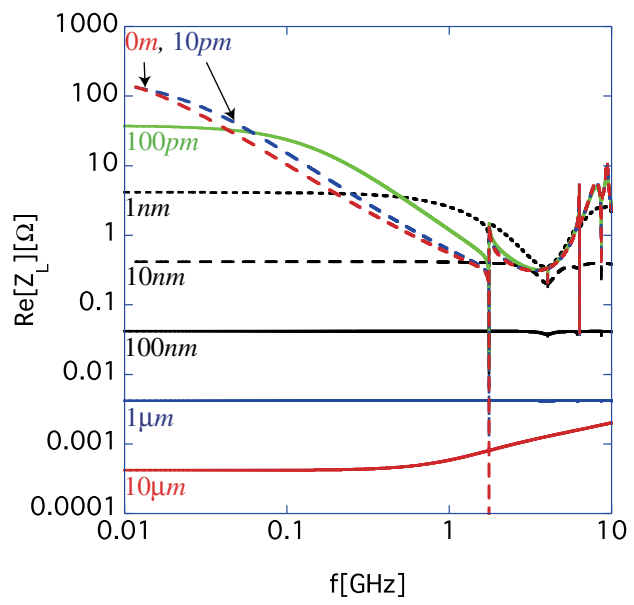

$$
\begin{gathered}
A_{1}^{(0)} \simeq \cosh \kappa_{\mathrm{TiN}} t, \\
C_{1}^{(0)} \simeq 0, \\
I_{1}^{(0)} \simeq \frac{\kappa_{\mathrm{TiN}}}{j k \beta Z_{0}} \sinh \kappa_{\mathrm{TiN}} t \\
+\frac{j k \beta \epsilon^{\prime}\left(a_{2}-a\right)}{Z_{0}}\left(1-\frac{1}{a^{2} k^{2} \beta^{2} \epsilon^{\prime}}\right) \cosh \kappa_{\mathrm{TiN}} t, \\
K_{1}^{(0)} \simeq \cosh \kappa_{\mathrm{TiN}} t .
\end{gathered}
$$

In the lowest order approximation, the transverse impedance of the ceramic break $Z_{\text {certain, } T}$ coated with TiN, is expressed as

$$
Z_{\text {certain }, T}=\frac{a_{2} I_{1}\left(\bar{k} r_{b}\right)}{r_{b} a \gamma I_{1}^{2}(\bar{k} a) \tilde{\mathcal{V}}_{3, b}}\left(\frac{\sin k w}{k w}\right)^{2},
$$

where

$$
\begin{aligned}
\tilde{\mathcal{V}}_{3, b}= & -\frac{j \pi a_{2} \kappa_{\mathrm{TiN}} \tanh \kappa_{\mathrm{TiN}} t}{w k \beta Z_{0}} \\
& -\frac{j k \beta a_{2}\left[\left\langle Y_{2}(z)-\frac{Y_{3}(z)}{k^{2} \beta^{2} a^{2}}\right\rangle-\left\langle Y_{5}(z)-\frac{Y_{6}(z)}{k^{2} \beta^{2} a_{2}^{2}}\right\rangle\right]}{Z_{0}} \\
& +\frac{\pi j \beta \epsilon^{\prime} k\left(a_{2}-a\right) a_{2}}{w Z_{0}}\left(1-\frac{1}{a^{2} k^{2} \beta^{2} \epsilon^{\prime}}\right) .
\end{aligned}
$$

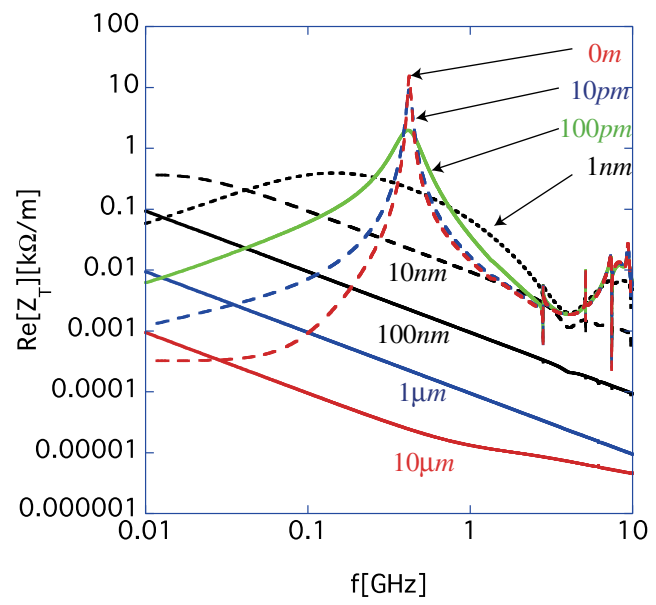

FIG. 16. The thickness dependence on the impedances of the ceramic break $\left(\epsilon^{\prime}=11, a=65 \mathrm{~mm}, a_{2}=70 \mathrm{~mm}\right.$ and $\left.g=10 \mathrm{~mm}\right)$ with the TiN coating. The impedances for the different TiN thicknesses are shown by the red solid $(t=10 \mu \mathrm{m})$, the blue solid $(t=1 \mu \mathrm{m})$, the black solid $(t=100 \mathrm{~nm})$, the black dashed $(t=10 \mathrm{~nm})$, the black dot $(t=1 \mathrm{~nm})$, the green solid $(t=100 \mathrm{pm})$, the blue dashed $(t=10 \mathrm{pm})$ and the red dashed $(t=0 \mathrm{~m})$ lines. 
The first, the second and the third terms represent the admittances of the resistive wall due to the TiN coating, the radiation effect and the ceramic break, respectively. The admittance made by the ceramic break (the third term) is modulated at low frequency. Roughly, the transverse impedance can be interpreted as the summation of three components in parallel (the resistive wall due to the TiN coating, the radiation and the capacitor made by ceramic), again.

Specifically for a vacuum gap made of two infinitesimal thickness chambers, Eq. (47) reproduces the gap impedance $Z_{\mathrm{gap}, \perp}$,

$$
Z_{\text {gap }, \perp} \simeq-\frac{j Z_{0} I_{1}\left(\bar{k} r_{b}\right)}{\gamma k \beta r_{b} a I_{1}^{2}(\bar{k} a)\left[Y_{5}\left(z_{0}\right)-Y_{2}\left(z_{0}\right)-\frac{Y_{6}\left(z_{0}\right)-Y_{3}\left(z_{0}\right)}{k^{2} \beta^{2} a^{2}}\right]},
$$

in Ref. [10], where $z_{0}$ is a matching point $\left(-w<z_{0}<w\right)$.

\section{B. Ceramic length dependence of the impedance}

Let us study how the impedance of the ceramic break with the TiN coating will be changed when the length of the ceramic is changed. Figure 17 shows such an example, where the inner radius of the ceramic $a=65 \mathrm{~mm}$, the outer radius $a_{2}=70 \mathrm{~mm}$ and the TiN thickness $t=15 \mathrm{~nm}$. The red, the blue and the black lines show the longitudinal impedance per unit length for $g=10 \mathrm{~mm}, 100 \mathrm{~mm}$ and $1 \mathrm{~m}$, respectively. The solid and the dashed lines show the real and the imaginary parts of the impedances, respectively. One can find that the longitudinal impedance per

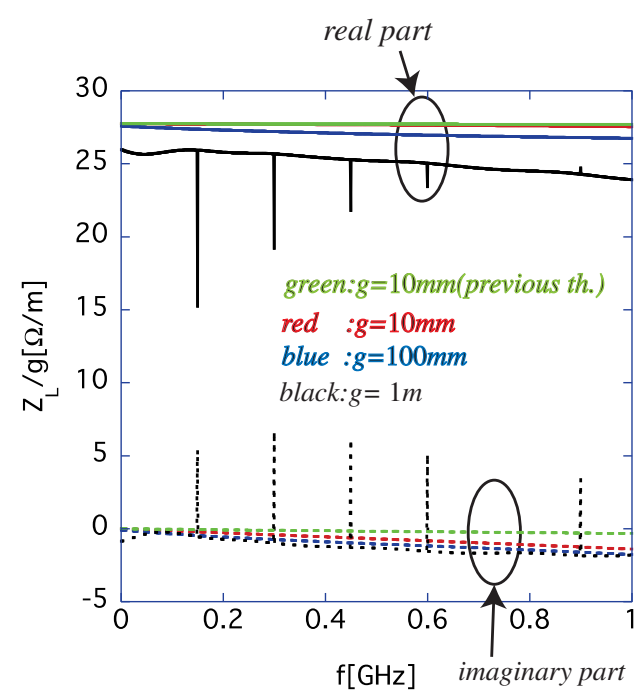

FIG. 17. The longitudinal impedances per unit length of the ceramic break ( $a=65 \mathrm{~mm}, a_{2}=70 \mathrm{~mm}$ and $\left.\epsilon^{\prime}=11\right)$ with the TiN coating $(15 \mathrm{~nm})$ are shown by the red $(g=10 \mathrm{~mm})$, the blue $(g=100 \mathrm{~mm})$ and the black $(g=1 \mathrm{~m})$ lines. The green line shows the results $(g=10 \mathrm{~mm})$ given by the previous short insert theory [11]. The solid and the dashed lines show the real and the imaginary parts of the impedances. unit length hardly varies as long as the ceramic length is less than or comparable to the beam pipe radius (compare the nearly identical results for the $g=10 \mathrm{~mm}$ and the $g=100 \mathrm{~mm}$ cases). No resonance structure is visible. This is because the entire image current still runs on the TiN coating, making it as a perfect rf shield to block the wakefield inside the beam chamber from reaching the ceramic. However, once the ceramic becomes much longer than the chamber dimension (for example, the $g=1 \mathrm{~m}$ case), only a partial image current runs on the TiN coating, and the shielding effect is weakened. As a result, the resonance structure starts to appear.

The green lines show the result for the $g=10 \mathrm{~mm}$ case calculated using the previous short insert theory [11], where only a short thin resistive insert is sandwiched by two thin metals. While the agreement between the present and previous theories for the real parts of the impedance is good, the imaginary parts of the impedance reveal discrepancy at high frequency. This is because the previous theory assumes a thin short insert to make an approximation of the parallel (to the longitudinal direction) electric fields inside the gap. This approximation is no longer too good for the present $5 \mathrm{~mm}$ thick ceramic. One can get a good agreement between the present and the previous theories when the ceramic thickness is reduced to $1 \mathrm{~mm}$ (see Fig. 18). On the other hand, the present theory makes no such assumption or approximation, and is applicable to any size of the ceramic break.

\section{The dependence of the longitudinal impedance on the boundary conditions}

It seems that some of the present results look contradictory to some previous studies related to the ceramic

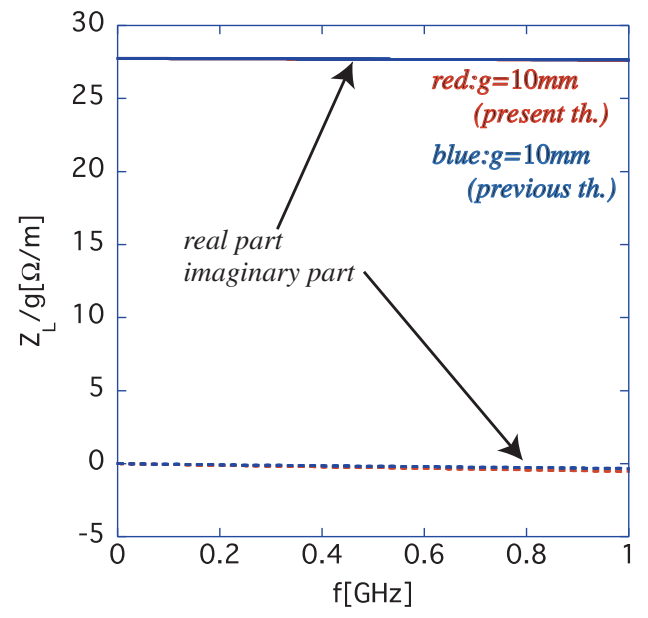

FIG. 18. The longitudinal impedances per unit length of the ceramic break ( $a=65 \mathrm{~mm}, a_{2}=66 \mathrm{~mm}, g=10 \mathrm{~mm}$ and $\left.\epsilon^{\prime}=11\right)$ with the TiN coating $(15 \mathrm{~nm})$. The red and the blue lines show the results given by the present theory and the previous short insert theory [11]. The solid and the dashed lines show the real and the imaginary parts of the impedances. 
chambers $[4,15-19]$ where the real part of the longitudinal impedance goes to zero at zero frequency [refer to Eq. (56)]. However, in their studies, the (finite) element (or ceramic chamber) is surrounded by perfectly conductive walls, which is equivalent to that $V_{2}$ and $V_{2}^{(m)}$ are identical to zero in this report. To simulate this case, the boundary conditions given by Eqs. (A20)-(A23) in Appendix A 1 are modified as

$$
\begin{gathered}
0=A_{0}^{(0)} \frac{V_{1}}{2 w}+C_{0}^{(0)}\left(\langle\alpha(z)\rangle+\frac{V_{1}}{2 \pi} \frac{j k \beta}{Z_{0}}\langle J(z)\rangle+\frac{k \beta}{2 \pi w Z_{0}} \sum_{m=1}^{\infty} V_{1}^{(m)}\left\langle J^{(m)}(z)\right\rangle\right), \\
0=A_{0}^{(m)} V_{1}^{(m)}+C_{0}^{(m)}(\langle\alpha(z)\rangle\rangle_{m}+\frac{j k \beta}{2 \pi Z_{0}} V_{1}\left\langle\langle J(z)\rangle_{m}+\frac{k \beta}{2 \pi w Z_{0}} \sum_{n=1}^{\infty} V_{1}^{(n)}\left\langle\left\langle J^{(n)}(z)\right\rangle\right\rangle_{m}\right) .
\end{gathered}
$$

For infinitely long chambers, $V_{1}$ and $V_{1}^{(m)}$ are solved as

$$
\begin{gathered}
V_{1}=-\frac{2 C_{0}^{(0)} \beta c I_{1}(\bar{k} \sigma)}{\left(A_{0}^{(0)}+\frac{j C_{0}^{(0)} J_{1}(k \beta a)}{Z_{0} J_{0}(k \beta a)}\right) \pi \sigma \bar{k} a I_{0}(\bar{k} a)} \frac{\sin k w}{k}, \\
V_{1}^{(m)}=\frac{k \beta c I_{1}(\bar{k} \sigma) C_{0}^{(m)}}{j \pi \sigma \bar{k} a I_{0}(\bar{k} a)\left(A_{0}^{(m)}+\frac{C_{0}^{(m)} j \beta \gamma I_{1}(\bar{k} a)}{Z_{0} I_{0}(\bar{k} a)}\right)} \frac{\left[(-1)^{m} e^{-j k w}-e^{j k w}\right]}{\left(k^{2}-\frac{m^{2} \pi^{2}}{4 w^{2}}\right)},
\end{gathered}
$$

using Eq. (B1) in Appendix B.

Substituting Eq. (53) into Eq. (7), we obtain the expression of longitudinal impedance for an infinitely long ceramic break as

$$
Z_{\text {break }, L} \simeq \frac{4 w I_{1}^{2}(\bar{k} \sigma) C_{0}\left(a_{2}, a\right)}{\pi \sigma^{2} \bar{k}^{2} a I_{0}^{2}(\bar{k} a)\left[A_{0}\left(a_{2}, a\right)+\frac{C_{0}\left(a_{2}, a\right) j \beta \gamma I_{1}(\bar{k} a)}{Z_{0} I_{0}(\bar{k} a)}\right]},
$$

where

$$
\sum_{m=1}^{\infty} \frac{2-(-1)^{m} e^{j 2 k w}-(-1)^{m} e^{-j 2 k w}}{\left(k^{2}-\frac{m^{2} \pi^{2}}{4 w^{2}}\right)^{2}}=\frac{\cos 2 k w-1}{k^{4}}+\frac{2 w^{2}}{k^{2}}
$$

is used and the coefficients $A_{0}\left(a_{2}, a\right)$ and $C_{0}\left(a_{2}, a\right)$ are given by Eqs. (E12) and (E13), respectively. Equation (54) is identical to Eq. (E19) of the Appendix E, where the infinitely long chamber with the TiN coating is assumed from the beginning. Specifically, at low frequency for a thin TiN coating, we can approximately reproduce the formula [17] from Eq. (54) as

$$
Z_{L} \simeq \frac{w Z_{0}}{\pi \beta a\left(Z_{0} t \sigma_{\mathrm{TiN}}-\frac{j \epsilon^{\prime}}{\left(\epsilon^{\prime} \beta^{2}-1\right) k a \log \frac{a_{2}}{a}}\right)} .
$$

This result indicates that it is the perfectly conductive boundary condition that makes the impedance zero at zero frequency.
Another recent study [20] shows that the impedances of an infinitely long, axisymmetrical beam pipe (made by multilayered materials) diminishes at zero frequency. In this axisymmetrical beam pipe, it is assumed implicitly that the entire system is uniformly surrounded by a perfectly conductive wall in the far distance. Therefore, for the same reason as in above, the longitudinal impedance diminishes at zero frequency.

From these considerations, we can conclude that the different behaviors of the longitudinal impedances near the zero frequency come from the different assumptions of the system configuration under consideration.

\section{The dependence on the TiN thickness when the ceramic break is covered by a perfectly conductive wall}

In this section, let us consider how the impedance depends on the TiN-thickness $t$, when the ceramic break is covered by the perfectly conductive wall on its outer surface. Figure 19 shows the real parts of the impedances at $f=1.2 \mathrm{MHz}$, normalized by the gap length $g$. The red solid and the blue dashed lines in the left part correspond to the $g=10 \mathrm{~mm}$ and $g=1 \mathrm{~m}$, respectively. Their agreement shows that the impedance is simply proportional to the gap length.

All results in the right part correspond to those for $g=$ $10 \mathrm{~mm}$ case. The red solid, the blue solid and the black dashed lines in the right part show the results obtained by solving Eqs. (50) and (51), by Eq. (E19) of the Appendix E, and by the approximate result Eq. (56), respectively. For reference, the green $(g=10 \mathrm{~mm})$ and the brown dashed $(g=1 \mathrm{~m})$ lines show the cases where the ceramic is not surrounded by the walls. When the TiN thickness is sufficiently larger than the skin depth, the impedance is determined by the skin depth, and has no TiN thickness dependence (shown by the flat red and blue lines at large $t$ region). In the thin TiN coating region, the rigorous solution for the infinitely long ceramic (the blue line) agrees well with its approximate solution (the black dashed line). However, they start to deviate at the thick TiN region (because the approximation is only valid when the skin 

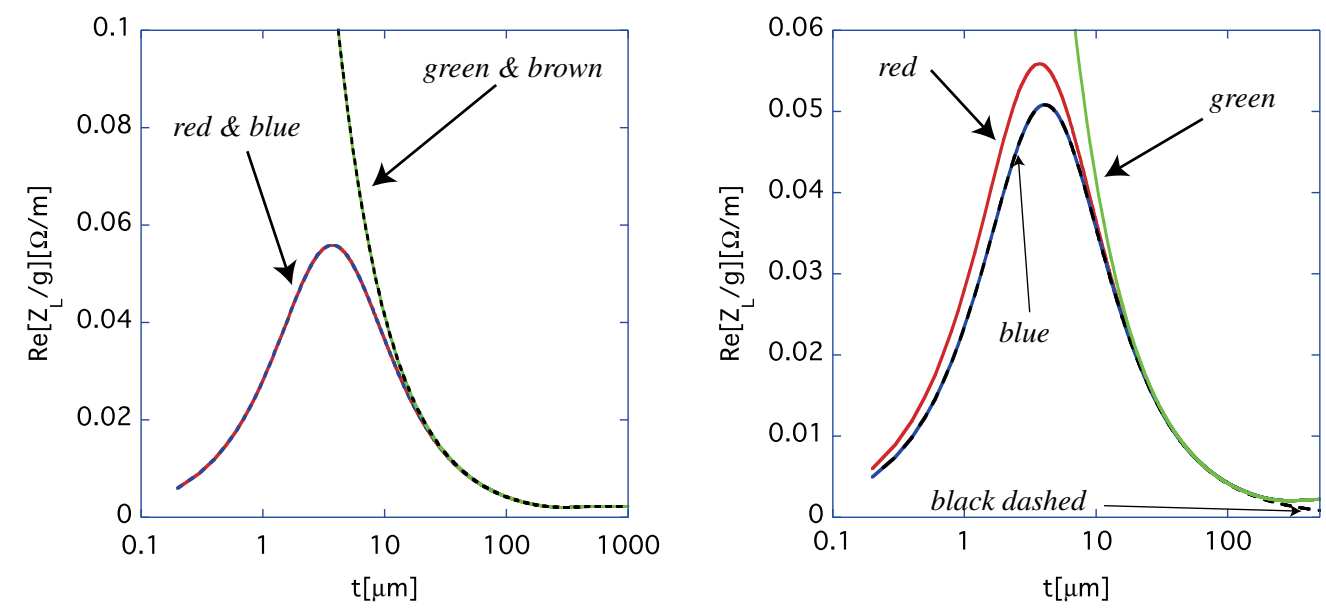

FIG. 19. The TiN thickness $t$ dependence of impedances per unit length at $1.2 \mathrm{MHz}$, where the ceramic chamber $(a=65 \mathrm{~mm}$, $a_{2}=70 \mathrm{~mm}$ and $\epsilon^{\prime}=11$ ) is coated with the TiN. The left part shows the impedances for $g=10 \mathrm{~mm}$ (red solid) and for $g=1 \mathrm{~m}$ (blue dashed). The right part shows the impedances $(g=10 \mathrm{~mm}$ ) obtained by solving Eqs. (50) and (51) (red solid), by Eq. (E19) (blue solid) and by Eq. (56) (black dashed). The red, blue and black lines correspond to the case when the ceramic is surrounded by perfectly conductive walls. The green $(g=10 \mathrm{~mm})$ and the brown dashed $(g=1 \mathrm{~m})$ lines that correspond to the case where the ceramic is not surrounded by the walls are also shown as references.

depth is larger than the thickness $t$ ). On the other hand, the complete solution (the red line) shows larger impedance values in the range where the TiN coating is smaller than $10 \mu \mathrm{m}$, since it includes the contributions of the fields trapped inside the ceramic sandwiched by the perfectly conductive chambers on both sides. In this case, it is observed that the longitudinal impedance is maximum at a given TiN thickness (several $\mu \mathrm{m}$ in the present example). The theory predicts a thinner TiN coating is preferable from the impedance point of view, as long as it is below that several $\mu \mathrm{m}$.

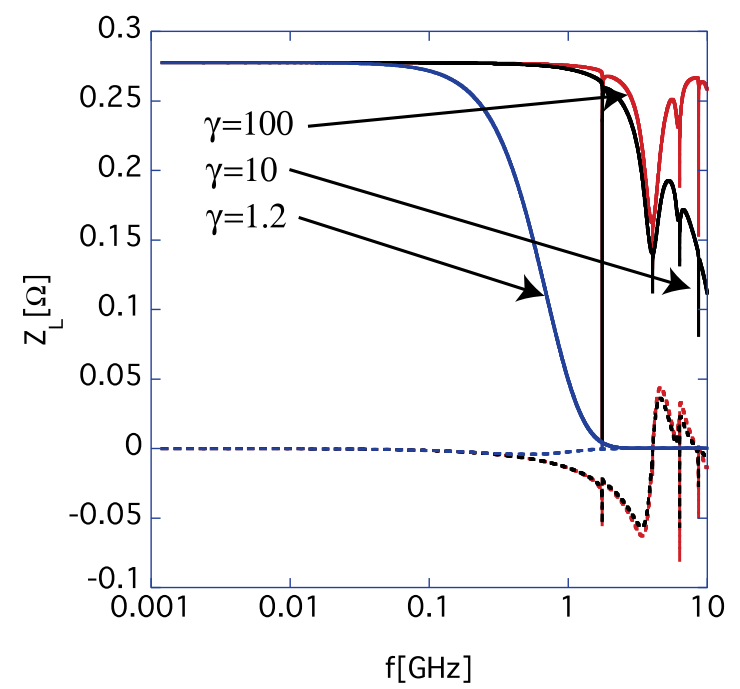

\section{THE LORENTZ- $\gamma$ FACTOR DEPENDENCE OF IMPEDANCES}

Finally, let us investigate the Lorentz- $\gamma$ factor dependence of the impedances of the ceramic break with and without TiN coating. The present theory is applicable to nonrelativistic beams, because it makes no approximation on the energy of the beam. Figures 21 and 20 demonstrate the Lorentz- $\gamma$ factor dependence with and without TiN coating, respectively. The red, the black and the blue lines show the result for $\gamma=100, \gamma=10$ and $\gamma=1.2$, respectively. The left and the right parts show the longitudinal and

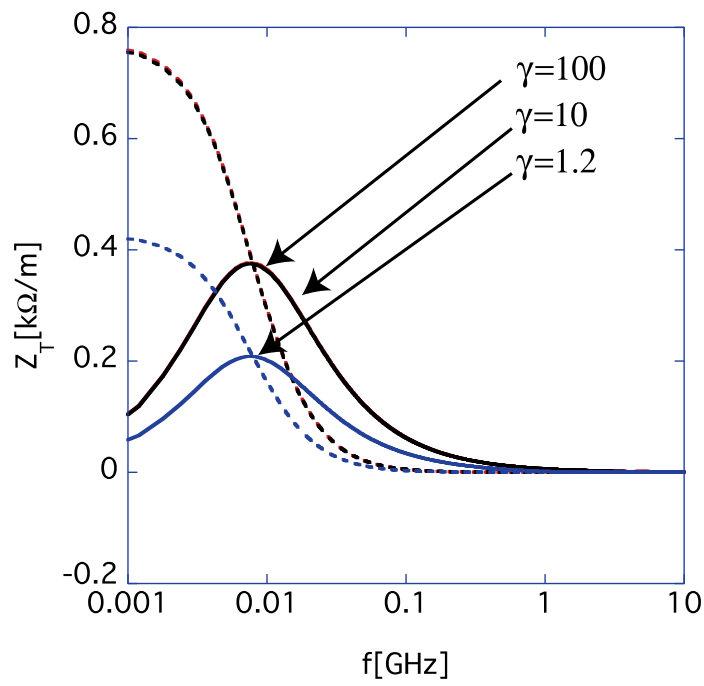

FIG. 20. The impedances of the ceramic break ( $a=65 \mathrm{~mm}, a_{2}=70 \mathrm{~mm}$ and $\left.\epsilon^{\prime}=11\right)$ with the TiN coating (15 nm) for different Lorentz- $\gamma$ are shown by the red $(\gamma=100)$, the black $(\gamma=10)$ and the blue $(\gamma=1.2)$ lines. The solid and the dashed lines show the real and the imaginary parts of the impedances, respectively. 

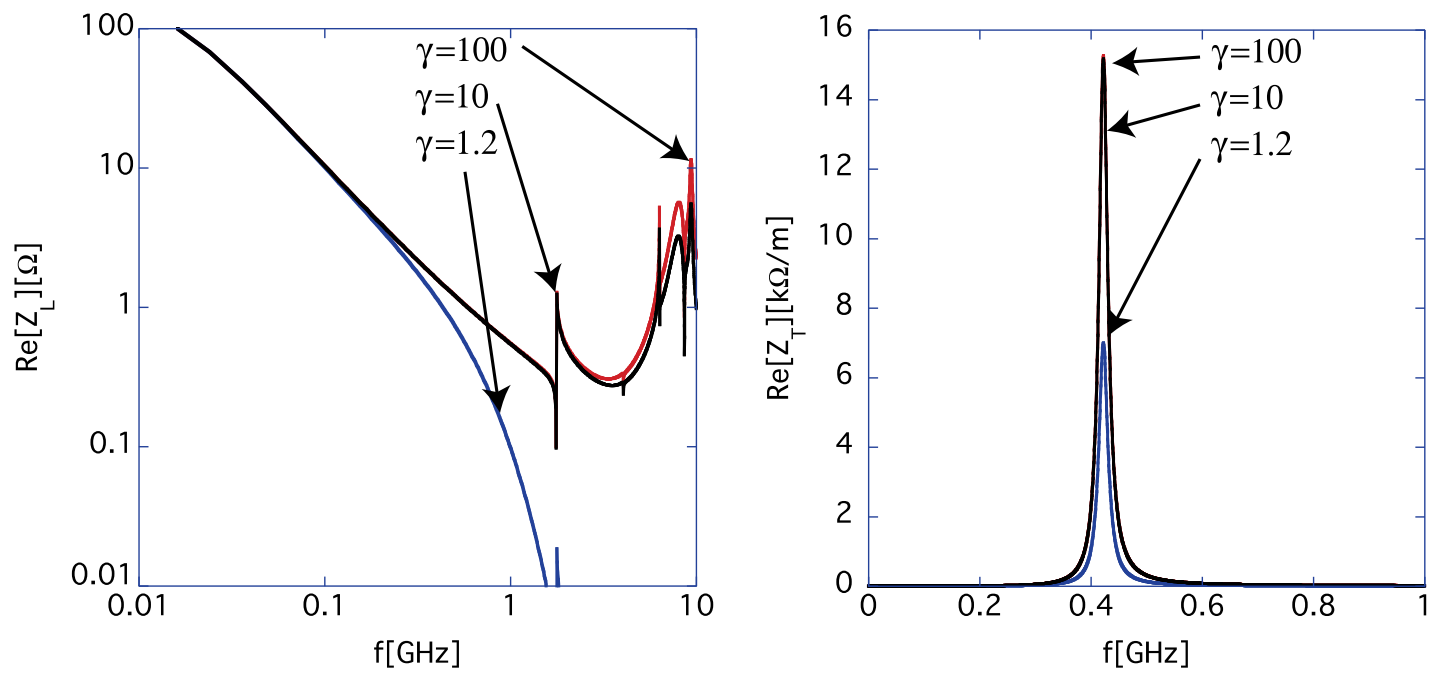

FIG. 21. The impedances of the ceramic break ( $a=65 \mathrm{~mm}, a_{2}=70 \mathrm{~mm}$ and $\left.\epsilon^{\prime}=11\right)$ without the TiN coating for different Lorentz- $\gamma$ factors are shown by the red $(\gamma=100)$, the black $(\gamma=10)$ and the blue $(\gamma=1.2)$ lines.

the transverse impedances, respectively. The solid and the dashed lines show the real and the imaginary parts of the impedances, respectively.

Generally speaking, the impedance for a nonrelativistic beam (for example, the blue lines for $\gamma=1.2$ ) is smaller than that for a relativistic beam at high frequency (in the longitudinal case) or at any frequency (in the transverse case). The physical reason for this behavior is as follows. The nonrelativistic (slower) beam receives more fluctuating kicks from the wakefields while going through them. These kicks tend to cancel each other in net, resulting in the diminishing impedance. However, longitudinal wakes are excited by the beam like cosine function [5] and they are nearly constant at low frequency during the beam passage. Therefore, the longitudinal impedance has little dependence on the Lorentz- $\gamma$ factor at low frequency (the transverse impedance is roughly proportional to Lorentz- $\beta$ factor).

\section{SUMMARY}

A new theory is developed to describe longitudinal and transverse impedances of ceramic breaks both with and without the TiN coating. The theoretical and numerical simulation results calculated by ABCI and CST Studio are all in good agreement for the ceramic break without the TiN coating. Particularly, the agreements between the theoretical and ABCI's results are excellent, while CST Studio tends to provide higher impedances, notably at resonance frequencies, than the others.

The impedance of the ceramic break has resonance structures, because the difference of the dielectric constants between ceramic and the vacuum introduces the reflection of waves at their boundary, and this reflection of electromagnetic fields at the lower and the upper boundaries of the ceramic creates trapped modes inside. The resonances can be categorized into the cavity-mode resonances and the coaxial-mode resonances.

Despite the existence of the resonances, the loss factor of the ceramic is a few times smaller than that of a vacuum gap, due to the wavelength contraction effect inside the ceramic. In other words, the filling of the gap by the ceramic helps to reduce the radiation loss of a beam in most cases, in particular, for a long bunch.

Generally speaking, the impedance becomes smaller as the velocity of a nonrelativistic beam decreases, since a slower beam receives more fluctuating kicks from wakefields while passing through them. In fact, we find that the transverse impedance is roughly proportional to the Lorentz- $\beta$ factor at any frequency. On the other hand, the longitudinal impedance depends on the Lorentz- $\gamma$ factor only weakly at low frequency, since slowly oscillating wakefields provide similar kicks to a beam regardless of its velocity.

The theory can be applied to the calculation of the impedance of a ceramic break whose inner surface is coated with thin TiN (the outer surface is just exposed to vacuum). When the TiN coating is thick (larger than $t_{\min }$ ), the entire image current runs in the TiN coating, which shields wakefields inside the chamber from reaching out to the ceramic. In this case, the existence of ceramic has no effect on the impedance. Consequently, the impedance becomes higher as the TiN becomes thinner.

When a conductive wall is added to the outer surface, the behavior of the impedance is quite changed. In this case, the perfectly conductive wall makes the impedance go to zero at zero frequency. Moreover, the longitudinal impedance takes the maximum at a certain thickness. Typically, for the case studied here, this worst thickness is of the order of several $\mu \mathrm{m}$, which may be too thick to realize in many machines. Below this thickness, thinner coating is preferable from the impedance point of view. 
In a real machine, the image current may find a different path from the one between the gap or in a thin TiN coating, if it provides a smaller impedance and less energy loss to a beam. Several bench measurements have been done for the evaluation of impedance of isolated gaps equipped with one or more bypass units [21]. It would be interesting that our theory for the ceramic break with the TiN coating is benchmarked with those measurements.

\section{ACKNOWLEDGMENTS}

The authors would like to thank T. Toyama. The authors also would like to thank all members of J-PARC project at JAEA/KEK.

\section{APPENDIX A: DERIVATION OF FORMULAS OF IMPEDANCES OF CERAMIC BREAKS}

\section{Longitudinal impedances}

When a beam has a cylindrically uniform density with radius $\sigma$ and its total charge is $1 \mathrm{C} / \mathrm{m}$, its current density is given by Eq. (1) in the text.

The formal solutions of the fields inside the chamber at the angular frequency $\omega$ are given by

$$
\begin{aligned}
E_{z}= & \frac{j k}{\gamma^{2}} \frac{c Z_{0}}{\pi \sigma^{2}}\left(\frac{1}{\bar{k}^{2}}-\frac{\sigma I_{0}(\bar{k} \rho) K_{1}(\bar{k} \sigma)}{\bar{k}}\right) e^{-j k z} \\
& +\int_{-\infty}^{\infty} d h \mathcal{A}_{0}(h) e^{-j h z} \frac{J_{0}(\Lambda \rho)}{J_{0}(\Lambda a)}, \\
H_{\theta}= & \frac{\beta c}{\pi \sigma} K_{1}(\bar{k} \sigma) I_{1}(\bar{k} \rho) e^{-j k z} \\
& +\frac{j k \beta}{Z_{0}} \int_{-\infty}^{\infty} d h \mathcal{A}_{0}(h) e^{-j h z} \frac{J_{1}(\Lambda \rho)}{\Lambda J_{0}(\Lambda a)},
\end{aligned}
$$

for $\rho<\sigma$, and

$$
\begin{gathered}
E_{z}=\frac{j c Z_{0}}{\pi \sigma \gamma} I_{1}(\bar{k} \sigma) K_{0}(\bar{k} \rho) e^{-j k z} \\
\quad+\int_{-\infty}^{\infty} d h \mathcal{A}_{0}(h) e^{-j h z} \frac{J_{0}(\Lambda \rho)}{J_{0}(\Lambda a)}, \\
H_{\theta}=\frac{\beta c}{\pi \sigma} I_{1}(\bar{k} \sigma) K_{1}(\bar{k} \rho) e^{-j k z} \\
+\frac{j k \beta}{Z_{0}} \int_{-\infty}^{\infty} d h \mathcal{A}_{0}(h) e^{-j h z} \frac{J_{1}(\Lambda \rho)}{\Lambda J_{0}(\Lambda a)},
\end{gathered}
$$

for $\rho>\sigma$, where cylindrical coordinates $(\rho, \theta, z)$ are used, $\Lambda=\sqrt{k^{2} \beta^{2}-h^{2}}, c$ is the velocity of light, $\beta=v / c, v$ is the velocity of the beam, $k=\omega / c \beta, Z_{0}(=120 \pi \Omega)$ is the impedance of free space, $J_{n}(z)$ is the Bessel function, $I_{n}(z)$ and $K_{n}(z)$ are the modified Bessel functions [13], $\gamma$ is Lorentz- $\gamma, \bar{k}=k / \gamma$ and $\mathcal{A}_{0}(h)$ is an expansion coefficient. The time dependence of the fields is assumed to be harmonic and it is expressed as the complex exponential $e^{j \omega t}$

Since $E_{z}$ on the inner surface of the perfectly conductive chambers that sandwich the ceramic break should be zero (refer to Fig. 1), the expansion coefficient $\mathcal{A}_{0}(h)$ should satisfy the following relation:

$$
\begin{aligned}
& \frac{j c Z_{0}}{\pi \sigma \gamma} I_{1}(\bar{k} \sigma) K_{0}(\bar{k} a) e^{-j k z}+\int_{-\infty}^{\infty} d h \mathcal{A}_{0}(h) e^{-j h z} \\
& \quad= \begin{cases}\frac{V_{1}}{2 w}+\frac{\sum_{m=1}^{\infty} V_{1}^{(m)} \cos \frac{m \pi(z+w)}{2 w}}{w} & \text { for }-w<z<w \\
0 & \text { otherwise, }\end{cases}
\end{aligned}
$$

where $V_{1}$ is the voltage on the inner surface of the ceramic break at $\rho=a$, and $V_{1}^{(m)}$ are expansion coefficients. Consequently, the original expansion coefficient $\mathcal{A}_{0}(h)$ is rewritten by using the new expansion coefficients $V_{1}$ and $V_{1}^{(m)}$ as

$$
\begin{aligned}
& \frac{j c Z_{0}}{\pi \sigma \gamma} I_{1}(\bar{k} \sigma) K_{0}(\bar{k} a) \delta(h-k)+\mathcal{A}_{0}(h) \\
& \quad=\frac{V_{1}}{2 \pi} \frac{\sin h w}{h w}+\sum_{m=1}^{\infty} \frac{V_{1}^{(m)}\left[(-1)^{m} e^{j h w}-e^{-j h w}\right] h}{j 2 \pi w\left(h^{2}-\frac{m^{2} \pi^{2}}{4 w^{2}}\right)},
\end{aligned}
$$

where $g=2 w$ is the gap size and $\delta(z)$ is the $\delta$ function. Substituting Eq. (A6) into Eqs. (A1)-(A4), we obtain Eqs. (2)-(5) in the text.

For the fields outside the ceramic break, Silver and Saunders's theory [22] gives their descriptions as

$$
\begin{aligned}
E_{z}= & \frac{V_{2}}{2 \pi} \int_{-\infty}^{\infty} d h \frac{H_{0}^{(2)}(\Lambda \rho)}{H_{0}^{(2)}\left(\Lambda a_{2}\right)} \frac{\sin h w}{h w} e^{-j h z} \\
& -\sum_{m=1}^{\infty} V_{2}^{(m)} \int_{-\infty}^{\infty} d h e^{-j h z} \frac{j h\left[(-1)^{m} e^{j h w}-e^{-j h w}\right]}{2 \pi w\left(h^{2}-\frac{m^{2} \pi^{2}}{4 w^{2}}\right)} \\
& \times \frac{H_{0}^{(2)}(\Lambda \rho)}{H_{0}^{(2)}\left(\Lambda a_{2}\right)} \\
H_{\theta}= & j \frac{\beta k}{Z_{0}} \frac{V_{2}}{2 \pi} \int_{-\infty}^{\infty} d h \frac{H_{1}^{(2)}(\Lambda \rho)}{\Lambda H_{0}^{(2)}\left(\Lambda a_{2}\right)} \frac{\sin h w}{h w} e^{-j h z} \\
& -\sum_{m=1}^{\infty} V_{2}^{(m)} \frac{k \beta}{Z_{0} w} \int_{-\infty}^{\infty} d h e^{-j h z} \\
& \times \frac{h\left[(-1)^{m} e^{j h w}-e^{-j h w}\right]}{2 \pi\left(h^{2}-\frac{m^{2} \pi^{2}}{4 w^{2}}\right)} \frac{H_{0}^{(2)}(\Lambda \rho)}{\Lambda H_{0}^{(2)}\left(\Lambda a_{2}\right)}
\end{aligned}
$$

for $\rho>a_{2}$ (the outer size of the ceramic break), where $H_{m}^{(2)}(z)$ is the Hankel functions [13] of the second kind, the prime means the differential by its argument $z$, and $V_{2}$ is the voltage on the outer surface of the ceramic break at $\rho=a_{2}$, and $V_{2}^{(m)}$ are the expansion coefficients. Here we should 
notice that Eq. (A7) satisfies the condition that $E_{z}$ should be zero on the outer surface of the chamber except on the ceramic break.

Before the fields on the inner and the outer surface of the ceramic break are associated, let us expand the fields on the respective surfaces by sinusoidal functions. Because the ceramic is sandwiched by the perfectly conductive chambers, the fields in the ceramic $(-w<z<w)$ on $\rho=a$ and $\rho=a_{2}$ may be expressed as

$$
\begin{gathered}
E_{z}(a)=\frac{V_{1}}{2 w}+\frac{\sum_{m=1}^{\infty} V_{1}^{(m)} \cos \frac{m \pi(z+w)}{2 w}}{w}, \\
H_{\theta}(a)=\alpha(z)+\frac{j k \beta}{2 \pi Z_{0}} V_{1} J(z)+\frac{k \beta}{2 \pi w Z_{0}} \sum_{m=1}^{\infty} V_{1}^{(m)} J^{(m)}(z) \\
=\langle\alpha(z)\rangle+\frac{j k \beta}{2 \pi Z_{0}} V_{1}\langle J(z)\rangle+\frac{k \beta}{2 \pi w Z_{0}} \sum_{m=1}^{\infty} V_{1}^{(m)}\left\langle J^{(m)}(z)\right\rangle \\
+\frac{1}{w} \sum_{m=1}^{\infty} \cos \frac{m \pi(z+w)}{2 w}[\langle\alpha(z)\rangle\rangle_{m}+\frac{j k \beta}{2 \pi Z_{0}} V_{1}\langle\langle J(z)\rangle\rangle_{m}+\frac{k \beta}{2 \pi w Z_{0}} \sum_{n=1}^{\infty} V_{1}^{(n)}\left\langle\left\langle J^{(n)}(z)\right\rangle_{m}\right],
\end{gathered}
$$

and

$$
\begin{gathered}
E_{z}\left(a_{2}\right)=\frac{V_{2}}{2 w}+\frac{\sum_{m=1}^{\infty} V_{2}^{(m)} \cos \frac{m \pi(z+w)}{2 w}}{w}, \\
H_{\theta}\left(a_{2}\right)=-j \frac{\beta k}{Z_{0}} \frac{V_{2}}{2 \pi} Y(z)-\sum_{m=1}^{\infty} V_{2}^{(m)} \frac{k \beta}{2 \pi Z_{0} w} Y^{(m)}(z) \\
=-j \frac{\beta k}{Z_{0}} \frac{V_{2}}{2 \pi}\langle Y(z)\rangle-\sum_{m=1}^{\infty} V_{2}^{(m)} \frac{k \beta}{2 \pi Z_{0} w}\left\langle Y^{(m)}(z)\right\rangle \\
+\frac{1}{w} \sum_{m=1}^{\infty} \cos \frac{m \pi(z+w)}{2 w}\left[-j \frac{\beta k}{Z_{0}} \frac{V_{2}}{2 \pi}\left\langle\langle Y(z)\rangle_{m}-\frac{k \beta}{2 \pi Z_{0} w} \sum_{n=1}^{\infty} V_{2}^{(n)}\left\langle\left\langle Y^{(n)}(z)\right\rangle\right\rangle_{m}\right],\right.
\end{gathered}
$$

respectively, where

$$
\begin{aligned}
& \alpha(z)=\frac{\beta c I_{1}(\bar{k} \sigma)}{\pi \sigma \bar{k} a I_{0}(\bar{k} a)} e^{-j k z}, \\
& J(z)=\int_{-\infty}^{\infty} e^{-j h z} \frac{J_{1}(\Lambda a)}{\Lambda J_{0}(\Lambda a)} \frac{\sin h w}{h w} d h \\
& =-\sum_{s=1}^{\infty} \frac{\pi a\left(2-e^{-j \sqrt{k^{2} \beta^{2}-\frac{j_{0, s}^{2}}{a^{2}}}(z+w)}-e^{j \sqrt{k^{2} \beta^{2}-\frac{j_{0, s}^{2}}{a^{2}}}(z-w)}\right)}{w\left(k^{2} \beta^{2} a^{2}-j_{0, s}^{2}\right)}, \\
& J^{(m)}(z)=\int_{-\infty}^{\infty} e^{-j h z} \frac{J_{1}(\Lambda a)}{\Lambda J_{0}(\Lambda a)} \frac{\left[(-1)^{m} e^{j h w}-e^{-j h w}\right] h}{\left(h^{2}-\frac{m^{2} \pi^{2}}{4 w^{2}}\right)} d h, \\
& Y(z)=-\int_{-\infty}^{\infty} \frac{H_{1}^{(2)}\left(\Lambda a_{2}\right)}{\Lambda H_{0}^{(2)}\left(\Lambda a_{2}\right)} \frac{\sin h w}{h w} e^{-j h z} d h,
\end{aligned}
$$$$
Y^{(m)}(z)=\int_{-\infty}^{\infty} e^{-j h z} \frac{h\left[(-1)^{m} e^{j h w}-e^{-j h w}\right]}{\left(h^{2}-\frac{m^{2} \pi^{2}}{4 w^{2}}\right)} \frac{H_{0}^{\prime(2)}\left(\Lambda a_{2}\right)}{\Lambda H_{0}^{(2)}\left(\Lambda a_{2}\right)} d h .
$$

The brackets in Eqs. (A10) and (A12) are defined as

$$
\begin{gathered}
\langle\cdots\rangle \equiv \frac{1}{2 w} \int_{-w}^{w} d z \cdots \\
\left\langle\langle\cdots\rangle_{m} \equiv \int_{-w}^{w} d z \cos \frac{m \pi(z+w)}{2 w} \cdots\right.
\end{gathered}
$$

The concrete expressions for $\langle\alpha\rangle,\langle J\rangle,\left\langle J^{(m)}\right\rangle,\langle\langle\alpha\rangle\rangle_{m},\left\langle\langle J\rangle_{m}\right.$, $\left\langle\left\langle J^{(n)}\right\rangle\right\rangle_{m},\langle Y\rangle_{m},\left\langle Y^{(m)}\right\rangle,\left\langle\langle Y\rangle_{m}\right.$ and $\left\langle\left\langle Y^{(n)}\right\rangle\right\rangle_{m}$ are given in Appendix B.

The solutions of Maxwell equations in the ceramic transfer the fields on $\rho=a$ to those on $\rho=a_{2}$ (refer to Appendix C). The expansion coefficients $V_{1}, V_{1}^{(m)}, V_{2}$ and $V_{2}^{(m)}$ are determined by the connection conditions of $E_{z}$ and $H_{\theta}$ on $\rho=a_{2}$. Consequently, we obtain the relations 


$$
\begin{gathered}
\frac{V_{2}}{2 w}=A_{0}^{(0)} \frac{V_{1}}{2 w}+C_{0}^{(0)}\left(\langle\alpha(z)\rangle+\frac{V_{1}}{2 \pi} \frac{j k \beta}{Z_{0}}\langle J(z)\rangle+\frac{k \beta}{2 \pi w Z_{0}} \sum_{m=1}^{\infty} V_{1}^{(m)}\left\langle J^{(m)}(z)\right\rangle\right), \\
0=I_{0}^{(0)} \frac{V_{1}}{2 w}+K_{0}^{(0)}\left(\langle\alpha(z)\rangle+\frac{V_{1}}{2 \pi} \frac{j k \beta}{Z_{0}}\langle J(z)\rangle+\frac{k \beta}{2 \pi w Z_{0}} \sum_{m=1}^{\infty} V_{1}^{(m)}\left\langle J^{(m)}(z)\right\rangle\right) \\
\quad+\frac{j k \beta V_{2}}{2 \pi Z_{0}}\langle Y(z)\rangle+\sum_{m=1}^{\infty} V_{2}^{(m)} \frac{k \beta}{2 \pi Z_{0} w}\left\langle Y^{(m)}(z)\right\rangle, \\
V_{2}^{(m)}=A_{0}^{(m)} V_{1}^{(m)}+C_{0}^{(m)}\left(\langle\langle\alpha(z)\rangle\rangle_{m}+\frac{j k \beta}{2 \pi Z_{0}} V_{1}\langle\langle J(z)\rangle\rangle_{m}+\frac{k \beta}{2 \pi w Z_{0}} \sum_{n=1}^{\infty} V_{1}^{(n)}\left\langle\left\langle J^{(n)}(z)\right\rangle\right\rangle_{m}\right), \\
-j \frac{\beta k}{Z_{0}} \frac{V_{2}}{2 \pi}\langle\langle Y(z)\rangle\rangle_{m}-\frac{k \beta}{2 \pi Z_{0} w} \sum_{n=1}^{\infty} V_{2}^{(n)}\left\langle\left\langle Y^{(n)}(z)\right\rangle\right\rangle_{m} \\
=I_{0}^{(m)} V_{1}^{(m)}+K_{0}^{(m)}\left(\langle\langle\alpha(z)\rangle\rangle_{m}+\frac{j k \beta}{2 \pi Z_{0}} V_{1}\langle\langle J(z)\rangle\rangle_{m}+\frac{k \beta}{2 \pi w Z_{0}} \sum_{n=1}^{\infty} V_{1}^{(n)}\left\langle\left\langle J^{(n)}(z)\right\rangle_{m}\right),\right.
\end{gathered}
$$

where $A_{0}^{(0)}, C_{0}^{(0)}, I_{0}^{(0)}, K_{0}^{(0)}, A_{0}^{(m)}, C_{0}^{(m)}, I_{0}^{(m)}$ and $K_{0}^{(m)}$ with positive integer $m$, which are determined by the solutions of Maxwell equations, are the coefficients that transfer the fields on the inner surface $(\rho=a)$ to the outer surface $\left(\rho=a_{2}\right)$ of the ceramic break. Concrete expressions of the transfer coefficients are calculated in Appendix C 1. All expansion coefficients $V_{1}, V_{1}^{(m)}, V_{2}$ and $V_{2}^{(m)}$ are obtained by solving Eqs. (A20)-(A23) up to any order of $m$.

The average value of $E_{z}$ [expressed by Eq. (A1)] over $\rho$ gives the longitudinal impedance $Z_{L}$. Finally, it can be expressed as Eq. (6) in the text.

\section{Transverse impedance}

In order to calculate the transverse impedance, let us consider a beam with the charge distribution of the azimuthal dependence as $j_{z}=q \beta c \delta\left(\rho-r_{b}\right) \cos \theta e^{-j k z} / \pi r_{b}$. When the beam is traveling in the chamber, the fields inside the chamber are

$$
E_{z}=i_{1}\left[\frac{j k}{\gamma^{2}} \frac{c Z_{0}}{\pi}\left(K_{1}\left(\bar{k} r_{b}\right)-K_{1}(\bar{k} a) \frac{I_{1}\left(\bar{k} r_{b}\right)}{I_{1}(\bar{k} a)}\right) I_{1}(\bar{k} \rho) \frac{e^{-j k z}}{r_{b}}+\int_{-\infty}^{\infty} d h e^{-j h z} \mathcal{A}_{1}(h) \frac{J_{1}(\Lambda \rho)}{J_{1}(\Lambda a)}\right] \cos \theta
$$

for $\rho<r_{b}$ and

$$
\begin{gathered}
E_{z}=i_{1}\left[\frac{j k}{\gamma^{2}} \frac{c Z_{0}}{\pi} K_{1}(\bar{k} \rho) I_{1}\left(\bar{k} r_{b}\right) \frac{e^{-j k z}}{r_{b}}+\int_{-\infty}^{\infty} d h \mathcal{A}_{1}(h) e^{-j h z} \frac{J_{1}(\Lambda \rho)}{J_{1}(\Lambda a)}\right] \cos \theta \\
H_{\theta}=i_{1}\left[c \beta \frac{\bar{k}}{2 \pi}\left[K_{0}(\bar{k} \rho)+K_{2}(\bar{k} \rho)\right] I_{1}\left(\bar{k} r_{b}\right) \frac{e^{-j k z}}{r_{b}}-\int_{-\infty}^{\infty} d h e^{-j h z} \frac{j h}{\Lambda^{2}}\left(\frac{\mathcal{B}_{1}(h) J_{1}(\Lambda \rho)}{\rho J_{1}(\Lambda a)}+\frac{k \beta \Lambda}{Z_{0} h} \mathcal{A}_{1}(h) \frac{J_{1}^{\prime}(\Lambda \rho)}{J_{1}(\Lambda a)}\right)\right] \cos \theta \\
H_{z}=i_{1} \int_{-\infty}^{\infty} d h \mathcal{B}_{1}(h) e^{-j h z} \frac{J_{1}(\Lambda \rho)}{J_{1}(\Lambda a)} \sin \theta \\
E_{\theta}=i_{1}\left[\frac{c Z_{0}}{\pi \rho} K_{1}(\bar{k} \rho) I_{1}\left(\bar{k} r_{b}\right) \frac{e^{-j k z}}{r_{b}}\right. \\
\left.+\int_{-\infty}^{\infty} d h e^{-j h z} \frac{j k \beta Z_{0}}{\Lambda^{2}}\left(\mathcal{B}_{1}(h) \Lambda \frac{J_{1}^{\prime}(\Lambda \rho)}{J_{1}(\Lambda a)}+\frac{h}{Z_{0} k \beta \rho} \mathcal{A}_{1}(h) \frac{J_{1}(\Lambda \rho)}{J_{1}(\Lambda a)}\right)\right] \sin \theta
\end{gathered}
$$

for $\rho>r_{b}$, where $i_{1}=q r_{b}, \mathcal{A}_{1}(h)$ and $\mathcal{B}_{1}(h)$ are expansion coefficients. Since $E_{z}$ and $E_{\theta}$ on the inner surface of the chamber should be zero except on the ceramic, the expansion coefficients $\mathcal{A}_{1}(h)$ and $\mathcal{B}_{1}(h)$ should satisfy the following relations: 


$$
\begin{gathered}
\frac{j k}{\gamma^{2}} \frac{c Z_{0}}{\pi} K_{1}(\bar{k} a) I_{1}\left(\bar{k} r_{b}\right) \frac{e^{-j k z}}{r_{b}}+\int_{-\infty}^{\infty} d h \mathcal{A}_{1}(h) e^{-j h z}= \begin{cases}\frac{\pi \tilde{\mathcal{V}}_{2}}{w}+\sum_{m=1}^{\infty} \frac{\pi \tilde{\mathcal{V}}_{2}^{(m)} \cos \frac{m \pi(z+w)}{2 w}}{w} & \text { for }-w<z<w \\
0 & \text { otherwise }\end{cases} \\
\frac{c Z_{0}}{\pi a} K_{1}(\bar{k} a) I_{1}\left(\bar{k} r_{b}\right) \frac{e^{-j k z}}{r_{b}}+\int_{-\infty}^{\infty} d h e^{-j h z} \frac{j k \beta Z_{0}}{\Lambda^{2}}\left(\mathcal{B}_{1}(h) \Lambda \frac{J_{1}^{\prime}(\Lambda a)}{J_{1}(\Lambda a)}+\frac{h}{Z_{0} k \beta a} \mathcal{A}_{1}(h)\right) \\
= \begin{cases}\sum_{m=1}^{\infty} \frac{\pi \tilde{\mathcal{V}}_{1}^{(m)} \sin \frac{m \pi(z+w)}{2 w}}{w} & \text { for }-w<z<w \\
0 & \text { otherwise, }\end{cases}
\end{gathered}
$$

where $\tilde{\mathcal{V}}_{2}$ is the voltage on the inner surface of the ceramic at $\rho=a$, and $\tilde{\mathcal{V}}_{1}^{(m)}$ and $\tilde{\mathcal{V}}_{2}^{(m)}$ are the expansion coefficients. Consequently, the expansion coefficients $\mathcal{A}_{1}(h)$ and $\mathcal{B}_{1}(h)$ are rewritten by using $\tilde{\mathcal{V}}_{1}^{(m)}, \tilde{\mathcal{V}}_{2}^{(m)}$ and $\tilde{\mathcal{V}}_{2}$ as

$$
\begin{gathered}
\frac{j k}{\gamma^{2}} \frac{c Z_{0}}{\pi} K_{1}(\bar{k} a) I_{1}\left(\bar{k} r_{b}\right) \frac{\delta(h-k)}{r_{b}}+\mathcal{A}_{1}(h)=\tilde{\mathcal{V}}_{2} \frac{\sin h w}{h w}+\sum_{m=1}^{\infty} \tilde{\mathcal{V}}_{2}^{(m)} \frac{h\left[(-1)^{m} e^{j h w}-e^{-j h w}\right]}{j 2 w\left(h^{2}-\frac{m^{2} \pi^{2}}{4 w^{2}}\right)} \\
\frac{c Z_{0}}{\pi a} K_{1}(\bar{k} a) I_{1}\left(\bar{k} r_{b}\right) \frac{\delta(h-k)}{r_{b}}+\frac{j k \beta Z_{0}}{\Lambda^{2}}\left(\mathcal{B}_{1}(h) \Lambda \frac{J_{1}^{\prime}(\Lambda a)}{J_{1}(\Lambda a)}+\frac{h}{Z_{0} k \beta a} \mathcal{A}_{1}(h)\right)=\sum_{m=1}^{\infty} \tilde{\mathcal{V}}_{1}^{(m)} \frac{m \pi\left[(-1)^{m} e^{j h w}-e^{-j h w}\right]}{4 w^{2}\left(h^{2}-\frac{m^{2} \pi^{2}}{4 w^{2}}\right)} .
\end{gathered}
$$

Substituting Eqs. (A31) and (A32) into Eqs. (A24)-(A28), we obtain Eqs. (12)-(16) in the text.

The fields outside the chamber are given by

$$
\begin{aligned}
& E_{z}=i_{1}\left[\mathcal{V}_{2} \int_{-\infty}^{\infty} d h e^{-j h z} \frac{\sin (h w)}{h w} \frac{H_{1}^{(2)}(\Lambda \rho)}{H_{1}^{(2)}\left(\Lambda a_{2}\right)}-\sum_{m=1}^{\infty} \mathcal{V}_{2}^{(m)} \int_{-\infty}^{\infty} d h e^{-j h z} \frac{j h\left[(-1)^{m} e^{j h w}-e^{-j h w}\right]}{2 w\left(h^{2}-\frac{m^{2} \pi^{2}}{4 w^{2}}\right)} \frac{H_{1}^{(2)}(\Lambda \rho)}{H_{1}^{(2)}\left(\Lambda a_{2}\right)}\right] \cos \theta \\
& H_{\theta}=-i_{1} \mathcal{V}_{2} \frac{j k \beta}{Z_{0}}\left[\int_{-\infty}^{\infty} d h e^{-j h z} \frac{\sin (h w)}{h w} \frac{H_{1}^{\prime(2)}(\Lambda \rho)}{\Lambda H_{1}^{(2)}\left(\Lambda a_{2}\right)}-\frac{1}{k^{2} \beta^{2} a_{2} \rho} \int_{-\infty}^{\infty} d h e^{-j h z} \frac{\sin (h w)}{h w} \frac{h^{2} H_{1}^{(2)}(\Lambda \rho)}{\Lambda^{3} H_{1}^{\prime(2)}\left(\Lambda a_{2}\right)}\right] \cos \theta \\
& -i_{1} \sum_{m=1}^{\infty} \mathcal{V}_{2}^{(m)} \frac{k \beta}{Z_{0} w}\left[\int_{-\infty}^{\infty} d h e^{-j h z} \frac{h\left[(-1)^{m} e^{j h w}-e^{-j h w}\right]}{2\left(h^{2}-\frac{m^{2} \pi^{2}}{4 w^{2}}\right)} \frac{H_{1}^{\prime(2)}(\Lambda \rho)}{\Lambda H_{1}^{(2)}\left(\Lambda a_{2}\right)}\right. \\
& \left.-\frac{1}{2 k^{2} \beta^{2} a_{2} \rho} \int_{-\infty}^{\infty} d h e^{-j h z} \frac{h^{3}\left[(-1)^{m} e^{j h w}-e^{-j h w}\right] H_{1}^{(2)}(\Lambda \rho)}{\left(h^{2}-\frac{m^{2} \pi^{2}}{4 w^{2}}\right) \Lambda^{3} H_{1}^{\prime(2)}\left(\Lambda a_{2}\right)}\right] \cos \theta \\
& -i_{1} \sum_{m=1}^{\infty} \mathcal{V}_{1}^{(m)} \frac{m \pi}{4 k \beta w^{2} Z_{0} \rho} \int_{-\infty}^{\infty} d h e^{-j h z} \frac{h H_{1}^{(2)}(\Lambda \rho)\left[(-1)^{m} e^{j h w}-e^{-j h w}\right]}{\Lambda\left(h^{2}-\frac{m^{2} \pi^{2}}{4 w^{2}}\right) H_{1}^{\prime(2)}\left(\Lambda a_{2}\right)} \cos \theta, \\
& H_{z}=-i_{1} \mathcal{V}_{2} \int_{-\infty}^{\infty} d h e^{-j h z} \frac{\sin (h w)}{h w} \frac{h H_{1}^{(2)}(\Lambda \rho)}{k \beta Z_{0} a_{2} \Lambda H_{1}^{(2)}\left(\Lambda a_{2}\right)} \\
& +i_{1} \sum_{m=1}^{\infty} \mathcal{V}_{2}^{(m)} \frac{j}{2 k \beta Z_{0} a_{2} w} \int_{-\infty}^{\infty} d h e^{-j h z} \frac{h^{2}\left[(-1)^{m} e^{j h w}-e^{-j h w}\right]}{\left(h^{2}-\frac{m^{2} \pi^{2}}{4 w^{2}}\right)} \frac{H_{1}^{(2)}(\Lambda \rho)}{\Lambda H_{1}^{\prime(2)}\left(\Lambda a_{2}\right)} \sin \theta \\
& -i_{1} \sum_{m=1}^{\infty} \mathcal{V}_{1}^{(m)} \frac{j m \pi}{4 k \beta w^{2} Z_{0}} \int_{-\infty}^{\infty} d h e^{-j h z} \frac{\Lambda\left[(-1)^{m} e^{j h w}-e^{-j h w}\right] H_{1}^{(2)}(\Lambda \rho)}{\left(h^{2}-\frac{m^{2} \pi^{2}}{4 w^{2}}\right) H_{1}^{(2)}\left(\Lambda a_{2}\right)} \sin \theta, \\
& E_{\theta}=-i_{1} \mathcal{V}_{2} \int_{-\infty}^{\infty} d h e^{-j h z} \frac{\sin (h w)}{h w} \frac{j h H_{1}^{(2)}(\Lambda \rho)}{a_{2} \Lambda^{2} H_{1}^{\prime(2)}\left(\Lambda a_{2}\right)} \sin \theta+i_{1} \mathcal{V}_{2} \int_{-\infty}^{\infty} d h e^{-j h z} \frac{\sin (h w)}{h w} \frac{j h H_{1}^{(2)}(\Lambda \rho)}{\rho \Lambda^{2} H_{1}^{(2)}\left(\Lambda a_{2}\right)} \sin \theta \\
& +i_{1} \sum_{m=1}^{\infty} \mathcal{V}_{1}^{(m)} \int_{-\infty}^{\infty} d h \frac{H_{1}^{(2)}(\Lambda \rho)}{H_{1}^{\prime(2)}\left(\Lambda a_{2}\right)} \frac{m \pi\left[(-1)^{m} e^{j h w}-e^{-j h w}\right]}{4 w^{2}\left(h^{2}-\frac{m^{2} \pi^{2}}{4 w^{2}}\right)} e^{-j h z} \sin \theta
\end{aligned}
$$

where $\mathcal{V}_{2}$ is the voltage on the outer surface of the ceramic at $\rho=a_{2}$, and $\mathcal{V}_{1}^{(m)}$ and $\mathcal{V}_{2}^{(m)}$ are the expansion coefficients. 
On the inner surface of the ceramic $(\rho=a, \theta,-w<z<w)$, Eqs. (13)-(16) in the text are expanded by sinusoidal functions as

$$
E_{z}(a)=\tilde{E}_{z}(a) \cos \theta=\left(i_{1} \frac{\pi \tilde{\mathcal{V}}_{2}}{w}+i_{1} \sum_{m=1}^{\infty} \frac{\cos \frac{m \pi(z+w)}{2 w}}{w} \pi \tilde{\mathcal{V}}_{2}^{(m)}\right) \cos \theta
$$

$$
\begin{aligned}
H_{\theta}(a)= & \tilde{H}_{\theta}(a) \cos \theta \\
= & i_{1}\left\langle\frac{c \beta I_{1}\left(\bar{k} r_{b}\right) e^{-j k z}}{r_{b} \pi a I_{1}(\bar{k} a)}-\frac{j k \beta}{Z_{0}}\left(Y_{2}(z)-\frac{Y_{3}(z)}{k^{2} \beta^{2} a^{2}}\right) \tilde{\mathcal{V}}_{2}\right\rangle \cos \theta \\
& -i_{1} \sum_{n=1}^{\infty} \tilde{\mathcal{V}}_{1}^{(n)} \frac{n \pi}{2 w^{2} Z_{0} k \beta a}\left\langle Y_{4}^{\prime(n)}(z)\right\rangle \cos \theta-i_{1} \sum_{n=1}^{\infty} \tilde{\mathcal{V}}_{2}^{(n)} \frac{k \beta}{w Z_{0}}\left\langle Y_{1}^{(n)}(z)-\frac{Y_{4}^{(n)}(z)}{k^{2} \beta^{2} a^{2}}\right\rangle \cos \theta \\
& +i_{1} \sum_{m=1}^{\infty} \frac{\cos \left(\frac{m \pi(z+w)}{2 w}\right)}{w}\left[\frac{j c \beta k I_{1}\left(\bar{k} r_{b}\right)\left[e^{j k w}-(-1)^{m} e^{-j k w}\right]}{r_{b} \pi a I_{1}(\bar{k} a)\left(\frac{m^{2} \pi^{2}}{4 w^{2}}-k^{2}\right)}\right. \\
& -\frac{j k \beta}{Z_{0}}\left\langle\left\langle Y_{2}(z)-\frac{Y_{3}(z)}{k^{2} \beta^{2} a^{2}}\right\rangle \tilde{\mathcal{V}}_{m}-\sum_{n=1}^{\infty} \frac{k \beta}{w Z_{0}}\left\langle\left\langle Y_{1}^{(n)}(z)-\frac{Y_{4}^{(n)}(z)}{k^{2} \beta^{2} a^{2}}\right\rangle\right\rangle_{m} \tilde{\mathcal{V}}_{2}^{(n)}-\sum_{n=1}^{\infty} \frac{n \pi \tilde{\mathcal{V}}_{1}^{(n)}}{2 w^{2} k \beta a Z_{0}}\left\langle\left\langle Y_{4}^{\prime(n)}\right\rangle\right\rangle_{m}\right] \cos \theta, \\
H_{z}(a)= & i_{1}\left[\sum_{n=1}^{\infty} \tilde{\mathcal{V}}_{1}^{(n)} \frac{n \pi}{2 w^{2} j k \beta Z_{0}}\left\langle Y_{-2}^{(n)}\right\rangle-\frac{\tilde{\mathcal{V}}_{2}}{a k \beta Z_{0}}\left\langle Y_{-1}^{(n)}(z)\right\rangle-\sum_{n=1}^{\infty} \frac{\tilde{\mathcal{V}}_{2}^{(n)}}{j w a k \beta Z_{0}}\left\langle Y_{0}^{(n)}\right\rangle\right] \sin \theta \\
& +i_{1} \sum_{m=1}^{\infty} \frac{\sin \left(\frac{m \pi(z+w)}{2 w}\right)}{w}\left[-\sum_{n=1}^{\infty} \frac{\tilde{\mathcal{V}}_{2}^{(n)}}{j w a k \beta Z_{0}}\left\langle\left\langle Y_{0}^{(n)}(z)\right\rangle\right\rangle_{m}^{s}-\frac{\tilde{\mathcal{V}}_{2}}{a k \beta Z_{0}}\left\langle\left\langle Y_{-1}(z)\right\rangle\right\rangle_{m}^{s}+\sum_{n=1}^{\infty} \frac{n \pi j \tilde{\mathcal{V}}_{1}^{(n)}}{2 j k \beta Z_{0} w^{2}}\left\langle\left\langle Y_{-2}^{(n)}\right\rangle\right\rangle_{m}^{s}\right] \sin \theta,
\end{aligned}
$$

$$
E_{\theta}(a)=i_{1} \sum_{m=1}^{\infty} \frac{\sin \frac{m \pi(z+w)}{2 w}}{w} \pi \tilde{\mathcal{V}}_{1}^{(m)} \sin \theta
$$

where

$$
\begin{gathered}
Y_{-2}^{(n)}(z)=\int_{-\infty}^{\infty} d h e^{-j h z} \frac{\Lambda\left[(-1)^{n} e^{j h w}-e^{-j h w}\right]}{2\left(h^{2}-\frac{n^{2} \pi^{2}}{4 w^{2}}\right)} \frac{J_{1}(\Lambda a)}{J_{1}^{\prime}(\Lambda a)} \\
Y_{-1}(z)=\int_{-\infty}^{\infty} d h e^{-j h z} \frac{\sin h w}{h w} \frac{h}{\Lambda} \frac{J_{1}(\Lambda a)}{J_{1}^{\prime}(\Lambda a)}, \\
Y_{0}^{(n)}(z)=\int_{-\infty}^{\infty} d h e^{-j h z} \frac{h^{2}\left[(-1)^{n} e^{j h w}-e^{-j h w}\right]}{2\left(h^{2}-\frac{n^{2} \pi^{2}}{4 w^{2}}\right) \Lambda} \frac{J_{1}(\Lambda a)}{J_{1}^{\prime}(\Lambda a)} \\
Y_{1}^{(n)}(z)=\int_{-\infty}^{\infty} d h e^{-j h z} \frac{h\left[(-1)^{n} e^{j h w}-e^{-j h w}\right] J_{1}^{\prime}(\Lambda a)}{2 \Lambda\left(h^{2}-\frac{n^{2} \pi^{2}}{4 w^{2}}\right) J_{1}(\Lambda a)} \\
Y_{2}(z)=\frac{1}{2 w} \int_{-w}^{w} d \xi \int_{-\infty}^{\infty} d h e^{-j h(z-\xi)} \frac{J_{1}^{\prime}(\Lambda a)}{\Lambda J_{1}(\Lambda a)},
\end{gathered}
$$

$$
Y_{3}(z)=\frac{1}{2 w} \int_{-w}^{w} d \xi \int_{-\infty}^{\infty} d h e^{-j h(z-\xi)} \frac{h^{2} J_{1}(\Lambda a)}{\Lambda^{3} J_{1}^{\prime}(\Lambda a)}
$$$$
Y_{4}^{(n)}(z)=\int_{-\infty}^{\infty} d h e^{-j h z} \frac{h^{3}\left[(-1)^{n} e^{j h w}-e^{-j h w}\right] J_{1}(\Lambda a)}{2 \Lambda^{3}\left(h^{2}-\frac{n^{2} \pi^{2}}{4 w^{2}}\right) J_{1}^{\prime}(\Lambda a)},
$$

$$
Y_{4}^{\prime(n)}(z)=\int_{-\infty}^{\infty} d h e^{-j h z} \frac{h\left[(-1)^{n} e^{j h w}-e^{-j h w}\right]}{2\left(h^{2}-\frac{n^{2} \pi^{2}}{4 w^{2}}\right)} \frac{J_{1}(\Lambda a)}{\Lambda J_{1}^{\prime}(\Lambda a)} .
$$

Here, a new bracket,

$$
\langle\langle\cdots\rangle\rangle_{m}^{s} \equiv \int_{-w}^{w} d z \sin \frac{m \pi(z+w)}{2 w} \cdots
$$

is introduced. The concrete expressions of $\left\langle\left\langle Y_{-2}^{(n)}\right\rangle\right\rangle_{m}^{s}$, $\left\langle\left\langle Y_{-1}\right\rangle\right\rangle_{m}^{s}, \quad\left\langle\left\langle Y_{0}^{(n)}\right\rangle\right\rangle_{m}^{s}, \quad\left\langle\left\langle Y_{0}^{(n)}\right\rangle\right\rangle_{m}^{s}, \quad\left\langle\left\langle Y_{0}^{(n)}\right\rangle\right\rangle_{m}^{s}, \quad\left\langle Y_{1}^{(n)}-Y_{4}^{(n)} /\right.$ $\left.k^{2} \beta^{2} a^{2}\right\rangle, \quad\left\langle\left\langle Y_{1}^{(n)}-Y_{4}^{(n)} / k^{2} \beta^{2} a^{2}\right\rangle\right\rangle_{m}, \quad\left\langle Y_{4}^{\prime(n)}\right\rangle, \quad\left\langle\left\langle Y_{4}^{\prime(n)}\right\rangle\right\rangle_{m}$, $\left\langle Y_{2}-Y_{3} / k^{2} \beta^{2} a^{2}\right\rangle, \quad\left\langle\left\langle Y_{2}-Y_{3} / k^{2} \beta^{2} a^{2}\right\rangle\right\rangle_{m}, \quad\left\langle Y_{5}-Y_{6} /\right.$ $\left.k^{2} \beta^{2} a_{2}^{2}\right\rangle, \quad\left\langle\left\langle Y_{5}-Y_{6} / k^{2} \beta^{2} a_{2}^{2}\right\rangle\right\rangle_{m}, \quad\left\langle Y_{7}^{(n)}-Y_{8}^{(n)} / k^{2} \beta^{2} a_{2}^{2}\right\rangle$, $\left\langle\left\langle Y_{7}^{(n)}(z)-Y_{8}^{(n)}(z) / k^{2} \beta^{2} a_{2}^{2}\right\rangle\right\rangle_{m},\left\langle Y_{9}^{(n)}\right\rangle,\left\langle\left\langle Y_{9}^{(n)}\right\rangle\right\rangle_{m},\left\langle\left\langle Y_{10}(z)\right\rangle\right\rangle_{m}^{s}$, $\left\langle\left\langle Y_{11}^{(n)}\right\rangle\right\rangle_{m}^{s}$ and $\left\langle\left\langle Y_{12}^{(n)}\right\rangle\right\rangle_{m}^{s}$ are given in Appendix D. 
Similarly, the fields on the outer surface of the ceramic $\left(\rho=a_{2}, \theta,-w<z<w\right)$, which are calculated by using Eqs. (A33)-(A36), are expanded as

$$
\begin{gathered}
E_{z}\left(a_{2}\right)=\tilde{E}_{z}\left(a_{2}\right) \cos \theta, \\
H_{\theta}\left(a_{2}\right)=\tilde{H}_{\theta}\left(a_{2}\right) \cos \theta,
\end{gathered}
$$

$$
\begin{gathered}
H_{z}\left(a_{2}\right)=\tilde{H}_{z}\left(a_{2}\right) \sin \theta, \\
E_{\theta}\left(a_{2}\right)=\tilde{E}_{\theta}\left(a_{2}\right) \sin \theta, \\
\tilde{E}_{z}\left(a_{2}\right)=i_{1} \frac{\pi \mathcal{V}_{2}}{w}+i_{1} \sum_{m=1}^{\infty} \frac{\cos \frac{m \pi(z+w)}{2 w} \pi \mathcal{V}_{2}^{(m)},}{w}
\end{gathered}
$$

$$
\begin{aligned}
\tilde{H}_{\theta}\left(a_{2}\right)= & -\frac{j k \beta}{Z_{0}}\left\langle Y_{5}(z)-\frac{Y_{6}(z)}{k^{2} \beta^{2} a_{2}^{2}}\right\rangle i_{1} \mathcal{V}_{2}-\sum_{n=1}^{\infty} \frac{k \beta}{w Z_{0}}\left\langle Y_{7}^{(n)}(z)-\frac{Y_{8}^{(n)}(z)}{k^{2} \beta^{2} a_{2}^{2}}\right\rangle i_{1} \mathcal{V}_{2}^{(n)}-\sum_{n=1}^{\infty} \frac{n \pi}{2 k \beta w^{2} Z_{0} a_{2}}\left\langle Y_{9}^{(n)}\right\rangle i_{1} \mathcal{V}_{1}^{(n)} \\
& +\sum_{m=1}^{\infty} \frac{\cos \frac{m \pi(z+w)}{2 w}}{w}\left[-\sum_{n=1}^{\infty} \frac{k \beta}{w Z_{0}}\left\langle\left\langle Y_{7}^{(n)}(z)-\frac{Y_{8}^{(n)}(z)}{k^{2} \beta^{2} a_{2}^{2}}\right\rangle\right\rangle_{m} i_{1} \mathcal{V}_{2}^{(n)}\right. \\
& \left.-\sum_{n=1}^{\infty} \frac{n \pi}{2 k \beta w^{2} Z_{0} a_{2}}\left\langle\left\langle Y_{9}^{(n)}\right\rangle\right\rangle_{m} i_{1} \mathcal{V}_{1}^{(n)}-\frac{j k \beta}{Z_{0}}\left\langle\left\langle Y_{5}(z)-\frac{Y_{6}(z)}{k^{2} \beta^{2} a_{2}^{2}}\right\rangle\right\rangle_{m} i_{1} \mathcal{V}_{2}\right], \\
& \tilde{H}_{z}\left(a_{2}\right)=\sum_{m=1}^{\infty} \frac{\sin \frac{m \pi(z+w)}{2 w}}{w}\left[-\frac{\left.\left\langle Y_{10}(z)\right\rangle\right\rangle_{m}^{s}}{k \beta Z_{0} a_{2}} i_{1} \mathcal{V}_{2}+\sum_{n=1}^{\infty} \frac{j\left\langle\left\langle Y_{11}^{(n)}(z)\right\rangle\right\rangle_{m}^{s}}{k \beta Z_{0} a_{2} w} i_{1} \mathcal{V}_{2}^{(n)}-\sum_{n=1}^{\infty} \frac{j n \pi\left\langle\left\langle Y_{12}^{(n)}(z)\right\rangle\right\rangle_{m}^{s}}{2 k \beta w^{2} Z_{0}} i_{1} \mathcal{V}_{1}^{(n)}\right], \\
& \tilde{E}_{\theta}\left(a_{2}\right)=\sum_{m=1}^{\infty} \frac{\sin \frac{m \pi(z+w)}{2 w}}{w} \pi i_{1} \mathcal{V}_{1}^{(m)}, \quad(\mathrm{A} 57) \quad Y_{10}(z)=\int_{-\infty}^{\infty} d h e^{-j h z} \frac{\sin (h w)}{h w} \frac{h H_{1}^{(2)}\left(\Lambda a_{2}\right)}{\Lambda H_{1}^{(2)}\left(\Lambda a_{2}\right)},
\end{aligned}
$$

where

$$
\begin{aligned}
& Y_{5}(z)=\frac{1}{2 w} \int_{-w}^{w} d \xi \int_{-\infty}^{\infty} d h e^{-j h(z-\xi)} \frac{H_{1}^{\prime(2)}\left(\Lambda a_{2}\right)}{\Lambda H_{1}^{(2)}\left(\Lambda a_{2}\right)}, \quad(\mathrm{A} 58) \quad Y_{11}^{(n)}(z)=\int_{-\infty}^{\infty} d h e^{-j h z} \frac{h^{2}\left[(-1)^{n} e^{j h w}-e^{-j h w}\right] H_{1}^{(2)}\left(\Lambda a_{2}\right)}{2\left(h^{2}-\frac{n^{2} \pi^{2}}{4 w^{2}} \Lambda H_{1}^{\prime(2)}\left(\Lambda a_{2}\right)\right.}, \\
& Y_{6}(z)=\frac{1}{2 w} \int_{-w}^{w} d \xi \int_{-\infty}^{\infty} d h e^{-j h(z-\xi)} \frac{h^{2} H_{1}^{(2)}\left(\Lambda a_{2}\right)}{\Lambda^{3} H_{1}^{\prime(2)}\left(\Lambda a_{2}\right)}, \quad(\mathrm{A} 59) \\
& Y_{7}^{(n)}(z)=\int_{-\infty}^{\infty} d h e^{-j h z} \frac{h\left[(-1)^{n} e^{j h w}-e^{-j h w}\right] H_{1}^{\prime(2)}\left(\Lambda a_{2}\right)}{2\left(h^{2}-\frac{n^{2} \pi^{2}}{4 w^{2}}\right) \Lambda H_{1}^{(2)}\left(\Lambda a_{2}\right)}, \quad Y_{12}^{(n)}(z)=\int_{-\infty}^{\infty} d h e^{-j h z} \frac{\Lambda\left[(-1)^{n} e^{j h w}-e^{-j h w}\right] H_{1}^{(2)}\left(\Lambda a_{2}\right)}{2\left(h^{2}-\frac{n^{2} \pi^{2}}{4 w^{2}}\right) H_{1}^{\prime(2)}\left(\Lambda a_{2}\right)}
\end{aligned}
$$

$Y_{8}^{(n)}(z)=\int_{-\infty}^{\infty} d h e^{-j h z} \frac{h^{3}\left[(-1)^{n} e^{j h w}-e^{-j h w}\right] H_{1}^{(2)}\left(\Lambda a_{2}\right)}{2\left(h^{2}-\frac{n^{2} \pi^{2}}{4 w^{2}}\right) \Lambda^{3} H_{1}^{\prime(2)}\left(\Lambda a_{2}\right)}$,

$$
Y_{9}^{(n)}(z)=\int_{-\infty}^{\infty} d h e^{-j h z} \frac{h\left[(-1)^{n} e^{j h w}-e^{-j h w}\right] H_{1}^{(2)}\left(\Lambda a_{2}\right)}{2 \Lambda\left(h^{2}-\frac{n^{2} \pi^{2}}{4 w^{2}}\right) H_{1}^{\prime(2)}\left(\Lambda a_{2}\right)},
$$

The transfer coefficients for dipole mode $A_{1}^{(0)}, C_{1}^{(0)}, I_{1}^{(0)}$, $K_{1}^{(0)}, A_{1}^{(m)}, B_{1}^{(m)}, C_{1}^{(m)}, D_{1}^{(m)}, E_{1}^{(m)}, F_{1}^{(m)}, G_{1}^{(m)}, H_{1}^{(m)}, I_{1}^{(m)}$, $J_{1}^{(m)}, K_{1}^{(m)}, L_{1}^{(m)}, M_{1}^{(m)}, N_{1}^{(m)}, O_{1}^{(m)}$ and $P_{1}^{(m)}$ with positive integer $m$ are explicitly expressed in Appendix C 2. Using the coefficients, the fields on $\rho=a$ are transferred to those on $\rho=a_{2}$. The connection conditions between the fields $E_{z}, H_{z}, E_{\theta}, H_{\theta}$ on $\rho=a_{2}$ enable us to obtain the following equations: 


$$
\begin{aligned}
& A_{1}^{(0)} \frac{\pi \tilde{\mathcal{V}}_{2}}{w}+C_{1}^{(0)} \frac{c \beta I_{1}\left(\bar{k} r_{b}\right)}{r_{b} \pi a I_{1}(\bar{k} a)} \frac{\sin k w}{k w}-C_{1}^{(0)} \frac{j k \beta}{Z_{0}}\left\langle Y_{2}(z)-\frac{Y_{3}(z)}{k^{2} \beta^{2} a^{2}}\right\rangle \tilde{\mathcal{V}}_{2} \\
& -C_{1}^{(0)} \sum_{n=1}^{\infty} \tilde{\mathcal{V}}_{1}^{(n)} \frac{n \pi}{2 w^{2} Z_{0} k \beta a}\left\langle Y_{4}^{(n)}(z)\right\rangle-C_{1}^{(0)} \sum_{n=1}^{\infty} \tilde{\mathcal{V}}_{2}^{(n)} \frac{k \beta}{w Z_{0}}\left\langle Y_{1}^{(n)}(z)-\frac{Y_{4}^{(n)}(z)}{k^{2} \beta^{2} a^{2}}\right\rangle=\frac{\pi \mathcal{V}_{2}}{w}, \\
& 0=-\frac{j k \beta}{Z_{0}}\left\langle Y_{5}(z)-\frac{Y_{6}(z)}{k^{2} \beta^{2} a_{2}^{2}}\right\rangle \mathcal{V}_{2}-\sum_{n=1}^{\infty} \frac{k \beta}{w Z_{0}}\left\langle Y_{7}^{(n)}(z)-\frac{Y_{8}^{(n)}(z)}{k^{2} \beta^{2} a_{2}^{2}}\right\rangle \mathcal{V}_{2}^{(n)}-\sum_{n=1}^{\infty} \frac{n \pi}{2 k \beta w^{2} Z_{0} a_{2}}\left\langle Y_{9}^{(n)}\right\rangle \mathcal{V}_{1}^{(n)} \\
& \left.-I_{1}^{(0)} \frac{\pi}{w} \tilde{\mathcal{V}}_{2}-K_{1}^{(0)} \frac{c \beta I_{1}\left(\bar{k} r_{b}\right)}{r_{b} \pi a I_{1}(\bar{k} a)} \frac{\sin k w}{k w}+K_{1}^{(0)} \frac{j k \beta}{Z_{0}}\left\langle Y_{2}(z)-\frac{Y_{3}(z)}{k^{2} \beta^{2} a^{2}}\right)\right\rangle \tilde{\mathcal{V}}_{2} \\
& +K_{1}^{(0)} \sum_{n=1}^{\infty} \tilde{\mathcal{V}}_{1}^{(n)} \frac{n \pi}{2 w^{2} Z_{0} k \beta a}\left\langle Y_{4}^{(n)}(z)\right\rangle+K_{1}^{(0)} \sum_{n=1}^{\infty} \tilde{\mathcal{V}}_{2}^{(n)} \frac{k \beta}{w Z_{0}}\left\langle Y_{1}^{(n)}(z)-\frac{Y_{4}^{(n)}(z)}{k^{2} \beta^{2} a^{2}}\right\rangle \\
& A_{1}^{(m)} \pi \tilde{\mathcal{V}}_{2}^{(m)}-\sum_{n=1}^{\infty} \frac{B_{1}^{(m)}}{j w a k \beta Z_{0}}\left\langle\left\langle Y_{0}^{(n)}(z)\right\rangle\right\rangle_{m}^{s} \tilde{\mathcal{V}}_{2}^{(n)}-\sum_{n=1}^{\infty} C_{1}^{(m)} \frac{k \beta}{w Z_{0}}\left\langle\left\langle Y_{1}^{(n)}(z)-\frac{Y_{4}^{(n)}(z)}{k^{2} \beta^{2} a^{2}}\right\rangle\right\rangle_{m} \tilde{\mathcal{V}}_{2}^{(n)} \\
& +\sum_{n=1}^{\infty} B_{1}^{(m)} \frac{n \pi}{2 j k \beta Z_{0} w^{2}}\left\langle\left\langle Y_{-2}^{(n)}\right\rangle\right\rangle_{m}^{s} \tilde{\mathcal{V}}_{1}^{(n)}-\sum_{n=1}^{\infty} C_{1}^{(m)} \frac{n \pi}{2 w^{2} k \beta a Z_{0}}\left\langle\left\langle Y_{4}^{(n)}\right\rangle\right\rangle_{m} \tilde{\mathcal{V}}_{1}^{(n)}-\pi \mathcal{V}_{2}^{(m)}+D_{1}^{(m)} \pi \tilde{\mathcal{V}}_{2}^{(m)} \\
& =-C_{1}^{(m)} \frac{j c \beta k I_{1}\left(\bar{k} r_{b}\right)\left[e^{j k w}-(-1)^{m} e^{-j k w}\right]}{r_{b} \pi a I_{1}(\bar{k} a)\left(\frac{m^{2} \pi^{2}}{4 w^{2}}-k^{2}\right)}+\frac{\tilde{\mathcal{V}}_{2}}{a k \beta Z_{0}} B_{1}^{(m)}\left\langle\left\langle Y_{-1}(z)\right\rangle_{m}^{s}+C_{1}^{(m)} \frac{j k \beta}{Z_{0}}\left\langle\left\langle Y_{2}(z)-\frac{Y_{3}(z)}{k^{2} \beta^{2} a^{2}}\right\rangle\right\rangle_{m} \tilde{\mathcal{V}}_{2}\right. \\
& I_{1}^{(m)} \pi \tilde{\mathcal{V}}_{2}^{(m)}-\sum_{n=1}^{\infty} \frac{J_{1}^{(m)}}{j w a k \beta Z_{0}}\left\langle\left\langle Y_{0}^{(n)}(z)\right\rangle_{m}^{s} \tilde{\mathcal{V}}_{2}^{(n)}-\sum_{n=1}^{\infty} K_{1}^{(m)} \frac{k \beta}{w Z_{0}}\left\langle\left\langle Y_{1}^{(n)}(z)-\frac{Y_{4}^{(n)}(z)}{k^{2} \beta^{2} a^{2}}\right\rangle\right\rangle_{m} \tilde{\mathcal{V}}_{2}^{(n)}+\sum_{n=1}^{\infty} J_{1}^{(m)} \frac{n \pi}{2 j k \beta Z_{0} w^{2}}\left\langle\left\langle Y_{-2}^{(n)}\right\rangle\right\rangle_{m}^{s} \tilde{\mathcal{V}}_{1}^{(n)}\right. \\
& -\sum_{n=1}^{\infty} K_{1}^{(m)} \frac{n \pi}{2 w^{2} k \beta a Z_{0}}\left\langle\left\langle Y_{4}^{(n)}\right\rangle\right\rangle_{m} \tilde{\mathcal{V}}_{1}^{(n)}+L_{1}^{(m)} \pi \tilde{\mathcal{V}}_{1}^{(m)}+\sum_{n=1}^{\infty} \frac{k \beta}{w Z_{0}}\left\langle\left\langle Y_{7}^{(n)}(z)-\frac{Y_{8}^{(n)}(z)}{k^{2} \beta^{2} a_{2}^{2}}\right\rangle\right\rangle_{m} \mathcal{V}_{2}^{(n)}+\frac{m \pi}{2 k \beta w^{2} Z_{0} a_{2}}\left\langle\left\langle Y_{9}^{(n)}\right\rangle\right\rangle_{m} \mathcal{V}_{1}^{(n)} \\
& =\frac{\tilde{\mathcal{V}}_{2}}{a k \beta Z_{0}} J_{1}^{(m)}\left\langle\left\langle Y_{-1}(z)\right\rangle\right\rangle_{m}^{s}-K_{1}^{(m)} \frac{j c \beta k I_{1}\left(\bar{k} r_{b}\right)\left[e^{j k w}-(-1)^{m} e^{-j k w}\right]}{r_{b} \pi a I_{1}(\bar{k} a)\left(\frac{m^{2} \pi^{2}}{4 w^{2}}-k^{2}\right)}+K_{1}^{(m)} \frac{j k \beta}{Z_{0}}\left\langle\left\langle Y_{2}(z)-\frac{Y_{3}(z)}{k^{2} \beta^{2} a^{2}}\right\rangle\right\rangle_{m} \tilde{\mathcal{V}}_{2} \\
& -\frac{j k \beta}{Z_{0}}\left\langle\left\langle Y_{5}(z)-\frac{Y_{6}(z)}{k^{2} \beta^{2} a_{2}^{2}}\right\rangle\right\rangle_{m} \mathcal{V}_{2} \\
& E_{1}^{(m)} \pi \tilde{\mathcal{V}}_{2}^{(m)}-\sum_{n=1}^{\infty} \frac{F_{1}^{(m)}}{j w a k \beta Z_{0}}\left\langle\left\langle Y_{0}^{(n)}(z)\right\rangle\right\rangle_{m}^{s} \tilde{\mathcal{V}}_{2}^{(n)}+\sum_{n=1}^{\infty} F_{1}^{(m)} \frac{n \pi}{2 j k \beta Z_{0} w^{2}}\left\langle\left\langle Y_{-2}^{(n)}\right\rangle\right\rangle_{m}^{s} \tilde{\mathcal{V}}_{1}^{(n)}+H_{1}^{(m)} \pi \tilde{\mathcal{V}}_{1}^{(m)} \\
& +\sum_{n=1}^{\infty} \frac{\left\langle\left\langle Y_{11}^{(n)}(z)\right\rangle\right\rangle_{m}^{s}}{j k \beta Z_{0} a_{2} w} \mathcal{V}_{2}^{(n)}-\sum_{n=1}^{\infty} \frac{n \pi\left\langle\left\langle Y_{12}^{(n)}(z)\right\rangle\right\rangle_{m}^{s}}{j 2 k \beta w^{2} Z_{0}} \mathcal{V}_{1}^{(n)}+G_{1}^{(m)}\left[\frac{j c \beta k I_{1}\left(\bar{k} r_{b}\right)\left[e^{j k w}-(-1)^{m} e^{-j k w}\right]}{r_{b} \pi a I_{1}(\bar{k} a)\left(\frac{m^{2} \pi^{2}}{4 w^{2}}-k^{2}\right)}\right. \\
& -\frac{j k \beta}{Z_{0}}\left\langle\left\langle Y_{2}(z)-\frac{Y_{3}(z)}{k^{2} \beta^{2} a^{2}}\right\rangle\right\rangle_{m} \tilde{\mathcal{V}}_{2}-\sum_{n=1}^{\infty} \frac{k \beta}{w Z_{0}}\left\langle\left\langle Y_{1}^{(n)}(z)-\frac{Y_{4}^{(n)}(z)}{k^{2} \beta^{2} a^{2}}\right\rangle\right\rangle_{m} \tilde{\mathcal{V}}_{2}^{(n)}-\sum_{n=1}^{\infty} \frac{n \pi \tilde{\mathcal{V}}_{1}^{(n)}}{2 w^{2} k \beta a Z_{0}}\left\langle\left\langle Y_{4}^{\prime(n)}\right\rangle_{m}\right] \\
& =\frac{\tilde{\mathcal{V}}_{2}}{a k \beta Z_{0}} F_{1}^{(m)}\left\langle\left\langle Y_{-1}(z)\right\rangle\right\rangle_{m}^{s}-\frac{\left\langle\left\langle Y_{10}(z)\right\rangle\right\rangle_{m}^{s}}{k \beta Z_{0} a_{2}} \mathcal{V}_{2}
\end{aligned}
$$




$$
\begin{aligned}
& M_{1}^{(m)} \pi \tilde{\mathcal{V}}_{2}^{(m)}-\sum_{n=1}^{\infty} \frac{N_{1}^{(m)}}{j w a k \beta Z_{0}}\left\langle\left\langle Y_{0}^{(n)}(z)\right\rangle\right\rangle_{m}^{s} \tilde{\mathcal{V}}_{2}^{(n)}-\sum_{n=1}^{\infty} O_{1}^{(m)} \frac{k \beta}{w Z_{0}}\left\langle\left\langle Y_{1}^{(n)}(z)-\frac{Y_{4}^{(n)}(z)}{k^{2} \beta^{2} a^{2}}\right\rangle\right\rangle_{m} \tilde{\mathcal{V}}_{2}^{(n)} \\
& \quad+\sum_{n=1}^{\infty} N_{1}^{(m)} \frac{n \pi}{2 j k \beta Z_{0} w^{2}}\left\langle\left\langle Y_{-2}^{(n)}\right\rangle\right\rangle_{m}^{s} \tilde{\mathcal{V}}_{1}^{(n)}-\sum_{n=1}^{\infty} O_{1}^{(m)} \frac{n \pi}{2 w^{2} k \beta a Z_{0}}\left\langle\left\langle Y_{4}^{(n)}\right\rangle_{m} \tilde{\mathcal{V}}_{1}^{(n)}+P_{1}^{(m)} \pi \tilde{\mathcal{V}}_{1}^{(m)}-\pi \mathcal{V}_{1}^{(m)}\right. \\
& =\frac{\tilde{\mathcal{V}}_{2}}{a k \beta Z_{0}} N_{1}^{(m)}\left\langle\left\langle Y_{-1}(z)\right\rangle\right\rangle_{m}^{s}-O_{1}^{(m)} \frac{j c \beta k I_{1}\left(\bar{k} r_{b}\right)\left[e^{j k w}-(-1)^{m} e^{-j k w}\right]}{r_{b} \pi a I_{1}(\bar{k} a)\left(\frac{m^{2} \pi^{2}}{4 w^{2}}-k^{2}\right)}+O_{1}^{(m)} \frac{j k \beta}{Z_{0}}\left\langle\left\langle Y_{2}(z)-\frac{Y_{3}(z)}{k^{2} \beta^{2} a^{2}}\right\rangle\right\rangle_{m} \tilde{\mathcal{V}}_{2} .
\end{aligned}
$$

The expansion coefficients $\mathcal{V}_{1}^{(m)}, \mathcal{V}_{2}, \mathcal{V}_{2}^{(m)}, \tilde{\mathcal{V}}_{1}^{(m)}, \tilde{\mathcal{V}}_{2}$ and $\tilde{\mathcal{V}}_{2}^{(m)}$ are obtained by solving Eqs. (A66)-(A71) for any order of $m$

By substituting the solutions into Eq. (12) in the text and using the Panofsky-Wenzel theorem [5,14], we finally obtain the expression for the transverse impedance as in Eq. (17) in the text.

\section{APPENDIX B: CONCRETE EXPRESSIONS OF $\langle\boldsymbol{\alpha}\rangle,\langle\boldsymbol{J}\rangle,\left\langle\boldsymbol{J}^{(m)}\right\rangle,\left\langle\langle\boldsymbol{\alpha}\rangle_{m},\langle\langle\boldsymbol{J}\rangle\rangle_{m},\left\langle\left\langle\boldsymbol{J}^{(n)}\right\rangle\right\rangle_{m},\langle\boldsymbol{Y}\rangle_{m},\left\langle\boldsymbol{Y}^{(m)}\right\rangle\right.$, $\langle\langle\boldsymbol{Y}\rangle\rangle_{m}$ AND $\left\langle\left\langle\boldsymbol{Y}^{(n)}\right\rangle_{m}\right.$}

The brackets $\langle\cdots\rangle$ and $\langle\langle\cdots\rangle\rangle_{m}$ are previously defined as in Eqs. (A18) and (A19) in Appendix A 1, respectively. The functions $\alpha(z), J(z), J^{(m)}(z), Y(z), Y^{(m)}(z)$ are given by Eqs. (A13)-(A17) in Appendix A 1, respectively. Hence, by substituting the functions into Eqs. (A18) and (A19), we obtain the formal expressions of $\langle\alpha\rangle,\langle J\rangle,\left\langle J^{(m)}\right\rangle,\langle\langle\alpha\rangle\rangle_{m}$, $\langle\langle J\rangle\rangle_{m},\left\langle\left\langle J^{(n)}\right\rangle_{m},\langle Y\rangle_{m},\left\langle Y^{(m)}\right\rangle,\langle\langle Y\rangle\rangle_{m}\right.$ and $\left\langle\left\langle Y^{(n)}\right\rangle\right\rangle_{m}$. After these expressions are integrated over $z, h$-integrations remain in all cases. The $h$-integration can be performed by using the expansion formulas $[10,23]$ for Bessel functions,

$$
\begin{gathered}
\frac{J_{0}^{\prime}(z)}{z J_{0}(z)}=\sum_{s=1}^{\infty} \frac{2}{z^{2}-j_{0, s}^{2}}, \\
\frac{1}{\sqrt{z}} \frac{H_{1}^{(2)}(\sqrt{z})}{H_{0}^{(2)}(\sqrt{z})}=\int_{0}^{\infty} d \zeta \frac{2}{\pi^{2} \zeta(\zeta+z) H_{0}^{(1)}\left(e^{\frac{\pi}{2} j} \sqrt{\zeta}\right) H_{0}^{(2)}\left(e^{\frac{\pi}{2} j} \sqrt{\zeta}\right)},
\end{gathered}
$$

where $j_{0, s}, H_{m}^{(1)}(z)$ and $H_{m}^{(2)}(z)$ are the $s$ th zeros of $J_{0}(z)$, the Hankel function of the first and the second kind, respectively. The integration of the Bessel functions can be carried out by simply picking up residues in the complex plane $h$. The path of integration is chosen to be below the poles for $h<0$ and above the poles for $h>0$.

Finally, we obtain the following expressions:

$$
\begin{aligned}
& \langle\alpha\rangle=\frac{\beta c I_{1}(\bar{k} \sigma)}{\pi \sigma \bar{k} a I_{0}(\bar{k} a)} \frac{\sin k w}{k w}, \\
& \langle\langle\alpha\rangle\rangle_{m}=-\frac{\beta c I_{1}(\bar{k} \sigma)}{\pi \sigma \bar{k} a I_{0}(\bar{k} a)} \frac{k\left[(-1)^{m} e^{-j k w}-e^{j k w}\right]}{j\left(k^{2}-\frac{m^{2} \pi^{2}}{4 w^{2}}\right)}, \\
& \langle J(z)\rangle=-\sum_{s=1}^{\infty} \frac{2 \pi a}{w b_{s}^{2}}-\sum_{s=1}^{\infty} \frac{\pi a^{2}\left(e^{-j 2 \frac{b_{s}}{a} w}-1\right)}{j w^{2} b_{s}^{3}},
\end{aligned}
$$

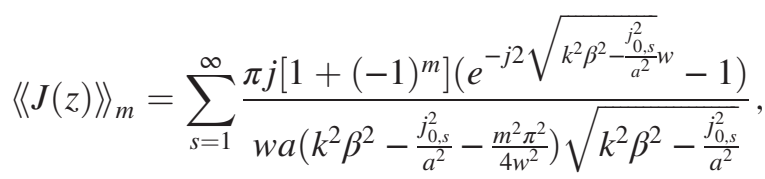

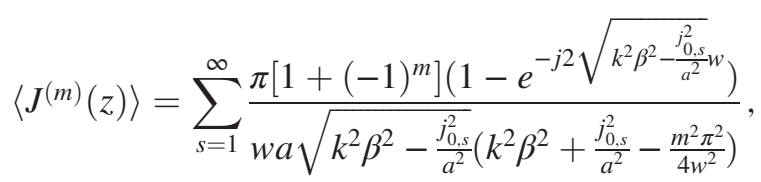

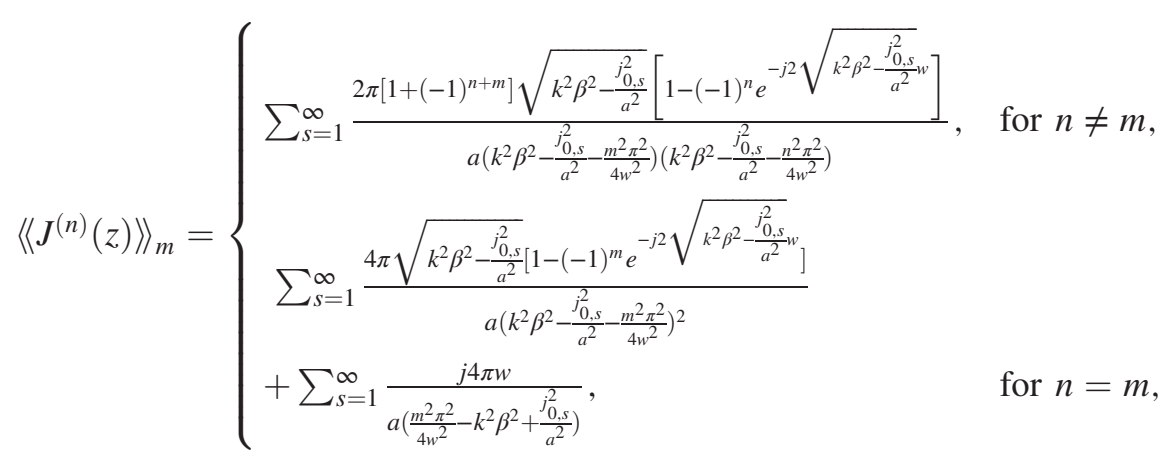




$$
\begin{aligned}
& \langle Y(z)\rangle=-\int_{0}^{\infty} d \zeta \frac{2}{w \pi a_{2} \zeta\left(k^{2} \beta^{2}+\frac{\zeta}{a_{2}^{2}}\right) H_{0}^{(1)}\left(e^{j \frac{\pi}{2}} \sqrt{\zeta}\right) H_{0}^{(2)}\left(e^{j \frac{\pi}{2}} \sqrt{\zeta}\right)} \\
& -\int_{0}^{\infty} d \zeta \frac{\left(e^{-j 2 w} \sqrt{k^{2} \beta^{2}+\frac{\zeta}{a_{2}^{2}}}-1\right)}{j w^{2} \pi a_{2} \zeta\left(k^{2} \beta^{2}+\frac{\zeta}{a_{2}^{2}}\right)^{\frac{3}{2}} H_{0}^{(1)}\left(e^{j \frac{\pi}{2}} \sqrt{\zeta}\right) H_{0}^{(2)}\left(e^{j \frac{\pi}{2}} \sqrt{\zeta}\right)}, \\
& \langle\langle Y(z)\rangle\rangle_{m}=-\int_{0}^{\infty} d \zeta \frac{j\left[1+(-1)^{m}\right]\left(1-e^{-j 2 \sqrt{\frac{\zeta}{a_{2}^{2}+k^{2} \beta^{2}} w}}\right)}{w \pi a_{2} \zeta \sqrt{\frac{\zeta}{a_{2}^{2}}+k^{2} \beta^{2}}\left(\frac{\zeta}{a_{2}^{2}}+k^{2} \beta^{2}-\frac{m^{2} \pi^{2}}{4 w^{2}}\right) H_{0}^{(1)}\left(e^{\frac{\pi}{2} j} \sqrt{\zeta}\right) H_{0}^{(2)}\left(e^{\frac{\pi}{2} j} \sqrt{\zeta}\right)}, \\
& \left\langle Y^{(m)}(z)\right\rangle=\int_{0}^{\infty} d \zeta \frac{\left[1+(-1)^{m}\right]\left(1-e^{-j 2 \sqrt{\frac{\zeta}{a_{2}^{2}+k^{2} \beta^{2}} w}}\right)}{w \pi a_{2} \zeta\left(\frac{\zeta}{a_{2}^{2}}+k^{2} \beta^{2}-\frac{m^{2} \pi^{2}}{4 w^{2}}\right) \sqrt{\frac{\zeta}{a_{2}^{2}}+k^{2} \beta^{2}} H_{0}^{(1)}\left(e^{\frac{\pi}{2} j} \sqrt{\zeta}\right) H_{0}^{(2)}\left(e^{\frac{\pi}{2} j} \sqrt{\zeta}\right)}, \\
& \left\langle Y^{(n)}(z)\right\rangle_{m}= \begin{cases}\int_{0}^{\infty} d \zeta \frac{2\left[1+(-1)^{m+n}\right] \sqrt{\frac{\zeta}{a_{2}^{2}}+k^{2} \beta^{2}}\left[1-(-1)^{n} e^{\left.-j 2 \sqrt{\frac{\zeta}{a_{2}^{2}+k^{2} \beta^{2}} w}\right]}\right.}{\pi a_{2} \zeta\left(\frac{\zeta}{a_{2}^{2}}+k^{2} \beta^{2}-\frac{m^{2} \pi^{2}}{4 w^{2}}\right)\left(\frac{\zeta}{a_{2}^{2}}+k^{2} \beta^{2}-\frac{n^{2} \pi^{2}}{4 w^{2}}\right) H_{0}^{(1)}\left(e^{\frac{\pi}{2} j} \sqrt{\zeta}\right) H_{0}^{(2)}\left(e^{\frac{\pi}{2} j} \sqrt{\zeta}\right)}, & \text { for } n \neq m, \\
\int_{0}^{\infty} d \zeta \frac{4 \sqrt{\frac{\zeta}{a_{2}^{2}}+k^{2} \beta^{2}}\left[1-(-1)^{m} e^{-j 2} \sqrt{\frac{\zeta}{a_{2}^{2}+k^{2} \beta^{2} w}}\right]}{\pi a_{2} \zeta\left(\frac{\zeta}{a_{2}^{2}}+k^{2} \beta^{2}-\frac{m^{2} \pi^{2}}{4 w^{2}}\right)^{2} H_{0}^{(1)}\left(e^{\frac{\pi}{2} j} \sqrt{\zeta}\right) H_{0}^{(2)}\left(e^{\frac{\pi}{2} j} \sqrt{\zeta}\right)} & \\
+\int_{0}^{\infty} d \zeta \frac{j w}{\pi a_{2} \zeta\left(\frac{m^{2} \pi^{2}}{4 w^{2}}-\frac{\zeta}{a_{2}^{2}}-k^{2} \beta^{2}\right) H_{0}^{(1)}\left(e^{\frac{\pi}{2} j} \sqrt{\zeta}\right) H_{0}^{(2)}\left(e^{\frac{\pi}{2} j} \sqrt{\zeta}\right)}, & \text { for } n=m,\end{cases}
\end{aligned}
$$

where $m, n$ are positive integers, $b_{s}^{2}=k^{2} \beta^{2} a^{2}-j_{0, s}^{2}=$ $-\beta_{s}^{2}$, and $b_{s}$ approaches $-j \beta_{s}$ for $j_{0, s}>k \beta a$. Though it looks like $\zeta=m^{2} \pi^{2} a_{2}^{2} / 4 w^{2}-k^{2} \beta^{2} a_{2}^{2}$ is a singular point in some integrands, it disappears after simplifying the relevant terms.

\section{APPENDIX C: TRANSFER COEFFICIENTS THAT TRANSFORM THE FIELDS ON $\rho=a$ TO THOSE ON $\rho=a_{2}$}

In this Appendix, let us derive the coefficients that transfer the fields on the inner surface $(\rho=a)$ to those on the outer surface $\left(\rho=a_{2}\right)$ of the ceramic break. First, let us consider the ceramic break made of a single material with conductivity $\sigma_{c}$, relative dielectric constant $\epsilon^{\prime}$ and relative permeability $\mu^{\prime}$. Next, we derive the expressions for the ceramic break made of a ceramic with relative dielectric constant $\epsilon^{\prime}$ whose inner surface is coated with thin TiN with conductivity $\sigma_{\mathrm{TiN}}$.

\section{Monopole mode}

Because the ceramic (with conductivity $\sigma_{c}$, relative dielectric constant $\epsilon^{\prime}$ and relative permeability $\mu^{\prime}$ ) break is sandwiched by the perfectly conductive chambers on the both sides, the fields for monopole mode in the ceramic are expanded by using sinusoidal functions as

$$
\begin{aligned}
& E_{z}=\frac{1}{2 w} \tilde{\tilde{E}}_{z}^{(0)}+\frac{1}{w} \sum_{m=1}^{\infty} \cos \frac{m \pi(z+w)}{2 w} \tilde{\tilde{E}}_{z}^{(m)}, \\
& H_{\theta}=\frac{1}{2 w} \tilde{\tilde{H}}_{\theta}^{(0)}+\frac{1}{w} \sum_{m=1}^{\infty} \cos \frac{m \pi(z+w)}{2 w} \tilde{\tilde{H}}_{\theta}^{(m)} .
\end{aligned}
$$

Substituting them into Maxwell equations

$$
\operatorname{rot} E=-\frac{\partial B}{\partial t}=-\mu^{\prime} \mu_{0} \frac{\partial H}{\partial t}=-j k \beta \mu^{\prime} Z_{0} H
$$

$r o t H=\sigma_{c} E+\frac{\partial D}{\partial t}=\left(\frac{\sigma_{c} Z_{0}}{j k \beta}+\epsilon^{\prime}\right) \frac{j k \beta}{Z_{0}} E \equiv \tilde{\epsilon} j \omega \epsilon_{0} E$,

the solutions are given as

$$
\tilde{\tilde{E}}_{z}^{(m)}=\tilde{\Gamma}_{0} I_{0}\left(\mu_{m} \rho\right)+\Gamma_{0} K_{0}\left(\mu_{m} \rho\right),
$$




$$
\tilde{\tilde{H}}_{\theta}^{(m)}=\frac{j k \beta \tilde{\varepsilon}}{Z_{0} \mu_{m}}\left[\tilde{\Gamma}_{0} I_{0}^{\prime}\left(\mu_{m} \rho\right)+\Gamma_{0} K_{0}^{\prime}\left(\mu_{m} \rho\right)\right]
$$

where $I_{n}(z)$ and $K_{n}(z)$ are the modified Bessel functions, $\tilde{\Gamma}_{0}$ and $\Gamma_{0}$ are arbitrary coefficients and

$$
\mu_{m}=\sqrt{\frac{m^{2} \pi^{2}}{4 w^{2}}-k^{2} \beta^{2} \tilde{\epsilon} \mu^{\prime}} .
$$

Using the solutions, the fields on $\rho=a$ are transferred to those on $\rho=a_{2}$ as

$$
\left(\begin{array}{l}
\tilde{\tilde{E}}_{z}^{(m)}\left(a_{2}\right) \\
\tilde{\tilde{H}}_{\theta}^{(m)}\left(a_{2}\right)
\end{array}\right)=\mathcal{M}_{0}\left(a_{2}, a\right)\left(\begin{array}{c}
\tilde{\tilde{E}}_{z}^{(m)}(a) \\
\tilde{\tilde{H}}_{\theta}^{(m)}(a)
\end{array}\right),
$$

where

$$
\mathcal{M}_{0}\left(a_{2}, a\right)=\left(\begin{array}{cc}
A_{0}^{(m)} & C_{0}^{(m)} \\
I_{0}^{(m)} & K_{0}^{(m)}
\end{array}\right),
$$

new coefficients $A_{0}^{(m)}, C_{0}^{(m)}, I_{0}^{(m)}$ and $K_{0}^{(m)}$ for monopole mode are introduced, which are given by

$$
A_{0}^{(m)}=\mu_{m} a\left[I_{0}^{\prime}\left(\mu_{m} a\right) K_{0}\left(\mu_{m} a_{2}\right)-I_{0}\left(\mu_{m} a_{2}\right) K_{0}^{\prime}\left(\mu_{m} a\right)\right],
$$

$$
C_{0}^{(m)}=-\frac{j Z_{0} \mu_{m}^{2} a\left[I_{0}\left(\mu_{m} a_{2}\right) K_{0}\left(\mu_{m} a\right)-I_{0}\left(\mu_{m} a\right) K_{0}\left(\mu_{m} a_{2}\right)\right]}{k \beta \tilde{\epsilon}},
$$

$$
I_{0}^{(m)}=\frac{j k \beta a \tilde{\epsilon}\left[I_{0}^{\prime}\left(\mu_{m} a\right) K_{0}^{\prime}\left(\mu_{m} a_{2}\right)-I_{0}^{\prime}\left(\mu_{m} a_{2}\right) K_{0}^{\prime}\left(\mu_{m} a\right)\right]}{Z_{0}},
$$

$$
K_{0}^{(m)}=\mu_{m} a\left[I_{0}{ }^{\prime}\left(\mu_{m} a_{2}\right) K_{0}\left(\mu_{m} a\right)-I_{0}\left(\mu_{m} a\right) K_{0}^{\prime}\left(\mu_{m} a_{2}\right)\right],
$$

especially when $m=0$, they are rewritten as

$$
\begin{gathered}
A_{0}^{(0)}=-\frac{\pi \kappa_{\mathrm{cer}} a\left[Y_{1}\left(\kappa_{\mathrm{cer}} a\right) J_{0}\left(\kappa_{\mathrm{cer}} a_{2}\right)-J_{1}\left(\kappa_{\mathrm{cer}} a\right) Y_{0}\left(\kappa_{\mathrm{cer}} a_{2}\right)\right]}{2}, \\
C_{0}^{(0)}=j \frac{\pi \kappa_{\mathrm{cer}}^{2} a Z_{0}\left[-Y_{0}\left(\kappa_{\mathrm{cer}} a\right) J_{0}\left(\kappa_{\mathrm{cer}} a_{2}\right)+J_{0}\left(\kappa_{\mathrm{cer}} a\right) Y_{0}\left(\kappa_{\mathrm{cer}} a_{2}\right)\right]}{2 \beta \tilde{\epsilon} k}, \\
I_{0}^{(0)}=-\frac{j \beta \tilde{\epsilon} k \pi a\left[Y_{1}\left(\kappa_{\mathrm{cer}} a\right) J_{1}\left(\kappa_{\mathrm{cer}} a_{2}\right)-J_{1}\left(\kappa_{\mathrm{cer}} a\right) Y_{1}\left(\kappa_{\mathrm{cer}} a_{2}\right)\right]}{2 Z_{0}}, \\
K_{0}^{(0)}=-\frac{\pi \kappa_{\mathrm{cer}} a\left[-Y_{0}\left(\kappa_{\mathrm{cer}} a\right) J_{1}\left(\kappa_{\mathrm{cer}} a_{2}\right)+J_{0}\left(\kappa_{\mathrm{cer}} a\right) Y_{1}\left(\kappa_{\mathrm{cer}} a_{2}\right)\right]}{2},
\end{gathered}
$$

by using Bessel functions $J_{n}(z)$ and $Y_{n}(z)$, where

$$
\kappa_{\mathrm{cer}}=\sqrt{k^{2} \beta^{2} \tilde{\epsilon} \mu^{\prime}} .
$$

In general, a ceramic break may be made of different materials. When it has a multilayered structure in the radial direction, the transfer coefficients for the ceramic break are derived by taking the dot product on the different matrices $\mathcal{M}_{0}$ (that correspond to the different materials) along the radial direction.

As a special case, let us consider the case where the ceramic break is made of a ceramic with relative dielectric constant $\epsilon^{\prime}$, whose inner surface is coated with thin TiN with thickness $t$ and conductivity $\sigma_{\mathrm{TiN}}$. Let us approximate the solution in the thin TiN coating by neglecting the higher order mode $(m>0)$. Accordingly, the parameter $\mu_{m}$ in the solution is replaced by Eq. (39). Further, the modified Bessel functions are approximated as [13]

$$
\begin{aligned}
& I_{\nu}\left(\kappa_{\mathrm{TiN}} a\right) \sim \frac{e^{\kappa_{\mathrm{TiN}} a}}{\sqrt{2 \pi \kappa_{\mathrm{TiN}} a}}, \quad I_{\nu}\left[\kappa_{\mathrm{TiN}}(a+t)\right] \sim \frac{e^{\kappa_{\mathrm{TiN}}(a+t)}}{\sqrt{2 \pi \kappa_{\mathrm{TiN}} a}}, \\
& K_{\nu}\left(\kappa_{\mathrm{TiN}} a\right) \sim \sqrt{\frac{\pi}{2 \kappa_{\mathrm{TiN}} a}} e^{-\kappa_{\mathrm{TiN}} a}, \\
& K_{\nu}\left[\kappa_{\mathrm{TiN}}(a+t)\right] \sim \sqrt{\frac{\pi}{2 \kappa_{\mathrm{TiN}} a}} e^{-\kappa_{\mathrm{TiN}}(a+t),}
\end{aligned}
$$

for $\kappa_{\mathrm{TiN}} a \gg 1$ and $\kappa_{\mathrm{TiN}}(a+t) \gg 1$. 
Finally, we obtain the transfer coefficients for the ceramic with the thin TiN coating as

$$
\begin{aligned}
& A_{0}^{(m)} \simeq \mu_{m}^{\epsilon} a\left[I_{0}^{\prime}\left(\mu_{m}^{\epsilon} a\right) K_{0}\left(\mu_{m}^{\epsilon} a_{2}\right)-I_{0}\left(\mu_{m}^{\epsilon} a_{2}\right) K_{0}^{\prime}\left(\mu_{m}^{\epsilon} a\right)\right] \cosh \kappa_{\mathrm{TiN}} t \\
& -\frac{j Z_{0}\left(\mu_{m}^{\epsilon}\right)^{2} a \sigma_{\mathrm{TiN}}\left[I_{0}\left(\mu_{m}^{\epsilon} a_{2}\right) K_{0}\left(\mu_{m}^{\epsilon} a\right)-I_{0}\left(\mu_{m}^{\epsilon} a\right) K_{0}\left(\mu_{m}^{\epsilon} a_{2}\right)\right] \sinh \kappa_{\mathrm{TiN}} t}{k \beta \epsilon^{\prime} \kappa_{\mathrm{TiN}}} \\
& =\frac{\tilde{\mu}_{m} a \pi\left[J_{1}\left(\tilde{\mu}_{m} a\right) Y_{0}\left(\tilde{\mu}_{m} a_{2}\right)-J_{0}\left(\tilde{\mu}_{m} a_{2}\right) Y_{1}\left(\tilde{\mu}_{m} a\right)\right]}{2} \cosh \kappa_{\mathrm{TiN}} t \\
& -\frac{j Z_{0} \tilde{\mu}_{m}^{2} a \sigma_{\mathrm{TiN}} \pi\left[J_{0}\left(\tilde{\mu}_{m} a_{2}\right) Y_{0}\left(\tilde{\mu}_{m} a\right)-J_{0}\left(\tilde{\mu}_{m} a\right) Y_{0}\left(\tilde{\mu}_{m} a_{2}\right)\right]}{2 k \beta \epsilon^{\prime} \kappa_{\mathrm{TiN}}} \sinh \kappa_{\mathrm{TiN}} t, \\
& C_{0}^{(m)} \simeq \frac{\mu_{m}^{\epsilon} a\left[I_{0}^{\prime}\left(\mu_{m}^{\epsilon} a\right) K_{0}\left(\mu_{m}^{\epsilon} a_{2}\right)-I_{0}\left(\mu_{m}^{\epsilon} a_{2}\right) K_{0}^{\prime}\left(\mu_{m}^{\epsilon} a\right)\right] \kappa_{\mathrm{TiN}} \sinh \kappa_{\mathrm{TiN}} t}{\sigma_{\mathrm{TiN}}} \\
& -\frac{j Z_{0}\left(\mu_{m}^{\epsilon}\right)^{2} a\left[I_{0}\left(\mu_{m}^{\epsilon} a_{2}\right) K_{0}\left(\mu_{m}^{\epsilon} a\right)-I_{0}\left(\mu_{m}^{\epsilon} a\right) K_{0}\left(\mu_{m}^{\epsilon} a_{2}\right)\right] \cosh \kappa_{\mathrm{TiN}} t}{k \beta \epsilon^{\prime}} \\
& =\frac{\tilde{\mu}_{m} a \pi\left[J_{1}\left(\tilde{\mu}_{m} a\right) Y_{0}\left(\tilde{\mu}_{m} a_{2}\right)-J_{0}\left(\tilde{\mu}_{m} a_{2}\right) Y_{1}\left(\tilde{\mu}_{m} a\right)\right] \kappa_{\mathrm{TiN}} \sinh \kappa_{\mathrm{TiN}} t}{2 \sigma_{\mathrm{TiN}}} \\
& -\frac{j Z_{0} \tilde{\mu}_{m}^{2} a \pi\left[J_{0}\left(\tilde{\mu}_{m} a_{2}\right) Y_{0}\left(\tilde{\mu}_{m} a\right)-J_{0}\left(\tilde{\mu}_{m} a\right) Y_{0}\left(\tilde{\mu}_{m} a_{2}\right)\right] \cosh \kappa_{\mathrm{TiN}} t}{2 k \beta \epsilon^{\prime}}, \\
& I_{0}^{(m)} \simeq \frac{j k \beta a \epsilon^{\prime}\left[I_{0}^{\prime}\left(\mu_{m}^{\epsilon} a\right) K_{0}^{\prime}\left(\mu_{m}^{\epsilon} a_{2}\right)-I_{0}^{\prime}\left(\mu_{m}^{\epsilon} a_{2}\right) K_{0}^{\prime}\left(\mu_{m}^{\epsilon} a\right)\right] \cosh \kappa_{\mathrm{TiN}} t}{Z_{0}} \\
& +\frac{\mu_{m}^{\epsilon} a \sigma_{\mathrm{TiN}}\left[I_{0}^{\prime}\left(\mu_{m}^{\epsilon} a_{2}\right) K_{0}\left(\mu_{m}^{\epsilon} a\right)-I_{0}\left(\mu_{m}^{\epsilon} a\right) K_{0}^{\prime}\left(\mu_{m}^{\epsilon} a_{2}\right)\right] \sinh \kappa_{\mathrm{TiN}} t}{\kappa_{\mathrm{TiN}}} \\
& =\frac{j k \beta a \epsilon^{\prime} \pi\left[J_{1}\left(\tilde{\mu}_{m} a\right) Y_{1}\left(\tilde{\mu}_{m} a_{2}\right)-J_{1}\left(\tilde{\mu}_{m} a_{2}\right) Y_{1}\left(\tilde{\mu}_{m} a\right)\right] \cosh \kappa_{\mathrm{TiN}} t}{2 Z_{0}} \\
& +\frac{\tilde{\mu}_{m} a \sigma_{\mathrm{TiN}} \pi\left[J_{1}\left(\tilde{\mu}_{m} a_{2}\right) Y_{0}\left(\tilde{\mu}_{m} a\right)-J_{0}\left(\tilde{\mu}_{m} a\right) Y_{1}\left(\tilde{\mu}_{m} a_{2}\right)\right] \sinh \kappa_{\mathrm{TiN}} t}{2 \kappa_{\mathrm{TiN}}}, \\
& K_{0}^{(m)} \simeq \frac{j k \beta a \epsilon^{\prime}\left[I_{0}^{\prime}\left(\mu_{m}^{e} a\right) K_{0}^{\prime}\left(\mu_{m}^{\epsilon} a_{2}\right)-I_{0}^{\prime}\left(\mu_{m}^{\epsilon} a_{2}\right) K_{0}^{\prime}\left(\mu_{m}^{\epsilon} a\right)\right] \kappa_{\mathrm{TiN}} \sinh \kappa_{\mathrm{TiN}} t}{\sigma_{\mathrm{TiN}} Z_{0}} \\
& +\mu_{m}^{\epsilon} a\left[I_{0}^{\prime}\left(\mu_{m}^{\epsilon} a_{2}\right) K_{0}\left(\mu_{m}^{\epsilon} a\right)-I_{0}\left(\mu_{m}^{\epsilon} a\right) K_{0}^{\prime}\left(\mu_{m}^{\epsilon} a_{2}\right)\right] \cosh \kappa_{\mathrm{TiN}} t \\
& =\frac{j k \beta a \epsilon^{\prime} \pi\left[J_{1}\left(\tilde{\mu}_{m} a\right) Y_{1}\left(\mu_{m}^{\epsilon} a_{2}\right)-J_{1}\left(\tilde{\mu}_{m} a_{2}\right) Y_{1}\left(\tilde{\mu}_{m} a\right)\right] \kappa_{\mathrm{TiN}} \sinh \kappa_{\mathrm{TiN}} t}{2 \sigma_{\mathrm{TiN}} Z_{0}} \\
& +\frac{\tilde{\mu}_{m} a \pi\left[J_{1}\left(\tilde{\mu}_{m} a_{2}\right) Y_{0}\left(\tilde{\mu}_{m} a\right)-J_{0}\left(\tilde{\mu}_{m} a\right) Y_{1}\left(\tilde{\mu}_{m} a_{2}\right)\right]}{2} \cosh \kappa_{\mathrm{TiN}} t
\end{aligned}
$$

where $\tilde{\mu}_{m}$ is given by Eq. (34) and

$$
\mu_{m}^{\epsilon}=\sqrt{\frac{m^{2} \pi^{2}}{4 w^{2}}-k^{2} \beta^{2} \epsilon^{\prime}},
$$

and $\kappa_{\mathrm{TiN}}$ is given by Eq. (39) in the text.

Specially for the $m=0$ case, Eqs. (C21)-(C24) become Eqs. (35) - (38) by assuming $\kappa_{\mathrm{TiN}} t \ll 1$ and $\left(a_{2}-a\right) / a \ll 1$.

\section{Dipole mode}

Following the procedure in the monopole case, let us, first consider the ceramic break made of a single material with conductivity $\sigma_{c}$, relative dielectric constant $\epsilon^{\prime}$ and relative permeability $\mu^{\prime}$. The fields in the ceramic break for dipole mode are expressed as 


$$
E_{z}=\left(\frac{1}{2 w} \tilde{\tilde{E}}_{z}^{(0)}+\frac{1}{w} \sum_{m=1}^{\infty} \cos \frac{m \pi(z+w)}{2 w} \tilde{\tilde{E}}_{z}^{(m)}\right) \cos \theta, \quad E_{\theta}=\frac{1}{w} \sum_{m=1}^{\infty} \sin \frac{m \pi(z+w)}{2 w} \tilde{\tilde{E}}_{\theta}^{(m)} \sin \theta
$$

$$
H_{\theta}=\left(\frac{1}{2 w} \tilde{\tilde{H}}_{\theta}^{(0)}+\frac{1}{w} \sum_{m=1}^{\infty} \cos \frac{m \pi(z+w)}{2 w} \tilde{\tilde{H}}_{\theta}^{(m)}\right) \cos \theta
$$

By solving Maxwell equations, the fields for $m=0$ on $\rho=a_{2}$ and those on $\rho=a$ are related as

$$
\left(\begin{array}{l}
\tilde{\tilde{E}}_{z}^{(0)}\left(a_{2}\right) \\
\tilde{\tilde{H}}_{\theta}^{(0)}\left(a_{2}\right)
\end{array}\right)=\left(\begin{array}{cc}
A_{1}^{(0)} & C_{1}^{(0)} \\
I_{1}^{(0)} & K_{1}^{(0)}
\end{array}\right)\left(\begin{array}{l}
\tilde{\tilde{E}}_{z}^{(0)}(a) \\
\tilde{\tilde{H}}_{\theta}^{(0)}(a)
\end{array}\right),
$$

$$
H_{z}=\frac{1}{w} \sum_{m=1}^{\infty} \sin \frac{m \pi(z+w)}{2 w} \tilde{\tilde{H}}_{z}^{(m)} \sin \theta
$$

where

$$
\begin{gathered}
A_{1}^{(0)}=\frac{\pi \kappa_{\mathrm{cer}} a\left[J_{1}\left(\kappa_{\mathrm{cer}} a_{2}\right) Y_{1}^{\prime}\left(\kappa_{\mathrm{cer}} a\right)-J_{1}^{\prime}\left(\kappa_{\mathrm{cer}} a\right) Y_{1}\left(\kappa_{\mathrm{cer}} a_{2}\right)\right]}{2}, \\
C_{1}^{(0)}=-\frac{j Z_{0} \pi k \beta \mu^{\prime} a\left[J_{1}\left(\kappa_{\mathrm{cer}} a_{2}\right) Y_{1}\left(\kappa_{\mathrm{cer}} a\right)-J_{1}\left(\kappa_{\mathrm{cer}} a\right) Y_{1}\left(\kappa_{\mathrm{cer}} a_{2}\right)\right]}{2}, \\
I_{1}^{(0)}=-j \frac{\pi a k \beta \tilde{\epsilon}\left[J_{1}^{\prime}\left(\kappa_{\mathrm{cer}} a_{2}\right) Y_{1}^{\prime}\left(\kappa_{\mathrm{cer}} a\right)-J_{1}^{\prime}\left(\kappa_{\mathrm{cer}} a\right) Y_{1}^{\prime}\left(\kappa_{\mathrm{cer}} a_{2}\right)\right]}{2 Z_{0}}, \\
K_{1}^{(0)}=\frac{\pi a k \beta \sqrt{\tilde{\epsilon} \mu^{\prime}}\left[J_{1}\left(\kappa_{\mathrm{cer}} a\right) Y_{1}^{\prime}\left(\kappa_{\mathrm{cer}} a_{2}\right)-J_{1}^{\prime}\left(\kappa_{\mathrm{cer}} a_{2}\right) Y_{1}\left(\kappa_{\mathrm{cer}} a\right)\right]}{2},
\end{gathered}
$$

and those for $m>0$ are done by

$$
\left(\begin{array}{c}
\tilde{\tilde{E}}_{z}^{(m)}\left(a_{2}\right) \\
\tilde{\tilde{H}}_{z}^{(m)}\left(a_{2}\right) \\
\tilde{\tilde{H}}_{\theta}^{(m)}\left(a_{2}\right) \\
\tilde{\tilde{E}}_{\theta}^{(m)}\left(a_{2}\right)
\end{array}\right)=\left(\begin{array}{cccc}
A_{1}^{(m)} & B_{1}^{(m)} & C_{1}^{(m)} & D_{1}^{(m)} \\
E_{1}^{(m)} & F_{1}^{(m)} & G_{1}^{(m)} & H_{1}^{(m)} \\
I_{1}^{(m)} & J_{1}^{(m)} & K_{1}^{(m)} & L_{1}^{(m)} \\
M_{1}^{(m)} & N_{1}^{(m)} & O_{1}^{(m)} & P_{1}^{(m)}
\end{array}\right)\left(\begin{array}{c}
\tilde{\tilde{E}}_{z}^{(m)}(a) \\
\tilde{\tilde{H}}_{z}^{(m)}(a) \\
\tilde{\tilde{H}}_{\theta}^{(m)}(a) \\
\tilde{\tilde{E}}_{\theta}^{(m)}(a)
\end{array}\right),
$$

where

$$
A_{1}^{(m)}=\mu_{m} a\left[I_{1}^{\prime}\left(\mu_{m} a\right) K_{1}\left(\mu_{m} a_{2}\right)-I_{1}\left(\mu_{m} a_{2}\right) K_{1}^{\prime}\left(\mu_{m} a\right)\right]
$$

$$
B_{1}^{(m)}=-\frac{j \pi m Z_{0}\left[I_{1}\left(\mu_{m} a_{2}\right) K_{1}\left(\mu_{m} a\right)-I_{1}\left(\mu_{m} a\right) K_{1}\left(\mu_{m} a_{2}\right)\right]}{g k \beta \tilde{\epsilon}},
$$$$
F_{1}^{(m)}=\mu_{m} a\left[I_{1}^{\prime}\left(\mu_{m} a\right) K_{1}\left(\mu_{m} a_{2}\right)-I_{1}\left(\mu_{m} a_{2}\right) K_{1}^{\prime}\left(\mu_{m} a\right)\right],
$$$$
E_{1}^{(m)}=\frac{j \pi m\left[I_{1}\left(\mu_{m} a_{2}\right) K_{1}\left(\mu_{m} a\right)-I_{1}\left(\mu_{m} a\right) K_{1}\left(\mu_{m} a_{2}\right)\right]}{g k Z_{0} \beta \mu^{\prime}},
$$

$$
C_{1}^{(m)}=-\frac{j Z_{0} \mu_{m}^{2} a\left[I_{1}\left(\mu_{m} a_{2}\right) K_{1}\left(\mu_{m} a\right)-I_{1}\left(\mu_{m} a\right) K_{1}\left(\mu_{m} a_{2}\right)\right]}{k \beta \tilde{\epsilon}},
$$

$$
G_{1}^{(m)}=0,
$$

$$
D_{1}^{(m)}=0,
$$

$$
H_{1}^{(m)}=\frac{j \mu_{m}^{2} a\left[I_{1}\left(\mu_{m} a_{2}\right) K_{1}\left(\mu_{m} a\right)-I_{1}\left(\mu_{m} a\right) K_{1}\left(\mu_{m} a_{2}\right)\right]}{k Z_{0} \beta \mu^{\prime}},
$$




$$
\begin{aligned}
I_{1}^{(m)}= & \frac{j k \beta a \tilde{\epsilon}\left[I_{1}^{\prime}\left(\mu_{m} a\right) K_{1}^{\prime}\left(\mu_{m} a_{2}\right)-I_{1}^{\prime}\left(\mu_{m} a_{2}\right) K_{1}^{\prime}\left(\mu_{m} a\right)\right]}{Z_{0}} \\
& -\frac{j \pi^{2} m^{2}\left[I_{1}\left(\mu_{m} a_{2}\right) K_{1}\left(\mu_{m} a\right)-I_{1}\left(\mu_{m} a\right) K_{1}\left(\mu_{m} a_{2}\right)\right]}{a_{2} g^{2} k \beta Z_{0} \mu^{\prime} \mu_{m}^{2}},
\end{aligned}
$$

$$
\begin{aligned}
J_{1}^{(m)}= & -\frac{\pi m a\left[I_{1}^{\prime}\left(\mu_{m} a\right) K_{1}\left(\mu_{m} a_{2}\right)-I_{1}\left(\mu_{m} a_{2}\right) K_{1}^{\prime}\left(\mu_{m} a\right)\right]}{a_{2} g \mu_{m}} \\
& -\frac{\pi m\left[I_{1}\left(\mu_{m} a\right) K_{1}^{\prime}\left(\mu_{m} a_{2}\right)-I_{1}^{\prime}\left(\mu_{m} a_{2}\right) K_{1}\left(\mu_{m} a\right)\right]}{g \mu_{m}},
\end{aligned}
$$

$$
K_{1}^{(m)}=\mu_{m} a\left[I_{1}^{\prime}\left(\mu_{m} a_{2}\right) K_{1}\left(\mu_{m} a\right)-I_{1}\left(\mu_{m} a\right) K_{1}^{\prime}\left(\mu_{m} a_{2}\right)\right],
$$

$$
L_{1}^{(m)}=-\frac{j \pi m a\left[I_{1}\left(\mu_{m} a_{2}\right) K_{1}\left(\mu_{m} a\right)-I_{1}\left(\mu_{m} a\right) K_{1}\left(\mu_{m} a_{2}\right)\right]}{a_{2} g k \beta Z_{0} \mu^{\prime}},
$$

$$
\begin{aligned}
M_{1}^{(m)}= & \frac{\pi m a\left[I_{1}\left(\mu_{m} a_{2}\right) K_{1}^{\prime}\left(\mu_{m} a\right)-I_{1}^{\prime}\left(\mu_{m} a\right) K_{1}\left(\mu_{m} a_{2}\right)\right]}{g \mu_{m} a_{2}} \\
& -\frac{\pi m\left[I_{1}\left(\mu_{m} a\right) K_{1}^{\prime}\left(\mu_{m} a_{2}\right)-I_{1}^{\prime}\left(\mu_{m} a_{2}\right) K_{1}\left(\mu_{m} a\right)\right]}{g \mu_{m}},
\end{aligned}
$$

$$
\begin{array}{r}
N_{1}^{(m)}=j Z_{0} k \beta \mu^{\prime} a\left[I_{1}^{\prime}\left(\mu_{m} a_{2}\right) K_{1}^{\prime}\left(\mu_{m} a\right)-I_{1}^{\prime}\left(\mu_{m} a\right) K_{1}^{\prime}\left(\mu_{m} a_{2}\right)\right] \\
-\frac{j Z_{0} \pi^{2} m^{2}\left[I_{1}\left(\mu_{m} a\right) K_{1}\left(\mu_{m} a_{2}\right)-I_{1}\left(\mu_{m} a_{2}\right) K_{1}\left(\mu_{m} a\right)\right]}{a_{2} g^{2} k \beta \tilde{\epsilon} \mu_{m}^{2}}, \quad(\mathrm{C} 49) \\
O_{1}^{(m)}=\frac{j \pi m Z_{0} a\left[I_{1}\left(\mu_{m} a_{2}\right) K_{1}\left(\mu_{m} a\right)-I_{1}\left(\mu_{m} a\right) K_{1}\left(\mu_{m} a_{2}\right)\right]}{a_{2} g k \beta \tilde{\epsilon}},
\end{array}
$$

$$
P_{1}^{(m)}=\mu_{m} a\left[I_{1}^{\prime}\left(\mu_{m} a_{2}\right) K_{1}\left(\mu_{m} a\right)-I_{1}\left(\mu_{m} a\right) K_{1}^{\prime}\left(\mu_{m} a_{2}\right)\right] .
$$

If the ceramic break is made of a ceramic with $\epsilon^{\prime}$ where the inner surface is coated with thin TiN with the thickness $t$ and the conductivity $\sigma_{\mathrm{TiN}}$, the transfer coefficients for $m=0$ are calculated as

$$
\begin{aligned}
& A_{1}^{(0)} \simeq \frac{\pi \kappa_{\mathrm{cer}}^{\epsilon} a\left[J_{1}\left(\kappa_{\mathrm{cer}}^{\epsilon} a_{2}\right) Y_{1}^{\prime}\left(\kappa_{\mathrm{cer}}^{\epsilon} a\right)-J_{1}^{\prime}\left(\kappa_{\mathrm{cer}}^{\epsilon} a\right) Y_{1}\left(\kappa_{\mathrm{cer}}^{\epsilon} a_{2}\right)\right] \cosh \kappa_{\mathrm{TiN}} t}{2} \\
& +\left[\frac{\kappa_{\mathrm{cer}}^{\epsilon}\left[J_{1}\left(\kappa_{\mathrm{cer}}^{\epsilon} a_{2}\right) Y_{1}^{\prime}\left(\kappa_{\mathrm{cer}}^{\epsilon} a\right)-J_{1}^{\prime}\left(\kappa_{\mathrm{cer}}^{\epsilon} a\right) Y_{1}\left(\kappa_{\mathrm{cer}}^{\epsilon} a_{2}\right)\right]}{2}\right. \\
& \left.-j \sigma_{c} Z_{0} k \beta a\left[J_{1}\left(\kappa_{\mathrm{cer}}^{\epsilon} a_{2}\right) Y_{1}\left(\kappa_{\mathrm{cer}}^{\epsilon} a\right)-J_{1}\left(\kappa_{\mathrm{cer}}^{\epsilon} a\right) Y_{1}\left(\kappa_{\mathrm{cer}}^{\epsilon} a_{2}\right)\right]\right] \frac{\pi \sinh \kappa_{\mathrm{TiN}} t}{2 \kappa_{\mathrm{TiN}}}, \\
& C_{1}^{(0)} \simeq\left[\frac{\kappa_{\mathrm{cer}}^{\epsilon} a \kappa_{\mathrm{TiN}}\left[J_{1}\left(\kappa_{\mathrm{cer}}^{\epsilon} a_{2}\right) Y_{1}^{\prime}\left(\kappa_{\mathrm{cer}}^{\epsilon} a\right)-J_{1}^{\prime}\left(\kappa_{\mathrm{cer}}^{\epsilon} a\right) Y_{1}\left(\kappa_{\mathrm{cer}}^{\epsilon} a_{2}\right)\right]}{\sigma_{c}}\right. \\
& \left.+\frac{j Z_{0} k \beta\left[J_{1}\left(\kappa_{\mathrm{cer}}^{\epsilon} a_{2}\right) Y_{1}\left(\kappa_{\mathrm{cer}}^{\epsilon} a\right)-J_{1}\left(\kappa_{\mathrm{cer}}^{\epsilon} a\right) Y_{1}\left(\kappa_{\mathrm{cer}}^{\epsilon} a_{2}\right)\right]}{2 \kappa_{\mathrm{TiN}}}\right] \frac{\pi \sinh \kappa_{\mathrm{TiN}} t}{2} \\
& -\frac{j Z_{0} \pi k \beta a\left[J_{1}\left(\kappa_{\mathrm{cer}}^{\epsilon} a_{2}\right) Y_{1}\left(\kappa_{\mathrm{cer}}^{\epsilon} a\right)-J_{1}\left(\kappa_{\mathrm{cer}}^{\epsilon} a\right) Y_{1}\left(\kappa_{\mathrm{cer}}^{\epsilon} a_{2}\right)\right] \cosh \kappa_{\mathrm{TiN}} t}{2}, \\
& I_{1}^{(0)} \simeq-j \frac{\pi a k \beta \epsilon^{\prime}\left[J_{1}^{\prime}\left(\kappa_{\mathrm{cer}}^{\epsilon} a_{2}\right) Y_{1}^{\prime}\left(\kappa_{\mathrm{cer}}^{\epsilon} a\right)-J_{1}^{\prime}\left(\kappa_{\mathrm{cer}}^{\epsilon} a\right) Y_{1}^{\prime}\left(\kappa_{\mathrm{cer}}^{\epsilon} a_{2}\right)\right] \cosh \kappa_{\mathrm{TiN}} t}{2 Z_{0}} \\
& +\left[-j \frac{\epsilon^{\prime}\left[J_{1}^{\prime}\left(\kappa_{\mathrm{cer}}^{\epsilon} a_{2}\right) Y_{1}^{\prime}\left(\kappa_{\mathrm{cer}}^{\epsilon} a\right)-J_{1}^{\prime}\left(\kappa_{\mathrm{cer}}^{\epsilon} a\right) Y_{1}^{\prime}\left(\kappa_{\mathrm{cer}}^{\epsilon} a_{2}\right)\right]}{2 Z_{0}}\right. \\
& \left.+\sigma_{c} a \sqrt{\epsilon^{\prime}}\left[J_{1}\left(\kappa_{\mathrm{cer}}^{\epsilon} a\right) Y_{1}^{\prime}\left(\kappa_{\mathrm{cer}}^{\epsilon} a_{2}\right)-J_{1}^{\prime}\left(\kappa_{\mathrm{cer}}^{\epsilon} a_{2}\right) Y_{1}\left(\kappa_{\mathrm{cer}}^{\epsilon} a\right)\right]\right] \frac{\pi k \beta \sinh \kappa_{\mathrm{TiN}} t}{2 \kappa_{\mathrm{TiN}}},
\end{aligned}
$$




$$
\begin{aligned}
K_{1}^{(0)} \simeq & \frac{\pi a k \beta \sqrt{\epsilon^{\prime}}\left[J_{1}\left(\kappa_{\mathrm{cer}}^{\epsilon} a\right) Y_{1}^{\prime}\left(\kappa_{\mathrm{cer}}^{\epsilon} a_{2}\right)-J_{1}^{\prime}\left(\kappa_{\mathrm{cer}}^{\epsilon} a_{2}\right) Y_{1}\left(\kappa_{\mathrm{cer}}^{\epsilon} a\right)\right] \cosh \kappa_{\mathrm{TiN}} t}{2} \\
& +\left[-j \frac{a \epsilon^{\prime} \kappa_{\mathrm{TiN}}\left[J_{1}^{\prime}\left(\kappa_{\mathrm{cer}}^{\epsilon} a_{2}\right) Y_{1}^{\prime}\left(\kappa_{\mathrm{cer}}^{\epsilon} a\right)-J_{1}^{\prime}\left(\kappa_{\mathrm{cer}}^{\epsilon} a\right) Y_{1}^{\prime}\left(\kappa_{\mathrm{cer}}^{\epsilon} a_{2}\right)\right]}{Z_{0} \sigma_{c}}\right. \\
& \left.-\frac{\sqrt{\epsilon^{\prime}}\left[J_{1}\left(\kappa_{\mathrm{cer}}^{\epsilon} a\right) Y_{1}^{\prime}\left(\kappa_{\mathrm{cer}}^{\epsilon} a_{2}\right)-J_{1}^{\prime}\left(\kappa_{\mathrm{cer}}^{\epsilon} a_{2}\right) Y_{1}\left(\kappa_{\mathrm{cer}}^{\epsilon} a\right)\right]}{2 \kappa_{\mathrm{TiN}}}\right] \frac{\pi k \beta \sinh \kappa_{\mathrm{TiN}} t}{2},
\end{aligned}
$$

where

$$
\kappa_{\mathrm{cer}}^{\epsilon}=\sqrt{k^{2} \beta^{2} \epsilon^{\prime}},
$$

and $\kappa_{\mathrm{TiN}}$ is described in Eq. (39) in the text.

For $m>0$, the transfer coefficients $A_{1}^{(m)} \cdots P_{1}^{(m)}$ for the dipole mode are described as

$$
\begin{gathered}
A_{1}^{(m)}=A_{\epsilon}^{(m)} A_{t}^{(m)}+B_{\epsilon}^{(m)} E_{t}^{(m)}+C_{\epsilon}^{(m)} I_{t}^{(m)}, \\
B_{1}^{(m)}=A_{\epsilon}^{(m)} B_{t}^{(m)}+B_{\epsilon}^{(m)} F_{t}^{(m)}+C_{\epsilon}^{(m)} J_{t}^{(m)}, \\
C_{1}^{(m)}=A_{\epsilon}^{(m)} C_{t}^{(m)}+C_{\epsilon}^{(m)} K_{t}^{(m)}, \\
D_{1}^{(m)}=B_{\epsilon}^{(m)} H_{t}^{(m)}+C_{\epsilon}^{(m)} L_{t}^{(m)}, \\
E_{1}^{(m)}=A_{t}^{(m)} E_{\epsilon}^{(m)}+E_{t}^{(m)} F_{\epsilon}^{(m)}+H_{\epsilon}^{(m)} M_{t}^{(m)}, \\
F_{1}^{(m)}=B_{t}^{(m)} E_{\epsilon}^{(m)}+F_{\epsilon}^{(m)} F_{t}^{(m)}+H_{\epsilon}^{(m)} N_{t}^{(m)}, \\
G_{1}^{(m)}=C_{t}^{(m)} E_{\epsilon}^{(m)}+H_{\epsilon}^{(m)} O_{t}^{(m)}, \\
H_{1}^{(m)}=F_{\epsilon}^{(m)} H_{t}^{(m)}+H_{\epsilon}^{(m)} P_{t}^{(m)},
\end{gathered}
$$

$$
I_{1}^{(m)}=A_{t}^{(m)} \boldsymbol{I}_{\epsilon}^{(m)}+E_{t}^{(m)} \boldsymbol{J}_{\epsilon}^{(m)}+\boldsymbol{I}_{t}^{(m)} \boldsymbol{K}_{\epsilon}^{(m)}+L_{\epsilon}^{(m)} M_{t}^{(m)},
$$

$$
\boldsymbol{J}_{1}^{(m)}=B_{t}^{(m)} \boldsymbol{I}_{\epsilon}^{(m)}+F_{t}^{(m)} \boldsymbol{J}_{\epsilon}^{(m)}+\boldsymbol{J}_{t}^{(m)} \boldsymbol{K}_{\epsilon}^{(m)}+L_{\epsilon}^{(m)} N_{t}^{(m)},
$$

$$
K_{1}^{(m)}=C_{t}^{(m)} \boldsymbol{I}_{\epsilon}^{(m)}+K_{\epsilon}^{(m)} K_{t}^{(m)}+L_{\epsilon}^{(m)} O_{t}^{(m)},
$$$$
L_{1}^{(m)}=H_{t}^{(m)} \boldsymbol{J}_{\epsilon}^{(m)}+K_{\epsilon}^{(m)} L_{t}^{(m)}+L_{\epsilon}^{(m)} P_{t}^{(m)},
$$

$$
M_{1}^{(m)}=A_{t}^{(m)} M_{\epsilon}^{(m)}+E_{t}^{(m)} N_{\epsilon}^{(m)}+I_{t}^{(m)} O_{\epsilon}^{(m)}+M_{t}^{(m)} P_{\epsilon}^{(m)},
$$

$$
N_{1}^{(m)}=B_{t}^{(m)} M_{\epsilon}^{(m)}+F_{t}^{(m)} N_{\epsilon}^{(m)}+J_{t}^{(m)} O_{\epsilon}^{(m)}+N_{t}^{(m)} P_{\epsilon}^{(m)},
$$

$$
\begin{aligned}
& O_{1}^{(m)}=C_{t}^{(m)} M_{\epsilon}^{(m)}+K_{t}^{(m)} O_{\epsilon}^{(m)}+O_{t}^{(m)} P_{\epsilon}^{(m)}, \\
& P_{1}^{(m)}=H_{t}^{(m)} N_{\epsilon}^{(m)}+L_{t}^{(m)} O_{\epsilon}^{(m)}+P_{\epsilon}^{(m)} P_{t}^{(m)}
\end{aligned}
$$

where $A_{\epsilon}^{(m)}, \ldots, P_{\epsilon}^{(m)}$ are given by replacing $\mu_{m}, \tilde{\epsilon}$ and $\mu^{\prime}$ in Eqs. (C36)-(C51) by $\mu_{m}^{\epsilon}$ [defined in Eq. (C25)], $\epsilon^{\prime}$ (dielectric constant of ceramic) and $\mu^{\prime}=1$, respectively. The coefficients $A_{t}^{(m)}, \ldots, P_{t}^{(m)}$ are approximated as

$$
\begin{aligned}
& A_{t}^{(m)}=\cosh \kappa_{\mathrm{TiN}} t+\frac{\sinh \kappa_{\mathrm{TiN}} t}{2 \kappa_{\mathrm{TiN}} a}, \\
& B_{t}^{(m)}=\frac{\pi m}{g \sigma_{c}} \frac{\sinh \kappa_{\mathrm{TiN}} t}{\kappa_{\mathrm{TiN}} a}, \\
& C_{t}^{(m)}=\frac{\kappa_{\mathrm{TiN}}}{\sigma_{c}} \sinh \kappa_{\mathrm{TiN}} t, \\
& D_{t}^{(m)}=0, \\
& E_{t}^{(m)}=\frac{j \pi m}{g k Z_{0} \beta} \frac{\sinh \kappa_{\mathrm{TiN}} t}{\kappa_{\mathrm{TiN}} a}, \\
& F_{t}^{(m)}=\cosh \kappa_{\mathrm{TiN}} t+\frac{\sinh \kappa_{\mathrm{TiN}} t}{2 \kappa_{\mathrm{TiN}} a}, \\
& G_{t}^{(m)}=0, \\
& H_{t}^{(m)}=\frac{j \kappa_{\mathrm{TiN}}}{k Z_{0} \beta} \sinh \kappa_{\mathrm{TiN}} t, \\
& I_{t}^{(m)}=\left(\sigma_{c}-\frac{\pi^{2} m^{2}}{a^{2} g^{2} k^{2} \beta^{2} \sigma_{c} Z_{0}^{2}}\right) \frac{\sinh \kappa_{\mathrm{TiN}} t}{\kappa_{\mathrm{TiN}}}, \\
& J_{t}^{(m)}=\frac{\pi m t}{g j k \beta Z_{0} \sigma_{c} a^{2}} \cosh \kappa_{\mathrm{TiN}} t, \\
& K_{t}^{(m)}=\cosh \kappa_{\mathrm{TiN}} t-\frac{\sinh \kappa_{\mathrm{TiN}} t}{2 \kappa_{\mathrm{TiN}} a}, \\
& L_{t}^{(m)}=-\frac{j \pi m \sinh \kappa_{\mathrm{TiN}} t}{a g k \beta Z_{0} \kappa_{\mathrm{TiN}}}, \\
& M_{t}^{(m)}=\frac{\pi m t}{g j k \beta Z_{0} \sigma_{c} a^{2}} \cosh \kappa_{\mathrm{TiN}} t,
\end{aligned}
$$




$$
\begin{gathered}
N_{t}^{(m)}=\left(-j Z_{0} k \beta \mu^{\prime}+\frac{j \pi^{2} m^{2}}{g^{2} k \beta \sigma_{c}^{2} Z_{0} a^{2}}\right) \frac{\sinh \kappa_{\mathrm{TiN}} t}{\kappa_{\mathrm{TiN}}}, \\
O_{t}^{(m)}=-\frac{\pi m \sinh \kappa_{\mathrm{TiN}} t}{(a+t) g \sigma_{c} \kappa_{\mathrm{TiN}}}, \\
P_{t}^{(m)}=\cosh \kappa_{\mathrm{TiN}} t-\frac{\sinh \kappa_{\mathrm{TiN}} t}{2 \kappa_{\mathrm{TiN}} a}
\end{gathered}
$$

respectively.

\section{APPENDIX D: CONCRETE EXPRESSIONS OF}

$\left\langle\left\langle Y_{-2}^{(n)}\right\rangle\right\rangle_{m}^{s},\left\langle\left\langle Y_{-1}\right\rangle\right\rangle_{m}^{s},\left\langle\left\langle Y_{0}^{(n)}\right\rangle\right\rangle_{m}^{s},\left\langle\left\langle Y_{0}^{(n)}\right\rangle\right\rangle_{m}^{s},\left\langle\left\langle Y_{0}^{(n)}\right\rangle\right\rangle_{m}^{s}$, $\left\langle Y_{1}^{(n)}-Y_{4}^{(n)} / k^{2} \beta^{2} a^{2}\right\rangle,\left\langle\left\langle Y_{1}^{(n)}-Y_{4}^{(n)} / k^{2} \beta^{2} a^{2}\right\rangle\right\rangle_{m},\left\langle{Y_{4}^{\prime}}_{4}^{(n)}\right\rangle$, $\left\langle\left\langle Y_{4}^{\prime(n)}\right\rangle_{m},\left\langle Y_{2}-Y_{3} / k^{2} \beta^{2} a^{2}\right\rangle,\left\langle\left\langle Y_{2}-Y_{3} / k^{2} \beta^{2} a^{2}\right\rangle\right\rangle_{m}\right.$, $\left\langle Y_{5}-Y_{6} / k^{2} \beta^{2} a_{2}^{2}\right\rangle,\left\langle\left\langle Y_{5}-Y_{6} / k^{2} \beta^{2} a_{2}^{2}\right\rangle\right\rangle_{m}$, $\left\langle Y_{7}^{(n)}-Y_{8}^{(n)} / k^{2} \beta^{2} a_{2}^{2}\right\rangle,\left\langle\left\langle Y_{7}^{(n)}(z)-Y_{8}^{(n)}(z) / k^{2} \beta^{2} a_{2}^{2}\right\rangle\right\rangle_{m}$, $\left\langle Y_{9}^{(n)}\right\rangle,\left\langle\left\langle Y_{9}^{(n)}\right\rangle\right\rangle_{m},\left\langle\left\langle Y_{10}(z)\right\rangle\right\rangle_{m}^{s},\left\langle\left\langle Y_{11}^{(n)}\right\rangle\right\rangle_{m}^{s}$ AND $\left\langle\left\langle Y_{12}^{(n)}\right\rangle_{m}^{s}\right.$

The brackets $\langle\cdots\rangle,\langle\langle\cdots\rangle\rangle_{m}$ and $\langle\langle\cdots\rangle\rangle_{m}^{s}$ are defined by Eqs. (A18), (A19) and (A49) in

Appendix A, respectively. Since the functions $Y_{-2}^{(n)}(z)$, $Y_{-1}(z), Y_{0}^{(n)}(z), Y_{1}^{(n)}(z), Y_{2}(z), Y_{3}(z), Y_{4}^{(n)}(z), Y_{4}^{\prime(n)}(z)$ are provided by Eqs. (A41)-(A48) in Appendix A 2, respectively, formal expressions of $\left\langle\left\langle Y_{-2}^{(n)}\right\rangle\right\rangle_{m}^{s},\left\langle\left\langle Y_{-1}\right\rangle\right\rangle_{m}^{s},\left\langle\left\langle Y_{0}^{(n)} o\right\rangle_{m}^{s}\right.$, $\left\langle\left\langle Y_{0}^{(n)}\right\rangle\right\rangle_{m}^{s}, \quad\left\langle\left\langle Y_{0}^{(n)}\right\rangle\right\rangle_{m}^{s}, \quad\left\langle Y_{1}^{(n)}-Y_{4}^{(n)} / k^{2} \beta^{2} a^{2}\right\rangle, \quad\left\langle\left\langle Y_{1}^{(n)}-Y_{4}^{(n)} /\right.\right.$ $\left.\left.k^{2} \beta^{2} a^{2}\right\rangle\right\rangle_{m},\left\langle Y_{4}^{\prime(n)}\right\rangle,\left\langle\left\langle Y_{4}^{\prime(n)}\right\rangle\right\rangle_{m},\left\langle Y_{2}-Y_{3} / k^{2} \beta^{2} a^{2}\right\rangle,\left\langle\left\langle Y_{2}-Y_{3}\right|\right.$ $\left.\left.k^{2} \beta^{2} a^{2}\right\rangle\right\rangle_{m}, \quad\left\langle Y_{5}-Y_{6} / k^{2} \beta^{2} a_{2}^{2}\right\rangle, \quad\left\langle\left\langle Y_{5}-Y_{6} / k^{2} \beta^{2} a_{2}^{2}\right\rangle\right\rangle_{m}$, $\left\langle Y_{7}^{(n)}-Y_{8}^{(n)} / k^{2} \beta^{2} a_{2}^{2}\right\rangle,\left\langle\left\langle Y_{7}^{(n)}(z)-Y_{8}^{(n)}(z) / k^{2} \beta^{2} a_{2}^{2}\right\rangle\right\rangle_{m},\left\langle Y_{9}^{(n)}\right\rangle$, $\left\langle\left\langle Y_{9}^{(n)}\right\rangle\right\rangle_{m},\left\langle\left\langle Y_{10}(z)\right\rangle\right\rangle_{m}^{s},\left\langle\left\langle Y_{11}^{(n)}\right\rangle\right\rangle_{m}^{s}$ and $\left\langle\left\langle Y_{12}^{(n)}\right\rangle\right\rangle_{m}^{s}$ are obtained by substituting them into Eqs. (A18), (A19) and (A49). The $z$ integrations are simply done. The remained $h$ integrations can be performed by using the expansion formulas [10] for Bessel functions

$$
\begin{gathered}
\frac{J_{1}^{\prime}(z)}{J_{1}(z)}=\frac{1}{z}+\sum_{k=1}^{\infty} \frac{2 z}{z^{2}-j_{1, k}^{2}}, \\
\frac{J_{1}(z)}{z J_{1}^{\prime}(z)}=\sum_{k=1}^{\infty} \frac{2}{z^{2}-j_{1, k}^{\prime 2}} \frac{J_{1}\left(j_{1, k}^{\prime}\right)}{J_{1}^{\prime \prime}\left(j_{1, k}^{\prime}\right)},
\end{gathered}
$$

$$
\frac{1}{\sqrt{z}} \frac{H_{1}^{(2)}(\sqrt{z})}{H_{1}^{\prime(2)}(\sqrt{z})}=\frac{2 H_{1}^{(2)}\left(h_{1,0}^{\prime}\right)}{\left(z-h_{1,0}^{\prime 2}\right) H_{1}^{\prime(2)}\left(h_{1,0}^{\prime}\right)}+\int_{0}^{\infty} d \zeta \frac{2}{\pi^{2} \zeta(\zeta+z) H_{1}^{\prime(1)}\left(e^{\frac{\pi}{2} j} \sqrt{\zeta}\right) H_{1}^{\prime(2)}\left(e^{\frac{\pi}{2} j} \sqrt{\zeta}\right)},
$$

where $j_{n, s}$ are the $s$ th zeros of $J_{n}(z), j_{1, s}^{\prime}$ are the $s$ th zeros of $J_{1}{ }^{\prime}(z), h_{1,0}^{\prime}=0.501184+j 0.643545$ is the 0 th zero of $H_{1}^{\prime(2)}(z)$ (the differential of the Hankel function of the second kind), $H_{1}^{(1)}(z)$ is the Hankel function of the first kind and the prime denotes the differential by its argument $z$. As in Appendix B, the integration of the Bessel functions can be again done by simply picking up residues in the complex plane $h$. The path of integration should be chosen to be below the poles for $h<0$ and above the poles for $h>0$.

Finally, we obtain the following expressions:

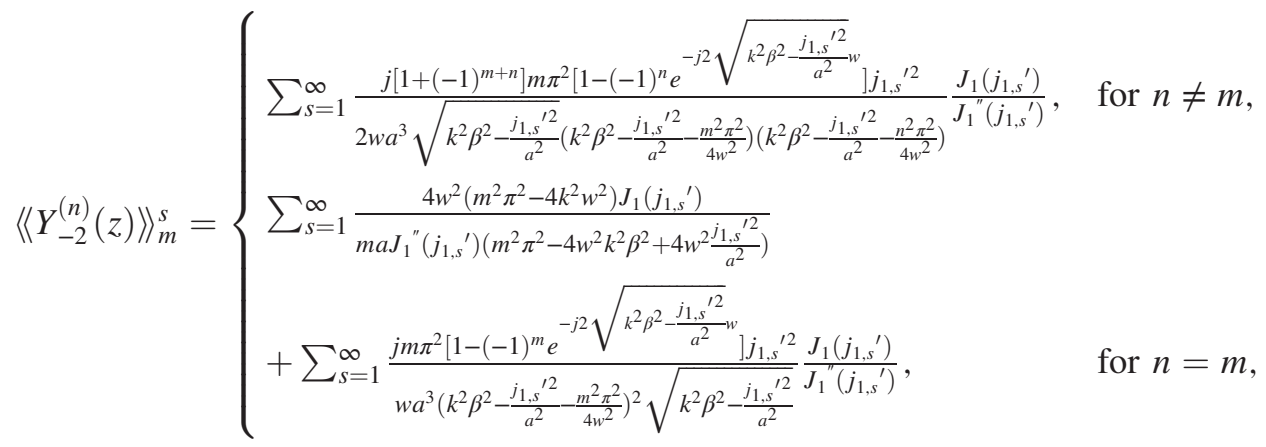

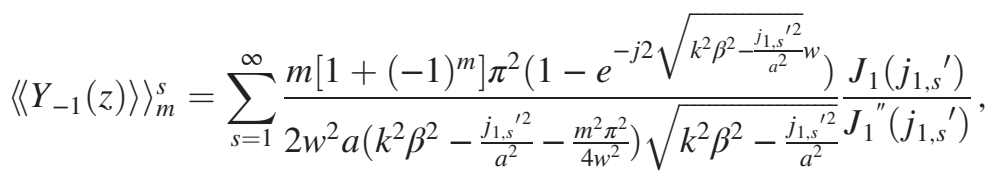




$$
\begin{aligned}
& \left\langle Y_{0}^{(n)}(z)\right\rangle_{m}^{s}= \begin{cases}\sum_{s=1}^{\infty} \frac{j\left[1+(-1)^{m+n}\right] m \pi^{2} \sqrt{k^{2} \beta^{2}-\frac{j_{1, s}^{\prime 2}}{a^{2}}}\left[1-(-1)^{m} e^{-j 2} \sqrt{k^{2} \beta^{2}-\frac{j_{1, s}^{\prime 2}}{a^{2} w}}\right] J_{1}\left(j_{1, s}^{\prime}\right)}{2 w a\left(k^{2} \beta^{2}-\frac{j_{1, s}^{\prime 2}}{a^{2}}-\frac{m^{2} \pi^{2}}{4 w^{2}}\right)\left(k^{2} \beta^{2}-\frac{j_{1, s}^{\prime 2}}{a^{2}}-\frac{n^{2} \pi^{2}}{4 w^{2}}\right)}, & \text { for } n \neq m, \\
\sum_{1}^{\prime \prime}\left(j_{1, s}^{\prime}\right) & \\
-\sum_{S=1}^{\infty} \frac{j m \pi^{2} \sqrt{k^{2} \beta^{2}-\frac{j_{1, s}^{\prime 2}}{a^{2}}}\left[1-(-1)^{m} e^{-j 2} \sqrt{k^{2} \beta^{2}-\frac{j_{1, s}^{\prime 2}}{a^{2}}}\right] J_{1}\left(j_{1, s}^{\prime}\right)}{a\left(\frac{m^{2} \pi^{2}}{4 w^{2}}-k^{2} \beta^{2}+\frac{j_{1, s}^{\prime 2}}{a^{2}}\right) J_{1}^{\prime \prime}\left(j_{1, s}^{\prime}\right)}, & \text { for } n=m, \\
J_{1}^{\prime \prime}\left(j_{1, s}^{\prime}\right) & \end{cases} \\
& \left\langle Y_{1}^{(n)}(z)-\frac{Y_{4}^{(n)}(z)}{k^{2} \beta^{2} a^{2}}\right\rangle=-\frac{\pi\left[1+(-1)^{n}\right]\left(1-e^{-j 2 k \beta w}\right)}{4 k \beta w a\left(k^{2} \beta^{2}-\frac{n^{2} \pi^{2}}{4 w^{2}}\right)} \\
& -\sum_{s=1}^{\infty} \frac{\pi\left[1+(-1)^{n}\right]\left(1-e^{\left.-j 2 \sqrt{k^{2} \beta^{2}-\frac{j_{1, s}^{2}}{a^{2}} w}\right)}\right.}{2 w a\left(k^{2} \beta^{2}-\frac{j_{1, s}^{2}}{a^{2}}-\frac{n^{2} \pi^{2}}{4 w^{2}}\right) \sqrt{k^{2} \beta^{2}-\frac{j_{1, s}^{2}}{a^{2}}}} \\
& -\sum_{s=1}^{\infty} \frac{\pi\left[1+(-1)^{n}\right]\left(1-e^{-j 2 k \beta w}\right)}{2 k \beta a w\left(k^{2} \beta^{2}-\frac{n^{2} \pi^{2}}{4 w^{2}} j_{1, s}^{\prime 2}\right.} \frac{J_{1}\left(j_{1, s}^{\prime}\right)}{J_{1}^{\prime \prime}\left(j_{1, s}^{\prime}\right)}
\end{aligned}
$$

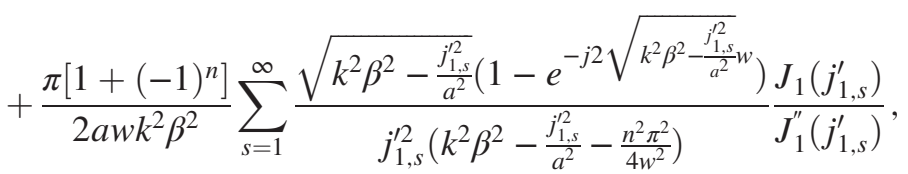

$$
\begin{aligned}
& \left\{\begin{array}{l}
-\frac{\left[1+(-1)^{m+n}\right] \pi k \beta\left[1-(-1)^{m} e^{-j 2 k \beta w}\right]}{2 a\left(k^{2} \beta^{2}-\frac{m^{2} \pi^{2}}{4 w^{2}}\right)\left(k^{2} \beta^{2}-\frac{n^{2} \pi^{2}}{4 w^{2}}\right)} \\
-\sum_{S=1}^{\infty} \frac{\left[1+(-1)^{n+m}\right] \pi \sqrt{k^{2} \beta^{2}-\frac{j_{1, s}^{2}}{a^{2}}}\left[1-(-1)^{m} e^{-j 2} \sqrt{k^{2} \beta^{2}-\frac{j_{1, s}^{2}}{a^{2}} w}\right]}{a\left(k^{2} \beta^{2}-\frac{j_{1, s}^{2}}{a^{2}}-\frac{m^{2} \pi^{2}}{4 w^{2}}\right)\left(k^{2} \beta^{2}-\frac{j_{1, s}^{2}}{a^{2}}-\frac{n^{2} \pi^{2}}{4 w^{2}}\right)} \\
-\frac{\left[1+(-1)^{m+n}\right] \pi k \beta\left[1-(-1)^{n} e^{-j 2 k \beta w}\right]}{a\left(k^{2} \beta^{2}-\frac{m^{2} \pi^{2}}{4 w^{2}}\right)\left(k^{2} \beta^{2}-\frac{n^{2} \pi^{2}}{4 w^{2}}\right)} \sum_{S=1}^{\infty} \frac{J_{1}\left(j_{1, s}^{\prime}\right)}{j_{1, s}^{\prime 2} J_{1}^{\prime \prime}\left(j_{1, s}^{\prime}\right)}
\end{array}\right. \\
& \left\langle\left\langle Y_{1}^{(n)}(z)-\frac{Y_{4}^{(n)}(z)}{k^{2} \beta^{2} a^{2}}\right\rangle\right\rangle_{m}=\left\{\begin{array}{l}
\frac{\pi w}{j a\left(\frac{m^{2} \pi^{2}}{4 w^{2}}-k^{2} \beta^{2}\right)}-\frac{\pi k \beta\left[1-(-1)^{m} e^{-j 2 k \beta w}\right]}{a\left(k^{2} \beta^{2}-\frac{m^{2} \pi^{2}}{4 w^{2}}\right)^{2}} \\
\hline w^{2} \beta^{2} a\left(k^{2} \beta^{2}-\frac{1, s^{2}}{a^{2}}-\frac{m^{2} \pi^{2}}{4 w^{2}}\right) \\
k^{2}
\end{array}\right. \\
& \begin{array}{l}
+\sum_{s=1}^{\infty} \frac{2 \pi w}{j a\left(\frac{m^{2} \pi^{2}}{4 w^{2}}-k^{2} \beta^{2}+\frac{j_{1, s}^{2}}{a^{2}}\right)}-\sum_{s=1}^{\infty} \frac{2 \pi \sqrt{k^{2} \beta^{2}-\frac{j_{1, s}^{2}}{a^{2}}}\left[1-(-1)^{m} e^{-j 2} \sqrt{k^{2} \beta^{2}-\frac{j_{1, s}^{2}}{a^{2}} w}\right]}{a\left(k^{2} \beta^{2}-\frac{j_{1, s}^{2}}{a^{2}}-\frac{m^{2} \pi^{2}}{4 w^{2}}\right)^{2}} \\
+\sum_{s=1}^{\infty} \frac{8 \pi^{3} m^{2} w^{3}}{j k^{2} \beta^{2} a^{3}\left(m^{2} \pi^{2}-4 w^{2} k^{2} \beta^{2}\right)\left[m^{2} \pi^{2}-4 w^{2}\left(k^{2} \beta^{2}-\frac{j_{1, s}{ }^{\prime 2}}{a^{2}}\right)\right]} \frac{J_{1}\left(j_{1, s}\right)}{J_{1}^{\prime \prime}\left(j_{1, s}{ }^{\prime}\right)}
\end{array} \\
& -\frac{2 \pi k \beta\left[1-(-1)^{m} e^{-j 2 k \beta w}\right]}{a\left(k^{2} \beta^{2}-\frac{m^{2} \pi^{2}}{4 w^{2}}\right)^{2}} \sum_{s=1}^{\infty} \frac{J_{1}\left(j_{1, s}{ }^{\prime}\right)}{j_{1, s}{ }^{\prime 2} J_{1}{ }^{\prime}\left(j_{1, s}{ }^{\prime}\right)} \\
& +\sum_{s=1}^{\infty} \frac{2 \pi\left(k^{2} \beta^{2}-\frac{j_{1, s}{ }^{\prime 2}}{a^{2}}\right)^{\frac{3}{2}}\left[1-(-1)^{m} e^{-j 2} \sqrt{k^{2} \beta^{2}-\frac{j_{1, s}{ }^{\prime 2}}{a^{2}} w}\right]}{k^{2} \beta^{2} a\left(k^{2} \beta^{2}-\frac{j_{1, s}{ }^{\prime 2}}{a^{2}}-\frac{m^{2} \pi^{2}}{4 w^{2}}\right)^{2}} \frac{J_{1}\left(j_{1, s}{ }^{\prime}\right)}{j_{1, s}{ }^{\prime 2} J_{1}{ }^{\prime \prime}\left(j_{1, s}{ }^{\prime}\right)}, \quad \text { for } n=m,
\end{aligned}
$$




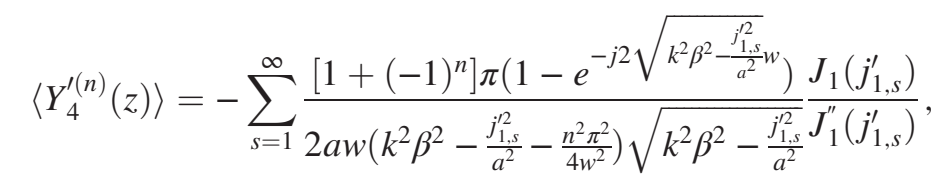

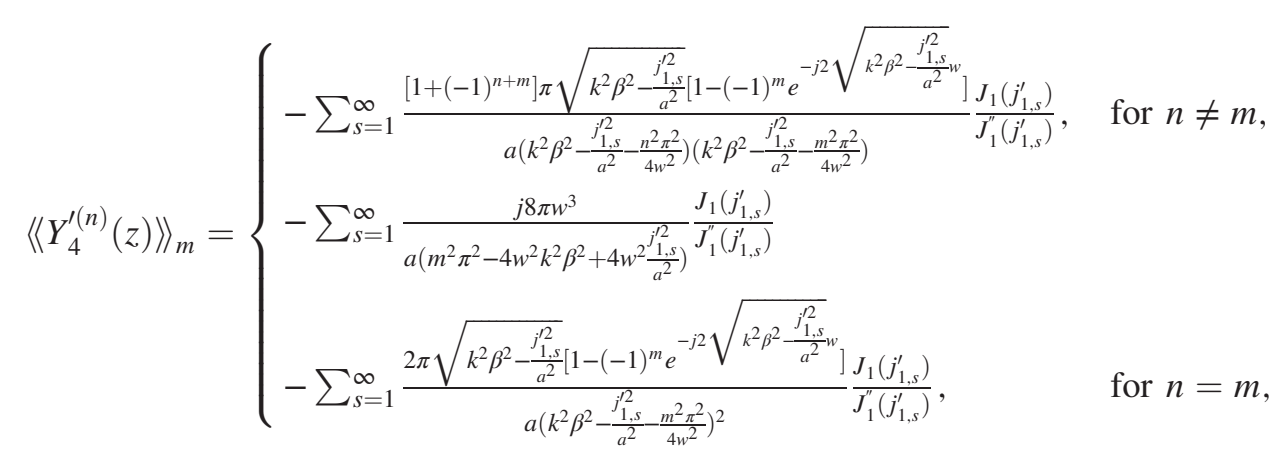

$$
\begin{aligned}
& \left\langle Y_{2}(z)-\frac{Y_{3}(z)}{k^{2} \beta^{2} a^{2}}\right\rangle=\sum_{s=1}^{\infty}\left[\frac{2 \pi a}{w b_{1, s}^{2}}+\frac{\pi a^{2}\left(e^{-j \frac{2 b_{1, s} a}{a}}-1\right)}{j w^{2} b_{1, s}^{3}}-\frac{2 \pi J_{1}\left(j_{1, s}^{\prime}\right)}{k^{2} \beta^{2} a w J_{1}^{\prime \prime}\left(j_{1, s}^{\prime}\right) j_{1, s}^{\prime 2}}-\frac{\pi J_{1}\left(j_{1, s}^{\prime}\right)\left(e^{-j \frac{2 b_{1, s}^{\prime}}{a} w}-1\right)}{j b_{1, s}^{\prime} k^{2} \beta^{2} w^{2} J_{1}^{\prime \prime}\left(j_{1, s}^{\prime}\right) j_{1, s}^{\prime 2}}\right] \\
& \left\langle\left\langle Y_{2}(z)-\frac{Y_{3}(z)}{k^{2} \beta^{2} a^{2}}\right\rangle\right\rangle_{m}=\frac{j \pi\left[1+(-1)^{m}\right]\left(1-e^{-j 2 k \beta w}\right)}{2 k \beta w a\left(k^{2} \beta^{2}-\frac{m^{2} \pi^{2}}{4 w^{2}}\right)} \\
& +\frac{\pi j}{w a} \sum_{s=1}^{\infty} \frac{\left[1+(-1)^{m}\right]\left[1-e^{-j 2 \sqrt{k^{2} \beta^{2}-\frac{j_{1, s}^{2}}{a^{2}} w}}\right]}{\left(k^{2} \beta^{2}-\frac{j_{1, s}^{2}}{a^{2}}-\frac{m^{2} \pi^{2}}{4 w^{2}}\right) \sqrt{k^{2} \beta^{2}-\frac{j_{1, s}^{2}}{a^{2}}}} \\
& +\frac{\pi j\left[1+(-1)^{m}\right]\left(1-e^{-j 2 k \beta w}\right)}{a k \beta w\left(k^{2} \beta^{2}-\frac{m^{2} \pi^{2}}{4 w^{2}}\right)} \sum_{s=1}^{\infty} \frac{J_{1}\left(j_{1, s}^{\prime}\right)}{j_{1, s}^{\prime 2} J_{1}^{\prime \prime}\left(j_{1, s}^{\prime}\right)}
\end{aligned}
$$

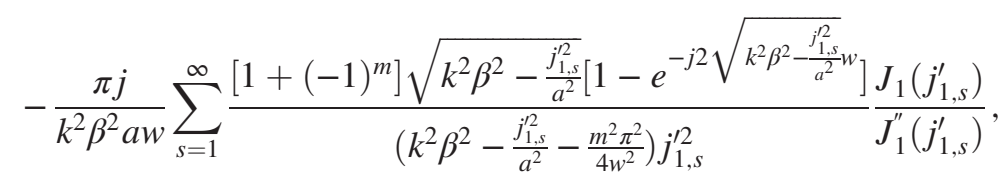

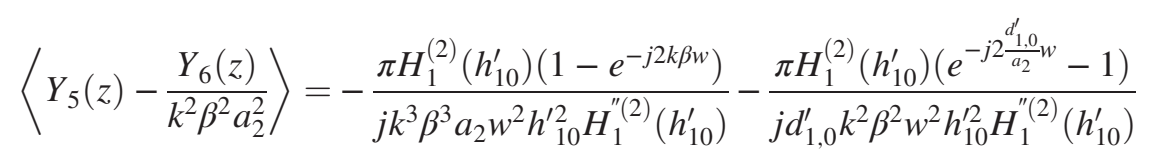

$$
\begin{aligned}
& -\frac{\pi}{k^{2} \beta^{2} a_{2} w}-\frac{\pi\left(e^{-j 2 k \beta w}-1\right)}{j 2 k^{3} \beta^{3} a_{2} w^{2}} \\
& -\frac{2}{\pi w a_{2}} \int_{0}^{\infty} d \zeta \frac{1}{\zeta\left(k^{2} \beta^{2}+\frac{\zeta}{a_{2}^{2}}\right) H_{1}^{(1)}\left(e^{j \frac{\pi}{2}} \sqrt{\zeta}\right) H_{1}^{(2)}\left(e^{j \frac{\pi}{2}} \sqrt{\zeta}\right)} \\
& -\frac{1}{j \pi w^{2} a_{2}} \int_{0}^{\infty} d \zeta \frac{\left(e^{-j 2 \sqrt{k^{2} \beta^{2}+\frac{\zeta}{a_{2}^{2}} w}}-1\right)}{\zeta\left(k^{2} \beta^{2}+\frac{\zeta}{a_{2}^{2}} H_{1}^{\frac{3}{2}} H_{1}^{(1)}\left(e^{j \frac{\pi}{2}} \sqrt{\zeta}\right) H_{1}^{(2)}\left(e^{j \frac{\pi}{2}} \sqrt{\zeta}\right)\right.} \\
& +\frac{1}{j k^{3} \beta^{3} a_{2} w^{2} \pi} \int_{0}^{\infty} d \zeta \frac{\left(1-e^{-j 2 k \beta w}\right)}{\zeta^{2} H_{1}^{\prime(1)}\left(e^{j \frac{\pi}{2}} \sqrt{\zeta}\right) H_{1}^{\prime(2)}\left(e^{j \frac{\pi}{2}} \sqrt{\zeta}\right)} \\
& -\frac{1}{j k^{2} \beta^{2} a_{2} w^{2} \pi} \int_{0}^{\infty} d \zeta \frac{\left(1-e^{-j 2} \sqrt{k^{2} \beta^{2}+\frac{\zeta}{a_{2}^{2}} w}\right)}{\sqrt{k^{2} \beta^{2}+\frac{\zeta}{a_{2}^{2}}} \zeta^{2} H_{1}^{(1)}\left(e^{j \frac{\pi}{2}} \sqrt{\zeta}\right) H_{1}^{\prime(2)}\left(e^{j \frac{\pi}{2}} \sqrt{\zeta}\right)}
\end{aligned}
$$


SHOBUDA, CHIN, AND TAKATA

Phys. Rev. ST Accel. Beams 17, 091001 (2014)

$$
\begin{aligned}
& \left\langle\left\langle Y_{5}(z)-\frac{Y_{6}(z)}{k^{2} \beta^{2} a_{2}^{2}}\right\rangle\right\rangle_{m}=-\frac{\pi j\left[1+(-1)^{m}\right]\left(1-e^{-j 2 k \beta w}\right)}{2 k \beta w a_{2}\left(k^{2} \beta^{2}-\frac{m^{2} \pi^{2}}{4 w^{2}}\right)} \\
& -\frac{j\left[1+(-1)^{m}\right]}{w \pi a_{2}} \int_{0}^{\infty} d \zeta \frac{\left(1-e^{-j 2 \sqrt{\frac{\zeta}{a_{2}^{2}}+k^{2} \beta^{2}} w}\right)}{\zeta\left(\frac{\zeta}{a_{2}^{2}}+k^{2} \beta^{2}-\frac{m^{2} \pi^{2}}{4 w^{2}}\right) \sqrt{\frac{\zeta}{a_{2}^{2}}+k^{2} \beta^{2}} H_{1}^{(1)}\left(e^{\frac{\pi}{2} j} \sqrt{\zeta}\right) H_{1}^{(2)}\left(e^{\frac{\pi}{2} j} \sqrt{\zeta}\right)} \\
& +\frac{\pi j\left[1+(-1)^{m}\right]}{k \beta a_{2} w} \frac{H_{1}^{(2)}\left(h_{1,0}^{\prime}\right)}{H_{1}^{\prime \prime 2}\left(h_{1,0}^{\prime}\right)} \frac{\left(1-e^{-j 2 k \beta w}\right)}{\left(k^{2} \beta^{2}-\frac{m^{2} \pi^{2}}{4 w^{2}}\right) h_{1,0}^{\prime 2}} \\
& -\frac{\pi j\left[1+(-1)^{m}\right]}{k^{2} \beta^{2} a_{2} w} \frac{H_{1}^{(2)}\left(h_{1,0}^{\prime}\right)}{H_{1}^{\prime \prime}(2)\left(h_{1,0}^{\prime}\right)} \frac{\sqrt{k^{2} \beta^{2}-\frac{h_{1,0}^{\prime 2}}{a_{2}^{2}}}\left(1-e^{\left.-j 2 \sqrt{k^{2} \beta^{2}-\frac{h_{1,0}^{\prime 2}}{a_{2}^{2}} w}\right)}\right.}{\left(k^{2} \beta^{2}-\frac{h_{1,0}^{\prime 2}}{a_{2}^{2}}-\frac{m^{2} \pi^{2}}{4 w^{2}}\right) h_{1,0}^{\prime 2}} \\
& -\frac{j\left[1+(-1)^{m}\right]\left(1-e^{-j 2 k \beta w}\right)}{k \beta \pi a_{2} w\left(k^{2} \beta^{2}-\frac{m^{2} \pi^{2}}{4 w^{2}}\right)} \int_{0}^{\infty} d \zeta \frac{1}{\zeta^{2} H_{1}^{\prime(1)}\left(e^{\frac{\pi}{2} j} \sqrt{\zeta}\right) H_{1}^{\prime(2)}\left(e^{\frac{\pi}{2} j} \sqrt{\zeta}\right)} \\
& +\frac{j\left[1+(-1)^{m}\right]}{k^{2} \beta^{2} \pi a_{2} w} \int_{0}^{\infty} d \zeta \frac{\sqrt{\frac{\zeta}{a_{2}^{2}}+k^{2} \beta^{2}}\left(1-e^{-j 2 \sqrt{\frac{\zeta}{a_{2}^{2}} k^{2} \beta^{2} w}}\right)}{\zeta^{2}\left(\frac{\zeta}{a_{2}^{2}}+k^{2} \beta^{2}-\frac{m^{2} \pi^{2}}{4 w^{2}}\right) H_{1}^{\prime(1)}\left(e^{\frac{\pi}{2} j} \sqrt{\zeta}\right) H_{1}^{(2)}\left(e^{\frac{\pi}{2} j} \sqrt{\zeta}\right)},
\end{aligned}
$$

$$
\begin{aligned}
& \left\langle Y_{7}^{(n)}(z)-\frac{Y_{8}^{(n)}(z)}{k^{2} \beta^{2} a_{2}^{2}}\right\rangle=\frac{\pi\left[1+(-1)^{n}\right]}{4 k \beta a_{2} w} \frac{\left(1-e^{-j 2 k \beta w}\right)}{\left(k^{2} \beta^{2}-\frac{n^{2} \pi^{2}}{4 w^{2}}\right)} \\
& +\frac{\left[1+(-1)^{n}\right]}{2 \pi a_{2} w} \int_{0}^{\infty} d \zeta \frac{\left(1-e^{-j 2 \sqrt{\frac{\zeta}{a_{2}^{2}}+k^{2} \beta^{2}} w}\right)}{\zeta \sqrt{\frac{\zeta}{a_{2}^{2}}+k^{2} \beta^{2}}\left(\frac{\zeta}{a_{2}^{2}}+k^{2} \beta^{2}-\frac{n^{2} \pi^{2}}{4 w^{2}}\right) H_{1}^{(1)}\left(e^{\frac{\pi}{2} j} \sqrt{\zeta}\right) H_{1}^{(2)}\left(e^{\frac{\pi}{2} j} \sqrt{\zeta}\right)} \\
& -\frac{\left.\pi\left[1+(-1)^{n}\right)\right]\left(1-e^{-j 2 k \beta w}\right)}{2 k \beta w a_{2}\left(k^{2} \beta^{2}-\frac{n^{2} \pi^{2}}{4 w^{2}} h_{1,0}^{\prime 2}\right.} \frac{H_{1}^{(2)}\left(h_{1,0}^{\prime}\right)}{H_{1}^{\prime \prime(2)}\left(h_{1,0}^{\prime}\right)} \\
& +\frac{\pi\left[1+(-1)^{n}\right] \sqrt{k^{2} \beta^{2}-\frac{h_{1,0}^{\prime 2}}{a_{2}^{2}}}\left(1-e^{-j 2 \sqrt{k^{2} \beta^{2}-\frac{h_{10}^{\prime 2}}{a_{2}^{2}}} w}\right)}{2 w k^{2} \beta^{2} a_{2}\left(k^{2} \beta^{2}-\frac{h_{1,0}^{2}}{a_{2}^{2}}-\frac{n^{2} \pi^{2}}{4 w^{2}}\right) h_{1,0}^{\prime 2}} \frac{H_{1}^{(2)}\left(h_{1,0}^{\prime}\right)}{H_{1}^{\prime \prime}(2)\left(h_{1,0}^{\prime}\right)} \\
& +\frac{\left[1+(-1)^{n}\right]\left(1-e^{-j 2 k \beta w}\right)}{2 k \beta \pi w a_{2}\left(k^{2} \beta^{2}-\frac{n^{2} \pi^{2}}{4 w^{2}}\right)} \int_{0}^{\infty} d \zeta \frac{1}{\zeta^{2} H_{1}^{\prime(1)}\left(e^{\frac{\pi}{2} j} \sqrt{\zeta}\right) H_{1}^{\prime(2)}\left(e^{\frac{\pi}{2} j} \sqrt{\zeta}\right)} \\
& -\frac{\left[1+(-1)^{n}\right]}{2 \pi w k^{2} \beta^{2} a_{2}} \int_{0}^{\infty} d \zeta \frac{\sqrt{\frac{\zeta}{a_{2}^{2}}+k^{2} \beta^{2}}\left(1-e^{-j 2 \sqrt{\frac{\zeta}{a_{2}^{2}} k^{2} \beta^{2} w}}\right)}{\zeta^{2}\left(\frac{\zeta}{a_{2}^{2}}+k^{2} \beta^{2}-\frac{n^{2} \pi^{2}}{4 w^{2}}\right) H_{1}^{\prime(1)}\left(e^{\frac{\pi}{2} j} \sqrt{\zeta}\right) H_{1}^{\prime(2)}\left(e^{\frac{\pi}{2} j} \sqrt{\zeta}\right)},
\end{aligned}
$$

091001-36 


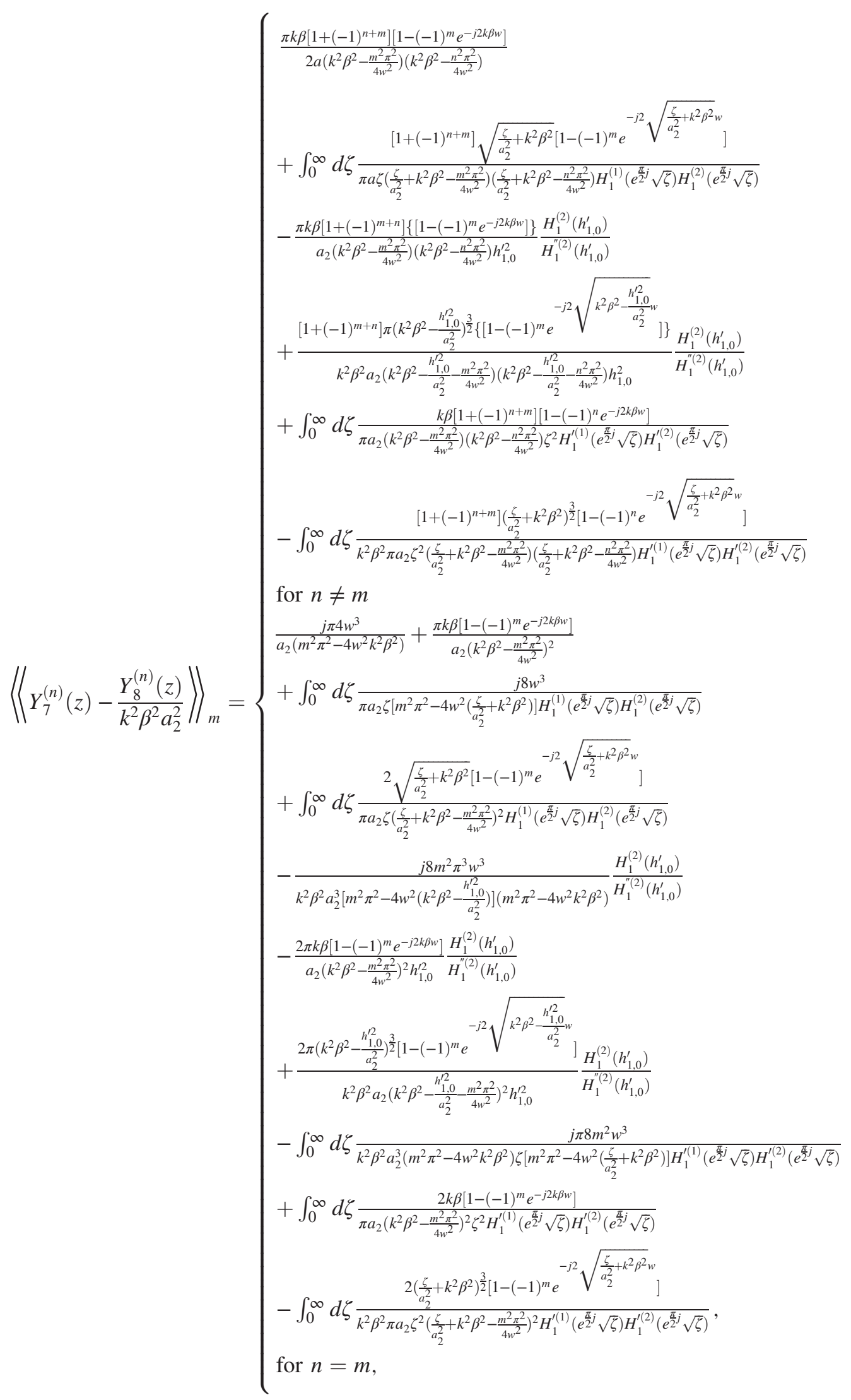




$$
\begin{aligned}
\left\langle Y_{9}^{(n)}(z)\right\rangle= & -\frac{\pi\left[1+(-1)^{n}\right]\left(1-e^{\left.-j 2 \sqrt{k^{2} \beta^{2}-\frac{h_{1,0}^{\prime 2}}{a_{2}^{2}} w}\right)}\right.}{2 w a_{2}\left(k^{2} \beta^{2}-\frac{h_{1,0}^{\prime 2}}{a_{2}^{2}}-\frac{n^{2} \pi^{2}}{4 w^{2}}\right) \sqrt{k^{2} \beta^{2}-\frac{h_{1,2}^{\prime 2}}{a_{2}^{2}}} \frac{H_{1}^{(2)}\left(h_{1,0}^{\prime}\right)}{H_{1}^{\prime \prime(2)}\left(h_{1,0}^{\prime}\right)}} \\
& -\frac{\left[1+(-1)^{n}\right]}{2 w \pi a_{2}} \int_{0}^{\infty} d \zeta \frac{\left(1-e^{\left.-j 2 \sqrt{\frac{\zeta}{a_{2}^{2}}+k^{2} \beta^{2} w}\right)}\right.}{\zeta \sqrt{\frac{\zeta}{a_{2}^{2}}+k^{2} \beta^{2}}\left(\frac{\zeta}{a_{2}^{2}}+k^{2} \beta^{2}-\frac{n^{2} \pi^{2}}{4 w^{2}}\right) H_{1}^{\prime(1)}\left(e^{\frac{\pi}{2} j} \sqrt{\zeta}\right) H_{1}^{\prime(2)}\left(e^{\frac{\pi}{2} j} \sqrt{\zeta}\right)},
\end{aligned}
$$

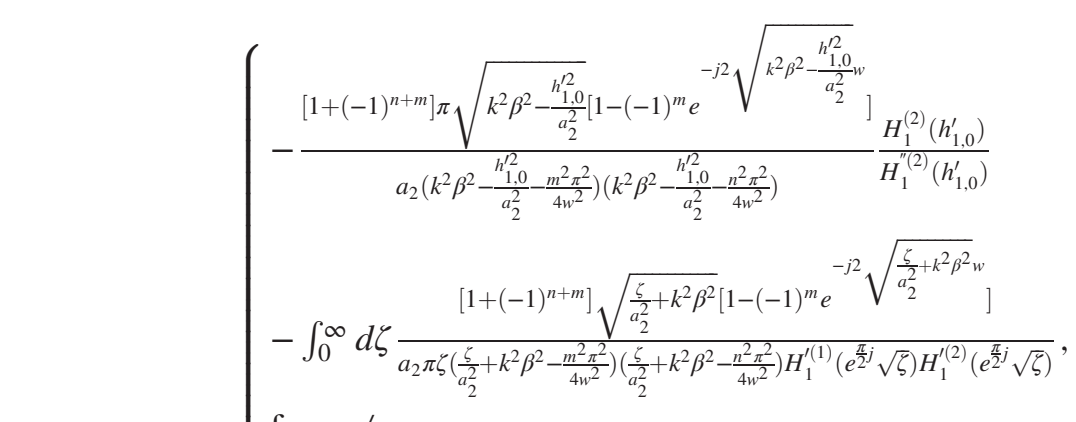

for $n \neq m$,

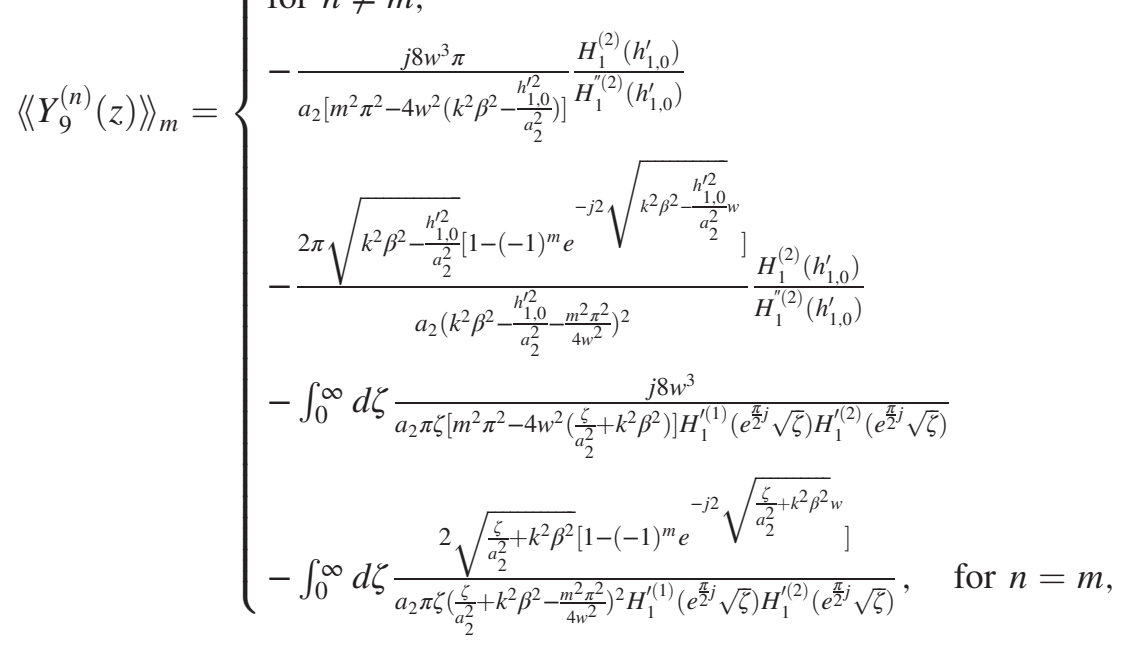

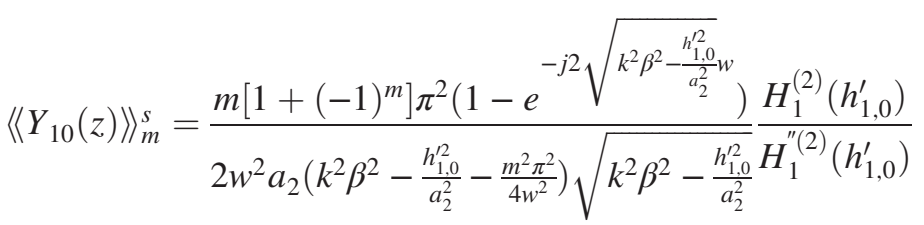

$$
\begin{aligned}
& +\frac{m\left[1+(-1)^{m}\right]}{2 w^{2} a_{2}} \int_{0}^{\infty} d \zeta \frac{\left(1-e^{\left.-j 2 \sqrt{\frac{\zeta}{a_{2}^{2}+k^{2} \beta^{2}} w}\right)}\right.}{\zeta \sqrt{\frac{\zeta}{a_{2}^{2}}+k^{2} \beta^{2}}\left(\frac{\zeta}{a_{2}^{2}}+k^{2} \beta^{2}-\frac{m^{2} \pi^{2}}{4 w^{2}}\right) H_{1}^{(1)}\left(e^{\frac{\pi}{2} j} \sqrt{\zeta}\right) H_{1}^{\prime(2)}\left(e^{\frac{\pi}{2} j} \sqrt{\zeta}\right)},
\end{aligned}
$$




$$
\begin{aligned}
& \left\{\begin{array}{l}
\frac{j\left[1+(-1)^{n+m}\right] m \pi^{2} \sqrt{k^{2} \beta^{2}-\frac{h_{1,0}^{\prime 2}}{a_{2}^{2}}}\left[1-(-1)^{m} e^{-j 2} \sqrt{k^{2} \beta^{2}-\frac{h_{1,0}^{\prime 2}}{a_{2}^{2}} w}\right]}{2 w a_{2}\left(k^{2} \beta^{2}-\frac{h_{1,0}^{\prime 2}}{a_{2}^{2}}-\frac{m^{2} \pi^{2}}{4 w^{2}}\right)\left(k^{2} \beta^{2}-\frac{h_{1,0}^{\prime 2}}{a_{2}^{2}}-\frac{n^{2} \pi^{2}}{4 w^{2}}\right)} \frac{H_{1}^{(2)}\left(h_{1,0}^{\prime}\right)}{H_{1}^{\prime(2)}\left(h_{1,0}^{\prime}\right)} \\
+\int_{0}^{\infty} d \zeta \frac{j m\left[1+(-1)^{n+m}\right] \sqrt{\frac{\zeta}{a_{2}^{2}}+k^{2} \beta^{2}}\left[1-(-1)^{m} e^{-j 2} \sqrt{\frac{\zeta}{a_{2}^{2}+k^{2} \beta^{2}} w}\right]}{2 w a_{2} \zeta\left(\frac{\zeta}{a_{2}^{2}}+k^{2} \beta^{2}-\frac{m^{2} \pi^{2}}{4 w^{2}}\right)\left(\frac{\zeta}{a_{2}^{2}}+k^{2} \beta^{2}-\frac{n^{2} \pi^{2}}{4 w^{2}}\right) H_{1}^{\prime(1)}\left(e^{\frac{\pi}{2} j} \sqrt{\zeta}\right) H_{1}^{\prime(2)}\left(e^{\frac{\pi}{2} j} \sqrt{\zeta}\right)}
\end{array}\right.
\end{aligned}
$$

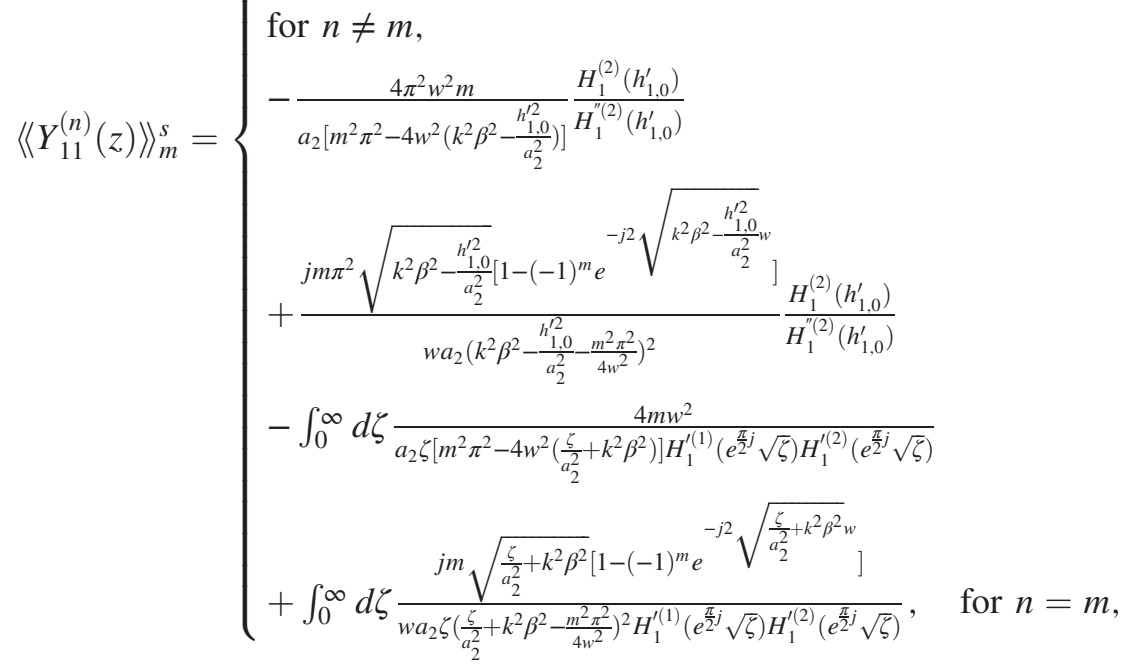

$$
\left\{\begin{array}{l}
\frac{j m\left[1+(-1)^{n+m}\right] \pi^{2}\left[1-(-1)^{m} e^{-j 2} \sqrt{k^{2} \beta^{2}-\frac{h_{1,0}^{\prime 2}}{a_{2}^{2}}}\right]}{2 w a_{2}^{3}\left(k^{2} \beta^{2}-\frac{h_{1,0}^{\prime 2}}{a_{2}^{2}}-\frac{m^{2} \pi^{2}}{4 w^{2}}\right)\left(k^{2} \beta^{2}-\frac{h_{1,0}^{\prime 2}}{a_{2}^{2}}-\frac{n^{2} \pi^{2}}{4 w^{2}}\right) \sqrt{k^{2} \beta^{2}-\frac{h_{1,0}^{\prime 2}}{a_{2}^{2}}} \frac{h_{1,0}^{\prime 2}}{H_{1}^{(2)}\left(h_{1,0}^{\prime}\right)}} \\
-\int_{0}^{\infty} d \zeta \frac{j m\left[1+(-1)^{n+m}\right]\left[1-(-1)^{m} e\right.}{2 w a_{2}^{3} \sqrt{\frac{\zeta}{a_{2}^{2}}+k^{2} \beta^{2}}\left(\frac{\zeta}{a_{2}^{2}}+k^{2} \beta^{2}-\frac{m^{2} \pi^{2}}{4 w^{2}}\right)\left(\frac{\zeta}{a_{2}^{2}}+k^{2} \beta^{2}-\frac{n^{2} \pi^{2}}{4 w^{2}}\right) H_{1}^{\prime(1)}\left(e^{\frac{\zeta}{2} j} \sqrt{\zeta}\right) H_{1}^{\prime(2)}\left(e^{\frac{\pi}{2}} j \sqrt{\zeta}\right)},
\end{array}\right.
$$

for $n \neq m$,

$$
\begin{aligned}
& \left.\left\langle Y_{12}^{(n)}(z)\right\rangle\right\rangle_{m}^{S}=\left\{\begin{array}{l}
\frac{4 w^{2}\left(m^{2} \pi^{2}-4 k^{2} \beta^{2} w^{2}\right)}{m a_{2}\left[m^{2} \pi^{2}-4 w^{2}\left(k^{2} \beta^{2}-\frac{h_{1,0}^{\prime 2}}{a_{2}^{2}}\right)\right]} \frac{H_{1}^{(2)}\left(h_{1,0}^{\prime}\right)}{H_{1}^{\prime(2)}\left(h_{1,0}^{\prime}\right)} \\
+\frac{j m \pi^{2}\left[1-(-1)^{m} e^{-j 2} \sqrt{k^{2} \beta^{2}-\frac{h_{1,0}^{\prime 2}}{a_{2}^{2}}}\right.}{w a_{2}^{3}\left(k^{2} \beta^{2}-\frac{h_{1,0}^{\prime 2}}{a_{2}^{2}}-\frac{m^{2} \pi^{2}}{4 w^{2}}\right)^{2} \sqrt{k^{2} \beta^{2}-\frac{h_{1,0}^{\prime 2}}{a_{2}^{2}}} \frac{h_{1,0}^{\prime 2} H_{1}^{(2)}\left(h_{1,0}^{\prime}\right)}{H_{1}^{\prime \prime(2)}\left(h_{1,0}^{\prime}\right)}}
\end{array}\right. \\
& +\int_{0}^{\infty} d \zeta \frac{4 w^{2}\left(m^{2} \pi^{2}-4 k^{2} \beta^{2} w^{2}\right)}{m \pi^{2} a_{2} \zeta\left[m^{2} \pi^{2}-4 w^{2}\left(\frac{\zeta}{a_{2}^{2}}+k^{2} \beta^{2}\right)\right] H_{1}^{\prime(1)}\left(e^{\frac{\hbar}{2} j} \sqrt{\zeta}\right) H_{1}^{(2)}\left(e^{\frac{\pi}{2} j} \sqrt{\zeta}\right)} \\
& -\int_{0}^{\infty} d \zeta \frac{j m\left[1-(-1)^{m} e^{\left.-j 2 \sqrt{\frac{\zeta}{a_{2}^{2}+k^{2} \beta^{2}} w}\right]}\right.}{w a_{2}^{3} \sqrt{\frac{\zeta}{a_{2}^{2}}+k^{2} \beta^{2}}\left(\frac{\zeta}{a_{2}^{2}}+k^{2} \beta^{2}-\frac{m^{2} \pi^{2}}{4 w^{2}}\right)^{2} H_{1}^{\prime(1)}\left(e^{\frac{\pi}{2} j} \sqrt{\zeta}\right) H_{1}^{\prime(2)}\left(e^{\frac{\pi}{2} j} \sqrt{\zeta}\right)},
\end{aligned}
$$

for $n=m$, 
where $\quad b_{1, s}=\sqrt{k^{2} \beta^{2} a^{2}-j_{1, s}^{2}}=-j \sqrt{j_{1, s}^{2}-k^{2} \beta^{2} a^{2}}$, $b_{1, s}^{\prime}=\sqrt{k^{2} \beta^{2} a^{2}-j_{1, s}^{\prime 2}}=-j \sqrt{j_{1, s}^{\prime 2}-k^{2} \beta^{2} a^{2}}$ and $d_{1,0}^{\prime}=$ $\sqrt{k^{2} \beta^{2} a^{2}-h_{1,0}^{\prime 2}}$. It is particularly notable that there are no singular points except $\zeta=0$ in the integrands of $\zeta$ integrations. In some of the above equations, it looks as if there are singular points in the integrands between $\zeta=0$ and infinity. However, the integrands were shown to be regular at seemingly singular points by simplifying the relevant terms and taking a limit.

\section{APPENDIX E: LONGITUDINAL IMPEDANCES OF THE CERAMIC CHAMBERS WITH THE THIN TIN COATING, COVERED WITH PERFECTLY CONDUCTIVE WALLS}

In this section, let us derive the formula of longitudinal impedances of the ceramic chambers with translation symmetry, where the inner surface is coated with thin TiN and the outer surface is surrounded by a perfectly conductive wall. Following Refs. [12] and [19], field matching technique is applied in the derivation.

When a beam with the current density $j_{z}=$ $\beta c[1-\Theta(\rho-\sigma)] e^{-j k z} /\left(\pi \sigma^{2}\right)$ passes through the chamber, $E_{z}, H_{\theta}$ in vacuum are expressed as

$$
\begin{gathered}
E_{z}=\frac{j k}{\gamma^{2}} \frac{c Z_{0}}{\pi \sigma^{2}}\left(\frac{1}{\bar{k}^{2}}-\frac{\sigma I_{0}(\bar{k} \rho) K_{1}(\bar{k} \sigma)}{\bar{k}}\right)+\overline{\mathcal{A}}_{0}(k) I_{0}(\bar{k} \rho), \\
H_{\theta}=\frac{\beta c}{\pi \sigma} K_{1}(\bar{k} \sigma) I_{1}(\bar{k} \rho)+\frac{j \beta \gamma}{Z_{0}} \overline{\mathcal{A}}_{0}(k) I_{1}(\bar{k} \rho)
\end{gathered}
$$

for $\rho<\sigma$, and

$$
E_{z}=\frac{j c Z_{0}}{\pi \sigma \gamma} I_{1}(\bar{k} \sigma) K_{0}(\bar{k} \rho)+\overline{\mathcal{A}}_{0}(k) I_{0}(\bar{k} \rho),
$$

$$
H_{\theta}=\frac{\beta c}{\pi \sigma} I_{1}(\bar{k} \sigma) K_{1}(\bar{k} \rho)+\frac{j \beta \gamma}{Z_{0}} \overline{\mathcal{A}}_{0}(k) I_{1}(\bar{k} \rho),
$$

for $\sigma<\rho<a$, where $\overline{\mathcal{A}}_{0}(k)$ is an expansion coefficient, $\sigma$ is the radius of the beam and $\Theta(x)$ is the step function.

Inside the thin TiN (for $a<\rho<a+t$ ) with conductivity $\sigma_{\text {TiN }}$, the fields are described as

$$
\begin{gathered}
E_{z} \simeq \bar{B}_{0} I_{0}\left(\kappa_{\mathrm{TiN}} \rho\right)+\bar{C}_{0} K_{0}\left(\kappa_{\mathrm{TiN}} \rho\right), \\
H_{\theta} \simeq \frac{\kappa_{\mathrm{TiN}}}{j k \beta Z_{0}}\left[\bar{B}_{0} I_{1}\left(\kappa_{\mathrm{TiN}} \rho\right)-\bar{C}_{0} K_{1}\left(\kappa_{\mathrm{TiN}} \rho\right)\right],
\end{gathered}
$$

where $\kappa_{\mathrm{TiN}}$ is given by Eq. (39) in the text. Here $\bar{B}_{0}$ and $\bar{C}_{0}$ are expansion coefficients.

Finally, inside the ceramic (for $a+t<\rho<a_{2}$ ) with relative dielectric constant $\epsilon^{\prime}$, they are expanded as

$$
\begin{gathered}
E_{z}=\bar{D}_{0} I_{0}\left(\mu^{k} \rho\right)+\bar{E}_{0} K_{0}\left(\mu^{k} \rho\right), \\
H_{\theta}=-\frac{k \beta \epsilon^{\prime}}{j \mu^{k} Z_{0}}\left[\bar{D}_{0} I_{1}\left(\mu^{k} \rho\right)-\bar{E}_{0} K_{1}\left(\mu^{k} \rho\right)\right],
\end{gathered}
$$

by using the expansion coefficients $\bar{D}_{0}$ and $\bar{E}_{0}$, where

$$
\mu^{k}=\sqrt{k^{2}-k^{2} \beta^{2} \epsilon^{\prime}} .
$$

For the thin TiN, the fields on $\rho=a$ and those on $\rho=a_{2}$ are related to as

$$
\begin{aligned}
& E_{z}\left(a_{2}\right)=A_{0}\left(a_{2}, a\right) E_{z}(a)+C_{0}\left(a_{2}, a\right) H_{\theta}(a), \\
& H_{\theta}\left(a_{2}\right)=I_{0}\left(a_{2}, a\right) E_{z}(a)+K_{0}\left(a_{2}, a\right) H_{\theta}(a),
\end{aligned}
$$

where the transfer coefficients are approximated as

$$
\begin{aligned}
A_{0}\left(a_{2}, a\right) \simeq & \mu^{k} a\left[I_{0}^{\prime}\left(\mu^{k} a\right) K_{0}\left(\mu^{k} a_{2}\right)-I_{0}\left(\mu^{k} a_{2}\right) K_{0}^{\prime}\left(\mu^{k} a\right)\right] \cosh \kappa_{\mathrm{TiN}} t \\
- & \frac{j Z_{0}\left(\mu^{k}\right)^{2} a \sigma_{\mathrm{TiN}}\left[I_{0}\left(\mu^{k} a_{2}\right) K_{0}\left(\mu^{k} a\right)-I_{0}\left(\mu^{k} a\right) K_{0}\left(\mu^{k} a_{2}\right)\right] \sinh \kappa_{\mathrm{TiN}} t}{k \beta \epsilon^{\prime} \kappa_{\mathrm{TiN}}}, \\
C_{0}\left(a_{2}, a\right) \simeq & \frac{\mu^{k} a\left[I_{0}^{\prime}\left(\mu^{k} a\right) K_{0}\left(\mu^{k} a_{2}\right)-I_{0}\left(\mu^{k} a_{2}\right) K_{0}^{\prime}\left(\mu^{k} a\right)\right] \kappa_{\mathrm{TiN}} \sinh \kappa_{\mathrm{TiN}} t}{\sigma_{\mathrm{TiN}}} \\
& -\frac{j Z_{0}\left(\mu^{k}\right)^{2} a\left[I_{0}\left(\mu^{k} a_{2}\right) K_{0}\left(\mu^{k} a\right)-I_{0}\left(\mu^{k} a\right) K_{0}\left(\mu^{k} a_{2}\right)\right] \cosh \kappa_{\mathrm{TiN}} t}{k \beta \epsilon^{\prime}}, \\
I_{0}\left(a_{2}, a\right) \simeq & \frac{j k \beta a \epsilon^{\prime}\left[I_{0}^{\prime}\left(\mu^{k} a\right) K_{0}^{\prime}\left(\mu^{k} a_{2}\right)-I_{0}^{\prime}\left(\mu^{k} a_{2}\right) K_{0}^{\prime}\left(\mu^{k} a\right)\right] \cosh \kappa_{\mathrm{TiN}} t}{Z_{0}} \\
& +\frac{\mu^{k} a \sigma_{\mathrm{TiN}}\left[I_{0}^{\prime}\left(\mu^{k} a_{2}\right) K_{0}\left(\mu^{k} a\right)-I_{0}\left(\mu^{k} a\right) K_{0}^{\prime}\left(\mu^{k} a_{2}\right)\right] \sinh \kappa_{\mathrm{TiN}} t}{\kappa_{\mathrm{TiN}}},
\end{aligned}
$$




$$
\begin{aligned}
K_{0}\left(a_{2}, a\right) \simeq & \frac{j k \beta a \epsilon^{\prime}\left[I_{0}^{\prime}\left(\mu^{k} a\right) K_{0}^{\prime}\left(\mu^{k} a_{2}\right)-I_{0}^{\prime}\left(\mu^{k} a_{2}\right) K_{0}^{\prime}\left(\mu^{k} a\right)\right] \kappa_{\mathrm{TiN}} \sinh \kappa_{\mathrm{TiN}} t}{\sigma_{\mathrm{TiN}} Z_{0}} \\
& +\mu^{k} a\left[I_{0}^{\prime}\left(\mu^{k} a_{2}\right) K_{0}\left(\mu^{k} a\right)-I_{0}\left(\mu^{k} a\right) K_{0}^{\prime}\left(\mu^{k} a_{2}\right)\right] \cosh \kappa_{\mathrm{TiN}} t
\end{aligned}
$$

where the prime in $I_{n}(z)$ and $J_{n}(z)$ means the differential by their argument $z$. It is noticeable that Eqs. (E12)-(E15) are identical to Eqs. (C21)-(C24) in Appendix C 1, when we replace $\mu_{m}^{e}$ in Eqs. (C21)-(C24) with $\mu^{k}$.

The coefficient $\overline{\mathcal{A}}_{0}(k)$ is obtained by combining Eqs. (E3), (E4) and (E10) with the condition $E_{z}\left(a_{2}\right)=0$, and solving the equations. In order to subtract the contribution due to space charge impedance from the final expression of the impedance of the ceramic chamber, the coefficient $\overline{\mathcal{A}}_{0}^{\text {perf }}(k)$, which is obtained by using the assumption that the perfectly conductive walls exist on $\rho=a$, is calculated in advance, and it is given by

$$
\overline{\mathcal{A}}_{0}^{\text {perf }}(k)=-\frac{j c Z_{0}}{\pi \sigma \gamma I_{0}(\bar{k} a)} I_{1}(\bar{k} \sigma) K_{0}(\bar{k} a) .
$$

Finally, a rigorous expression of the longitudinal impedance is obtained as

$$
Z_{L}=Z_{L, s p}+Z_{L, \text { ceramic }}
$$

where

$$
Z_{L, s p}=-\frac{j Z_{0}}{\beta \pi k \sigma^{2}}\left(1-2 I_{1}(\bar{k} \sigma) K_{1}(\bar{k} \sigma)-\frac{2 I_{1}^{2}(\bar{k} \sigma) K_{0}(\bar{k} a)}{I_{0}(\bar{k} a)}\right) \mathcal{L},
$$

$$
Z_{L, \text { ceramic }}=\frac{2 \mathcal{L} C_{0}\left(a_{2}, a\right) I_{1}^{2}(\bar{k} \sigma)}{\pi \sigma^{2} \bar{k}^{2} a I_{0}^{2}(\bar{k} a)\left[A_{0}\left(a_{2}, a\right)+C_{0}\left(a_{2}, a\right) \frac{j \beta \gamma I_{1}(\bar{k} a)}{Z_{0} I_{0}(\bar{k} a)}\right]},
$$

$Z_{L, s p}$ is space charge impedance, $Z_{L, \text { ceramic }}$ is the impedance of ceramic chamber with the TiN coating and $\mathcal{L}$ is length of the chamber.

[1] Y. Shobuda, Y. Irie, and S. Igarashi, Phys. Rev. ST Accel. Beams 12, 032401 (2009).

[2] K. Ohmi, T. Toyama, and C. Ohmori, Phys. Rev. ST Accel. Beams 5, 114402 (2002).

[3] K. Ohmi, T. Toyama, and C. Ohmori, Phys. Rev. ST Accel. Beams 6, 029901(E) (2003).

[4] Y. H. Chin, S. Lee, K. Takata, T. Toyama, Y. Shobuda, and $H$. Tsutsui, Impedance generated by a ceramic chamber with RF shields and TiN coating, in Proceedings of HB2006, Tsukuba, Japan, edited by Y.H.Chin,
H.Yoshikawa, and M.Ikegami (KEK,Tsukuba, Japan, 2006), p. 125.

[5] A. W. Chao, Physics of Collective Beam Instabilities in High Energy Accelerators (Wiley, New York, 1993).

[6] Handbook of Accelerator Physics and Engineering, edited by A. W. Chao and M. Tigner (World Scientific, Singapore, 1999).

[7] http://j-parc.jp/index-e.html.

[8] CST STUDIO SUITE, https://www.cst.com.

[9] Y. H. Chin, KEK Report No. 2005-06, 2005.

[10] Y. Shobuda, Y. H. Chin, and K. Takata, Phys. Rev. ST Accel. Beams 10, 044403 (2007).

[11] Y. Shobuda, Y. H. Chin, and K. Takata, Phys. Rev. ST Accel. Beams 12, 094401 (2009).

[12] B. W. Zotter and S. A. Kheifets, Impedances and Wakes in High-Energy Particle Accelerators (World Scientific, Singapore, 1998).

[13] M. Abramowitz and I. Stegun, Handbook of Mathematical Functions-With Formulas, Graphs, and Mathematical Tables (Dover, New York, 1974).

[14] W. Panofsky and W. Wenzel, Rev. Sci. Instrum. 27, 967 (1956)

[15] N. Biancacci, V. G. Vaccaro, E. Metral, B. Salvant, M. Migliorati, and L. Palumbo, Phys. Rev. ST Accel. Beams 17, 021001 (2014).

[16] H. Tsutsui, Internal Report, (in Japanese) 2001.

[17] S. Lee, Simplified ZL model of the ceramic pipe in RCS, 2003, Internal Report (in Japanese), 2003, http:// www-accps.kek.jp/J-PARC/beam_instability/ index-impedance_budget.html.

[18] Y. Shobuda, Y.H. Chin, K. Ohmi, and T. Toyama, The impedance of the ceramic chamber in J-PARC, in Proceedings of the 21st Particle Accelerator Conference, Knoxville, TN, 2005 (IEEE, Piscataway, NJ, 2005), p. 1898.

[19] E. Metral, B. Zotter, and B. Salvant, Resistive-wall impedance of an infinitely long multi-layer cylindrical beam pipe, in Proceedings of the 22nd Particle Accelerator Conference, PAC-2007, Albuquerque, NM (IEEE, New York, 2007), p. 4216.

[20] N. Mounet and E. Metral, Impedances of an infinitely long and axisymmetric multilayer beam pipe: Matrix formalism and multimode analysis, in Proceedings of the International Particle Accelerator Conference, Kyoto, Japan (ICR, Kyoto, 2010), p. 2039.

[21] J. Bento, F. Caspers, and A. Mostacci, CERN PS/RF/Note 2002-217, 2002.

[22] S. Silver and W. K. Saunders, J. Appl. Phys. 21, 153 (1950).

[23] R. L. Gluckstern and R. Li, Part. Accel. 29, 159 (1990).R. L. Gluckstern and R. Li, Proceeding of the 14th International Conference on High-energy Accelerators (Tsukuba, Japan, 1989), p. 861. 